\title{
A UNIFIED MODE DECOMPOSITION METHOD FOR PHYSICAL FIELDS IN HOMOGENEOUS COSMOLOGY
}

\author{
ZHIRAYR G. AVETISYAN \\ Max Planck Institute for Mathematics in the Sciences, Inselstr. 22, \\ 04103 Leipzig, Germany \\ jirayrag@gmail.com \\ Institut für Theoretische Physik, Universität Leipzig, Brüderstr. 16, \\ 04103 Leipzig, Germany
}

\begin{abstract}
The methods of mode decomposition and Fourier analysis of classical and quantum fields on curved spacetimes previously available mainly for the scalar field on FriedmanRobertson-Walker (FRW) spacetimes are extended to arbitrary vector bundle fields on general spatially homogeneous spacetimes. This is done by developing a rigorous unified framework which incorporates mode decomposition, harmonic analysis and Fourier analysis. The limits of applicability and uniqueness of mode decomposition by separation of the time variable in the field equation are found. It is shown how mode decomposition can be naturally extended to weak solutions of the field equation under some analytical assumptions. It is further shown that these assumptions can always be fulfilled if the vector bundle under consideration is analytic. The propagator of the field equation is explicitly mode decomposed. A short survey on the geometry of the models considered in mathematical cosmology is given and it is concluded that practically all of them can be represented by a semidirect homogeneous vector bundle. Abstract harmonic analytical Fourier transform is introduced in semidirect homogeneous spaces and it is explained how it can be related to the spectral Fourier transform. The general form of invariant bi-distributions on semidirect homogeneous spaces is found in the Fourier space which generalizes earlier results for the homogeneous states of the scalar field on FRW spacetimes.

Keywords: Mode decomposition; propagator; homogeneous cosmology; homogeneous states.
\end{abstract}

Mathematics Subject Classification 2000: 83F05, 81T20, 43A85

\section{Introduction}

As long as mankind is not in possession of a successful and commonly accepted quantum theory of gravitation (and possibly even thereafter), the quantum field theory on curved spacetimes (QFT in CST) is an adequate and consistent theoretical framework for astronomy and cosmology. Vicinities of black holes and the early epoch of the universe are two prominent physical situations where gravity is sufficiently strong so that its influence on the quantum field theory cannot be neglected. At the same time, in these situations the gravity is sufficiently uniform (i.e., its local fluctuations are negligible) to be considered classical and interact- 
ing with matter quantum fields mainly macroscopically. This semiclassical picture is captured by letting quantum fields propagate on a curved spacetime. The back reaction of the matter on gravity is described by the semiclassical Einstein equation, where gravity feels only the expectation values of quantum entities. This is the domain of quantum field theory on curved spacetimes, apart from its intrinsic appeal for the beauty and variety of fundamental problems it poses in mathematical physics. QFT in CST adopts the more modern algebraic quantum field theory setup and, as appropriate to mathematical physics, attempts to be as axiomatic and deductive as possible and mathematically rigorous. The disadvantage of such an approach is the extreme difficulty of producing explicit ready-made results which can be applied on the observational level, and each such result can be considered as a remarkable success. For instance, it was not until 2010 when the authors of [1] obtained the first completely rigorous and at the same time explicit description of cosmological particle creation in states of low energy. This was done under several assumptions which can or cannot be considered realistic in cosmology. Namely, the Klein-Gordon field on FRW spacetimes was chosen, and homogeneity and sufficient regularity was stipulated a priori for the desired state of low energy. When one tries to step a bit beyond these restrictions one immediately faces severe mathematical difficulties along the entire way from the very setup until the final expressions. The reason is that the chain of results used in these constructions has been obtained only under the above mentioned assumptions. The aim of the current work is the extension of some of those mathematical methods to a generality where they can be applied for practically all realistic cosmological situations. To which extent this program has been successful will become clear below.

A primary tool for obtaining explicit constructions are geometric symmetries. After publishing his eminent work on general relativity, Einstein declared he had no hope to see explicit solutions of his equation in the near future. It was the rich symmetry of the FRW spacetimes that allowed Friedmann to find first explicit solutions and thus to dispel the despair of Einstein shortly after his publication. This instant can be considered as the birth of modern mathematical cosmology, which until today remains one of the main appliers of explicit solutions in general relativity. One of the merits of geometric symmetries is the possibility of the separation of variables in the field equation which helps to obtain explicit solutions. The mode decomposition of the solutions of the field equation (also referred to as the Fourier method in PDE, or expansion into harmonic oscillators in physics) was probably first applied in the cosmological context by Parker [2] who performed it on the flat FRW spacetime. The idea of the method is that one tries to separate the time variable in the field equation, and looks for solutions as linear combinations of products $X(\vec{x}) T(t)$ where $X$ depends only on the spatial coordinates and $T$ only on time. What Parker discovered is that this is possible on FRW spacetimes and represents a very handy tool for the analysis of the dynamics. A thorough analytical investigation of the method in the cosmological context was conducted in [3], where an abstract functional analytical eigenfunction expansion was introduced as 
a methodological background, and precise methods were suggested for the mode decomposition of regular solutions on FRW and ultrastatic spacetimes. The theory of the method does not seem to have been developed any further until nowadays. In particular, the following questions remain open. What are the precise limits of applicability of the mode decomposition by means of separation of the time variable? How many different decompositions are possible for the same geometrical setup? When and how can the decomposition be extended to weak (distributional) solutions?

In the first part of this work satisfactory answers will be given to these questions and establish a unified framework for the method. Our geometrical setup will be a finite dimensional vector bundle over an arbitrary globally hyperbolic Lorentzian manifold furnished with a pseudo-Riemannian fiber metric and a fiber metric linear connection. The field equation will then be given by the field operator $D=\square^{\nabla}+m^{\star}(x)$ where $\square^{\nabla}$ is the connection d'Alambert operator acting on the smooth sections, and $m^{\star}(x)$ will be the variable smooth "mass term" (possibly including a coupling to scalar curvature) to which mild assumptions will be imposed. This seems to be the most general setup of a (symmetric) hyperbolic linear field on a curved spacetime, and covers most practical situations in the cosmological context.

The results can be briefly described as follows. Precise geometrical necessary and sufficient conditions are obtained for the mode decomposition of smooth solutions by time separation to be realizable. This mode decomposition is given, as perhaps expected, by the time dependent Fourier transform, and is shown to be basically the only such mode decomposition possible. The decomposition is extended to all distributional solutions in a natural manner given that there exists a choice of modes fulfilling certain regularity conditions. The conditions become fully explicit once one has a Paley-Wiener theorem for the spatial Fourier transform, i.e., a precise description of the Fourier image of the test functions space. This is the situation in FRW spaces. Moreover, it is shown that if the bundle is analytic and the dynamics of the geometry sufficiently rigid (precise definitions are given) then the conditions are satisfied regardless of the harmonic analysis involved. It turns out that that the mode solutions of non scalar fields under certain circumstances experience infrared instability periods not known for scalar fields; the author is yet not sure about the physical essence of this phenomenon. Apart from this, traditionally infrared integrability issues arise when integrating modes over the spectrum $\operatorname{Spec}\left(D_{\Sigma}\right)$ of a Schrödinger operator $D_{\Sigma}$ if $\overline{\operatorname{Spec}\left(D_{\Sigma}\right)}$ includes the eigenvalue 0 [4. Here this question of integrability of modes over the spectrum is settled by showing that the suitably chosen mode solutions remain well under control even at non positive spectral values. As an example of application and as a byproduct the explicit formula of the propagator of the field in the Fourier space is found, which generalizes one obtained in [5].

Another advantage of geometric symmetries is the possibility to apply harmonic analysis. This is particularly true for the cosmological models where a rather rich 
group of spatial isometries is imposed. Then the spatial sections of the spacetime can be considered as homogeneous spaces, and the spatial Fourier transform can be investigated in much more detail with many explicit consequences. These properties then can be dragged to the time dependent Fourier transform and hence to the mode decomposition. Of particular interest in the quantum field theory on cosmological spacetimes are the spatially symmetric (invariant) states, of which the 2-point functions are bi-distributional solutions of the field equation which are invariant under the full isometry group. In $[5]$ the Fourier image of the isotropic homogeneous states of the Klein-Gordon field on FRW spacetime has been obtained, under an additional continuity requirement which has no clear physical interpretation. In contrast the most general form of invariant scalar bi-distributions on $\mathbb{R}^{d}$ has been obtained in [6] using a nice technique. What appears to be missing is a generalization of these results to sufficiently many homogeneous spaces so that practically all cosmological situations are covered. The harmonic analysis of FRW symmetry groups is well known since long, but strictly speaking isotropy is not as fundamental in cosmology as homogeneity, and one is also interested in cosmological models which are only homogeneous (Bianchi models) or in addition only partially isotropic (LRS models). The isometry groups of these spaces are described by Bianchi groups with their quotients and semidirect extensions (in case of LRS models). Some of these groups are solvable, others are semisimple, with finite or infinite center. Therefore it is not easy to establish a unified harmonic analytical approach for all cases, although one has to admit that Kirillov's theory for the solvable groups and Helgason's theory for semisimple groups together would cover the majority of situations. To obtain a unified theory one can adopt abstract harmonic analysis. This beautiful branch of mathematics allows to obtain many results in an admirable generality. However, apart from compact groups, it is not completely clear how to relate the abstract group Fourier transform with the eigenfunction expansion of the invariant Laplace operator. At least there seems to be no unified exposition of these techniques applied in the cosmological context.

The aim of our second part will be to put together some tools from harmonic analysis which are adequate in cosmology, and to obtain useful results with their help. First a short survey on homogeneous bundles in general, and on semidirect homogeneous bundles in particular is carried out, and it is shown that they cover the vast and the most important majority of the realistic cosmological structures. Next, the abstract harmonic analytical Fourier transform is introduced with its requisites on semidirect homogeneous spaces. Although the abstract Fourier transform on groups and the representation theory in homogeneous spaces are to be considered as well studied and widely known, the abstract Fourier transform on homogeneous spaces is not that popular and deserves a better exposition (at least we were not able to find a satisfactory one in the literature). Then some properties of the Fourier transformed distributions are established. Although some of these results may be known to experts in harmonic analysis, we were not able to locate them in the for required for our purposes in the literature. And because these prop- 
erties are needed to obtain our results on invariant distributions they are included here with proofs. Next an attempt is made to unify the abstract Fourier transform and the usual spatial Fourier transform given by the eigenfunction expansion of the Laplace operator. Several remarks are made on this way, which may serve as a guideline to completely build the desired correspondence once a particular structure is specified. This has been indeed performed for the purely homogeneous cosmological models, which will appear in a subsequent publication. The necessity of such an explicit correspondence consists in the ability to transfer the results obtained in the abstract setup to the situation with the usual Fourier transform, which is far more useful a tool for concrete calculations. Finally, by a generalization of the above mentioned technique in [6] the general form of the invariant bi-distributions in arbitrary semidirect homogeneous vector bundle is found without any additional assumptions on their regularity. It is concluded that the polynomial bound of the Fourier transformed homogeneous state as found in [5 is a consequence of the imposed regularity requirements.

\section{Mode Decomposition of Hyperbolic Fields}

\subsection{Linear hyperbolic fields}

It is generally believed that the forces of nature are described by tensor and spinor fields. A geometrical generalization of those are the vector bundle fields, i.e., fields as smooth sections of some vector bundles. In general relativity one works mainly on a four dimensional Lorentzian smooth manifold $(M, g)$ which is called a spacetime. We will be concerned with hyperbolic fields given by a wave equation, hence we put an additional constraint on the spacetime $(M, g)$ to be globally hyperbolic, so that the Cauchy problem of the wave equation is well-posed. For simplicity only linear fields will be discussed here. For the reduction of the Maxwell and Proca fields to linear hyperbolic fields the reader is referred to [4, [7]. We summarize the basic setup of the the linear hyperbolic fields in the following section.

Let $V$ be an $n$-dimensional vector space. Let $\mathcal{T} \stackrel{\pi}{\rightarrow} M$ be a vector bundle with standard fiber $V$ and with a pseudo-Riemannian metric $\langle u, v\rangle_{\mathfrak{g}}$. Let further $\mathcal{E}(\mathcal{T})=$ $C^{\infty}(\mathcal{T})$ and $\mathcal{D}(\mathcal{T})=C_{0}^{\infty}(\mathcal{T})$ be the spaces of smooth sections and of those with compact support, correspondingly. Let $\nabla$ be a metric connection on $\mathcal{T}$ and $\square^{\nabla}$ the associated d'Alambert operator on $\mathcal{E}(\mathcal{T})$. Define the field operator to be the normal hyperbolic operator $D=\square^{\nabla}+m^{\star}(x)$ acting on $\mathcal{E}(\mathcal{T})$, where $m^{\star} \in C^{\infty}(M)$ is a generalization of the usual mass term $m^{2}$ which now can also contain the coupling term $\xi R$. Note that because differential operators are support-decreasing, $D \mathcal{D}(\mathcal{T}) \subset \mathcal{D}(\mathcal{T})$. A free linear hyperbolic field $\phi \in \mathcal{E}(\mathcal{T})$ is a solution of the field equation $D \phi=0$.

Being a globally hyperbolic spacetime, $M=\mathcal{I} \times \Sigma$, where $\mathcal{I} \subseteq \mathbb{R}$ is an interval, and for each $t \in \mathcal{I}$ the hypersurface $\Sigma_{t} \sim \Sigma$ is a three dimensional embedded Riemannian submanifold, which is spacelike with respect to $g$ and is a Cauchy surface in the sense described below. Thanks to [8] one can choose a smooth global 
time function $t$ and a coordinate atlas such that $x=(t, \vec{x})=\left(x_{0}, x_{1}, x_{2}, x_{3}\right)$ where $t \in \mathcal{I}$ and $\vec{x} \in \Sigma$, i.e., $\Sigma_{t}$ are equal $t$ hypersurfaces. The restriction of the bundle $\mathcal{T}$ to the submanifold $\Sigma_{t}$ will be denoted by $\mathcal{T}_{t}=\pi^{-1}\left(\Sigma_{t}\right)$. The spaces of smooth sections will be $\mathcal{E}\left(\mathcal{T}_{t}\right)=C^{\infty}\left(\mathcal{T}_{t}\right)$ and $\mathcal{D}\left(\mathcal{T}_{t}\right)=C_{0}^{\infty}\left(\mathcal{T}_{t}\right)$. If $i_{t}: \mathcal{T}_{t} \rightarrow \mathcal{T}$ is the identical embedding, then its pullback $i_{t}^{*}$ is the restriction map for objects on $\mathcal{T}$ to $\mathcal{T}_{t}$. In particular $i_{t}^{*}: \mathcal{E}(\mathcal{T}) \rightarrow \mathcal{E}\left(\mathcal{T}_{t}\right)$ and $i_{t}^{*}: \mathcal{D}(\mathcal{T}) \rightarrow \mathcal{D}\left(\mathcal{T}_{t}\right)$ are linear surjective maps. The embedding $\pi \circ i_{t} \circ \pi^{-1}: M \rightarrow \Sigma$ gives rise to a natural embedding $i_{t}: T M \rightarrow T \Sigma$ and of all tensor bundles (using the same symbols $i_{t}, i_{t}^{*}$ for different restrictions in the spirit of polymorphism should not lead to a confusion). The Riemannian metric $h$ on $T \Sigma$ will be $h=-i_{t}^{*}(g)$, with minus sign here because of the signature convention $(+,-,-,-)$. The restriction $i_{t}^{*}(\nabla)=\nabla_{i_{t}^{*}(.)}=\nabla^{t}$ is a metric connection on $\mathcal{T}_{t}$. The associated Laplace operator $\Delta_{t}=\Delta \nabla^{t}$ is an elliptic operator on $\mathcal{E}\left(\mathcal{T}_{t}\right)$ (so that $-\Delta_{t}$ is a positive operator). The restriction of the field operator $D$ to $\mathcal{E}\left(\mathcal{T}_{t}\right)$ will be denoted by $D_{\Sigma_{t}}=-\Delta_{t}+m^{\star}(x)$.

An existence and uniqueness theorem [7, $[9,[10$ for wave operators tells that the Cauchy problem is well posed: there exists a bijective linear map

$$
\mathcal{E}\left(\mathcal{T}_{t}\right) \oplus \mathcal{E}\left(\mathcal{T}_{t}\right) \ni\left(f_{0}, f_{1}\right) \rightarrow \jmath\left(f_{0}, f_{1}\right) \in\{f \in \mathcal{E}(\mathcal{T}): D f=0\}
$$

such that $f_{0}=i_{t}^{*}(f)$ and $f_{1}=i_{t}^{*}\left(\nabla_{t} f\right)$, where $\nabla_{t}=\nabla_{\frac{\partial}{\partial t}}$. Furthermore, there exist unique Green's operators $E^{ \pm}: \mathcal{D}(\mathcal{T}) \rightarrow \mathcal{E}(\mathcal{T})$ satisfying $D E^{ \pm}=E^{ \pm} D=i d_{\mathcal{D}(\mathcal{T})}$ and $\operatorname{supp}\left\{G^{ \pm} f\right\} \subset J^{ \pm}(\operatorname{supp}\{f\})$ for all $f \in \mathcal{D}(\mathcal{T})$. Here $J^{ \pm}(N)$ with a subset $N \subset M$ denotes the causal future/past of $N$. Define by $E=E^{+}-E^{-}$the propagator of $D$, which satisfies $D E=E D=0$. Now $\operatorname{Sol}(\mathcal{T})=\jmath\left(\mathcal{E}\left(\mathcal{T}_{t}\right) \oplus \mathcal{E}(\mathcal{T})_{t}\right)$ and $\operatorname{Sol}_{0}(\mathcal{T})=$ $\jmath\left(\mathcal{D}\left(\mathcal{T}_{t}\right) \oplus \mathcal{D}\left(\mathcal{T}_{t}\right)\right)$ will denote correspondingly the spaces of all smooth solutions, and of those satisfying $\operatorname{supp}\{f\} \cap \Sigma_{t}$ compact for all $t \in \mathcal{I}$, respectively. Then $E \mathcal{D}(\mathcal{T}) \subset \operatorname{Sol}_{0}(\mathcal{T})$. There is a symplectic form on $\operatorname{Sol}_{0}(\mathcal{T})$ :

$$
\sigma(u, v)=\int_{\Sigma_{t}} d \mu_{h}\left[\left\langle i_{t}^{*}(u), i_{t}^{*}\left(\nabla_{t} v\right)\right\rangle_{g}-\left\langle i_{t}^{*}\left(\nabla_{t} u\right), i_{t}^{*}(v)\right\rangle_{g}\right], \forall u, v \in \operatorname{Sol}_{0}(\mathcal{T}), \forall t \in \mathcal{I},
$$

where $h=-i_{t}^{*}(g)$ is the induced Riemannian metric on $\Sigma_{t}$. That this is conserved (analogous to a Wronskian in ODE) can be seen by considering the Green's identity for $u, v \in \operatorname{Sol}_{0}(\mathcal{T})$ on the regular cylindric region $U=\left(t_{1} ; t_{2}\right) \times \Sigma \subset M$ for any $t_{1} \neq t_{2}$,

$$
\begin{gathered}
0=\int_{U} d \mu_{g}\left[\langle u, D v\rangle_{g}-\langle D u, v\rangle_{g}\right]= \\
=\int_{\partial U} d \mu_{h}\left[\left\langle i_{t}^{*}(u), i_{t}^{*}\left(\nabla_{t} v\right)\right\rangle_{g}-\left\langle i_{t}^{*}\left(\nabla_{t} u\right), i_{t}^{*}(v)\right\rangle_{g}\right], \forall u, v \in \operatorname{Sol}_{0}(\mathcal{T}) .
\end{gathered}
$$

This identity also helps us along with Green's operators to find the explicit form of the map J. Given any $v \in \operatorname{Sol}_{0}(\mathcal{T}), f \in \mathcal{D}(\mathcal{T})$, we apply it two times; once for the pair $v, u=E^{+}(f)$ on the region $U^{+}=(-\inf \{\mathcal{I}\} ; t)$ and once for the 
pair $v, u=E^{-}(f)$ on the region $U^{+}=(t ; \inf \{\mathcal{I}\})$. Summing up the resulting two identities and using the support properties of $E^{ \pm}$we arrive at

$$
\int_{M} d \mu_{g}\langle v, f\rangle_{g}=\sigma(v, E(f))
$$

We see that the functional $\sigma(v, E()):. \mathcal{D}(\mathcal{T}) \rightarrow \mathbb{C}$ actually is given by a smooth integral kernel, which equals $v$. Thus we can write symbolically

$\jmath\left(f_{0}, f_{1}\right)[y]=\int_{\Sigma_{t}} d \mu_{h}\left[\left\langle f_{0}, \nabla_{t} E(y)\right\rangle_{g}-\left\langle f_{1}, E(y)\right\rangle_{g}\right], \forall f_{0}, f_{1} \in \mathcal{D}\left(\mathcal{T}_{t}\right), y \in M, t \in \mathcal{I}$.

For full details of this last computation the reader is referred to [11, where the argument is given for 1-forms, but is readily applicable to our more general case.

Proposition 2.1. The operator $E: \mathcal{D}(\mathcal{T}) \rightarrow \operatorname{Sol}_{0}(\mathcal{T})$ is surjective.

Proof. Let $v \in S o l_{0}$, and let $K_{v}=\operatorname{supp} v \cap([0,1] \times \Sigma)$ be the compact region of its support between times 0 and 1. Let further $\chi \in \mathcal{E}(M)$ be a smooth function which equals 1 for $t<0$ and 0 for $t>1$. Denote $v^{-}=-v \chi$ and $v^{+}=v(1-\chi)$, then $v=v^{+}-v^{-}$. Let $f_{v}=D v^{+}$, then $\operatorname{supp} f_{v} \subset K_{v}$ is compact, hence $f_{v} \in \mathcal{D}(\mathcal{T})$. The equation $f_{v}=D v^{+}$has a unique solution with past compact support, and it is given by $v^{+}=E^{+} f_{v}$. Now $D v^{-}=-D v+D v^{+}=f_{v}$, and similarly $v^{-}=E^{-} f_{v}$. Then $v=E^{+} f_{v}-E^{-} f_{v}=E f_{v}$. The arbitrariness of $\chi$ reflects the non-injectivity of $E$.

\subsection{Spectral mode decomposition}

Henceforth we will use nomenclature introduced in the Appendix A without special notice. Consider the operators $D: \mathcal{D}(\mathcal{T}) \rightarrow \mathcal{D}(\mathcal{T})$ and $D_{\Sigma}: \mathcal{D}\left(\mathcal{T}_{t}\right) \rightarrow \mathcal{D}\left(\mathcal{T}_{t}\right)$. If $m^{\star}(x) \in \mathbb{R}$ everywhere on $M$, then by the virtue of Green's identity $D$ and $D_{\Sigma_{t}}$ are formally self-adjoint with respect to the inner products $(,)_{M}$ and $(,)_{\Sigma_{t}}$. We will not need the self-adjointness of $D$ in the current work. The constructions below will pertain mainly to $D_{\Sigma_{t}}$. The conditions on $m^{\star}(x)$ for $D_{\Sigma_{t}}$ to have a self-adjoint extension can be found in [12. We moreover require that the operator $D_{\Sigma_{t}}$ be lower semi-bounded. In practice will be mainly interested in cosmological models, where $m^{\star}(x)=m^{\star}(t)$ is a function of time only, so that no problems arise. Below we assume self-adjoint extensions for both $D$ and $D_{\Sigma_{t}}$, but for $D$ this is only symbolic and targets simply at coherent notations.

Let $D$ and $D_{\Sigma_{t}}$ be extended to self-adjoint operators on $L^{2}(\mathcal{T})$ and $L^{2}\left(\mathcal{T}_{t}\right)$ respectively. In the rigged Hilbert spaces [6], 13, 14] $\mathcal{D}(\mathcal{T}) \subset L^{2}(\mathcal{T}) \subset \mathcal{D}(\mathcal{T})^{\prime}$ and $\mathcal{D}\left(\mathcal{T}_{t}\right) \subset L^{2}\left(\mathcal{T}_{t}\right) \subset \mathcal{D}\left(\mathcal{T}_{t}\right)^{\prime}$ operators $D$ and $D_{\Sigma_{t}}$ possess complete systems of eigenfunctions $\left\{u_{\rho}\right\}$ and $\left\{\zeta_{\lambda}\right\}$ satisfying

$$
\begin{gathered}
D u_{\rho}=\rho u_{\rho}, u_{\rho} \in \mathcal{D}(\mathcal{T})^{\prime}, \rho \in \mathbb{R} \\
D_{\Sigma_{t}} \zeta_{\lambda}=\lambda \zeta_{\lambda}, \zeta_{\lambda} \in \mathcal{D}\left(\mathcal{T}_{t}\right)^{\prime}, \lambda \in \mathbb{R} .
\end{gathered}
$$


Denote by $\mathcal{D}(\mathcal{T})_{\rho}^{\prime}$ and $\mathcal{D}\left(\mathcal{T}_{t}\right)_{\lambda}^{\prime}$ the linear spaces of eigenfunctions corresponding to $\rho$ and $\lambda$, respectively. Furthermore, there exists an isomorphism

$$
L^{2}\left(\mathcal{T}_{t}\right)=\int_{\mathbb{R}}^{\oplus} d \nu(\lambda) H(\lambda)
$$

where

$$
\left.D_{\Sigma_{t}}\right|_{H(\lambda)}=\lambda
$$

and $d \nu(\lambda)$ is a positive measure. Each $H(\lambda)$ is continuously embedded in $\mathcal{D}\left(\mathcal{T}_{t}\right)_{\lambda}^{\prime}$.

The eigenfunction expansion of $D$ will be the map

$$
\mathcal{D}(\mathcal{T}) \ni f \rightarrow \tilde{f}_{\rho} \in\left(\mathcal{D}(\mathcal{T})_{\rho}^{\prime}\right)^{\prime *}, \rho \in \mathbb{R},
$$

$\left(X^{\prime *}\right.$ denotes the space of continuous antilinear functionals on the space $X$ ) where $\tilde{f}_{\rho}$ is defined by

$$
\tilde{f}_{\rho}\left(u_{\rho}\right)=\bar{u}_{\rho}(f), \forall f \in \mathcal{D}(\mathcal{T}), u_{\rho} \in \mathcal{D}(\mathcal{T})_{\rho}^{\prime} .
$$

(Here we defer a little from Gelfand's notations who puts $\tilde{f}_{\rho}\left(u_{\rho}\right)=u_{\rho}(f)$.) The expansion of $D_{\Sigma_{t}}$ on $\mathcal{D}\left(\mathcal{T}_{t}\right)$ is constructed similarly. Note that $D_{\Sigma_{t}}$ is an elliptic operator, hence $\mathcal{D}\left(\mathcal{T}_{t}\right)_{\lambda}^{\prime} \subset \mathcal{E}\left(\mathcal{T}_{t}\right)$.

If each $\mathcal{D}\left(\mathcal{T}_{t}\right)_{\lambda}^{\prime}$ is finite dimensional (eigenvalue $\lambda$ has a finite multiplicity $N_{\lambda}$ ), then

$$
H(\lambda)=\mathcal{D}\left(\mathcal{T}_{t}\right)_{\lambda}^{\prime}, \operatorname{dim} H(\lambda)=N_{\lambda}
$$

Choose $\left\{\zeta_{\lambda, i}\right\}_{i=1}^{N_{\lambda}}$ be a an orthonormal basis in $\mathcal{D}\left(\mathcal{T}_{t}\right)_{\lambda}^{\prime}$ (orthonormality understood in $H(\lambda))$. Then $\left(\mathcal{D}\left(\mathcal{T}_{t}\right)_{\lambda}^{\prime}\right)^{\prime *} \sim \mathbb{C}^{N_{\lambda}}$ by the bijective linear map

$$
\tilde{f}\left(\zeta_{\lambda}\right)=\tilde{f}\left(\sum_{i=1}^{N_{\lambda}} c_{i} \zeta_{\lambda, i}\right) \rightarrow\left\{\tilde{f}_{i}=\tilde{f}\left(\zeta_{\lambda, i}\right)\right\}_{i=1}^{N_{\lambda}}, \forall \tilde{f} \in\left(\mathcal{D}\left(\mathcal{T}_{t}\right)_{\lambda}^{\prime}\right)^{\prime *},
$$

where each $\tilde{f}_{i} \in \mathbb{C}$. In particular, if $\tilde{f}_{\lambda}$ is the mode expansion of $f \in \mathcal{D}\left(\mathcal{T}_{t}\right)$, then the map

$$
\mathcal{D}\left(\mathcal{T}_{t}\right) \ni f \rightarrow \tilde{f}_{\lambda} \rightarrow\left\{\tilde{f}_{\lambda, i}\right\} \in \int_{\mathbb{R}}^{\oplus} d \nu(\lambda) \mathbb{C}^{N_{\lambda}}
$$

will serve as a Fourier transform on $\mathcal{D}\left(\mathcal{T}_{t}\right)$. Define

$$
\operatorname{Spec}\left\{D_{\Sigma_{t}}\right\}=\operatorname{supp}\{d \nu\}
$$

and

$$
\tilde{\Sigma}=\left\{(\lambda, i): \lambda \in \operatorname{Spec}\left\{D_{\Sigma_{t}}\right\}, i=1, \ldots, N_{\lambda}\right\} .
$$

Define the spectral measure on $\tilde{\Sigma}$ as

$$
d \mu(\alpha)=d \nu(\lambda) \times d \sharp(i),
$$

where $d \sharp$ is the counting measure. The map (Eq 2.3 ) can be reformulated as

$$
\tilde{f}(\alpha)=\mathcal{F}[f](\alpha), f \in \mathcal{D}\left(\mathcal{T}_{t}\right) .
$$


Then the formula (Eq2.2) arises a Plancherel formula

$$
(f, h)_{\Sigma_{t}}=\int_{\tilde{\Sigma}} d \mu(\alpha) \overline{\tilde{f}}(\alpha) \tilde{g}(\alpha),
$$

and a Peter-Weyl (or Fourier inversion) formula

$$
f(x)=\int_{\tilde{\Sigma}} d \mu(\alpha) \tilde{f}(\alpha) \zeta_{\alpha}(x),
$$

which holds in the $L^{2}$-sense so far. In our cases of interest this convergence will be in the compact topology.

However, if $\mathcal{D}\left(\mathcal{T}_{t}\right)_{\lambda}^{\prime}$ is infinite dimensional, more delicate tools are needed to obtain a Fourier transform with desired properties. Such tools naturally include an investigation of symmetries of the underlying geometrical structure, and this is the subject of the harmonic analysis. We will often use the formal structure (Eq2.4) without mentioning a concrete realization, assuming that this is possible. For the cases of our interest we will indeed find a realization by means of adapted Fourier transform.

In the theory of Fourier transform, and in particular in the Euclidean case, the Paley-Wiener theorems describe the functional analytical structure of the image $\mathcal{F}\left[\mathcal{D}\left(\mathcal{T}_{t}\right)\right]$ of the test function space under the action of the Fourier transform. This description is very useful when analyzing the situation in the Fourier space. Unfortunately there is no (at least known to us) general Paley-Wiener argument valid for any Fourier transform arisen in this manner, and the proofs of the existing ones are rather structure-specific. In applications we would like, however, to obtain results which are valid in a large variety of cases, and therefore we will introduce a notion of 'conventional' Fourier transform which consists of a number of assumptions pertaining to the analytical properties of a given Fourier transform. Some of our later results will be valid under the assumption that the eigenvalue expansion of the operator $\Delta_{t}$ has at least some of the properties of a conventional Fourier transform. One says that a good definition is an assumption of a theorem. In this sense the following is not a good definition as we will not manage to use all properties in this work. However it seems feasible that these properties will become useful for several applications in quantum field theory.

Definition 2.1. A Fourier transform $\mathcal{F}$ given by the eigenfunction expansion against a complete system $\left\{\zeta_{\alpha}\right\}_{\alpha \in \tilde{\Sigma}}$ will be called conventional if

(i) The Fourier space (or momentum space) $\tilde{\Sigma}$ is a manifold consisting of $n=\operatorname{dim} V$ components, $\tilde{\Sigma}=\bigcup_{i=1}^{n} \tilde{\Sigma}^{i}$, and each component $\tilde{\Sigma}^{i}$ is either a discreet set or an (not necessarily connected) analytical manifold

(ii) The eigenvalue $\lambda(\alpha)$ is an analytic function on $\tilde{\Sigma}$

(iii) The range $\mathcal{F}\left[\mathcal{D}\left(\mathcal{T}_{t}\right)\right]$ is a subspace of the space of analytic functions $\tilde{f}(\alpha)$ on $\tilde{\Sigma}$ with rapid decay in $\lambda$

(iv) There is an involution $\alpha \rightarrow-\alpha$ on $\tilde{\Sigma}$ such that $\zeta_{-\alpha}=\bar{\zeta}_{\alpha}$. 
Note that it follows $\lambda(-\alpha)=\lambda(\alpha)$. In later sections we will give harmonic analytical justifications for such a 'conjecture'. This conjecture is anticipated, in particular, for all cosmological models. Moreover, being true for FRW spaces, it can be proven also for Bianchi I-VII spaces (to appear in a future publication).

Further we will be mainly interested in the space of weak solutions of the field equation, $\mathcal{D}(\mathcal{T})_{0}^{\prime}$, and will try to find a convenient characterization of it. In particular we will be looking for a complete system of solutions $\left\{u_{\alpha}\right\}$ spanning $\mathcal{D}(\mathcal{T})_{0}^{\prime}$ and being in addition well handled (i.e., smooth, explicit etc.). One means of doing this is to look at a subspace of $\mathcal{D}(\mathcal{T})_{0}^{\prime}$ which consist of solutions $f(x)=a(t) b(\vec{x})$, $a \in C^{\infty}(\mathcal{I}), b \in \mathcal{E}\left(\mathcal{T}_{t}\right)$. Then under fortunate circumstances the field equation breaks apart into two lower dimensional elliptical eigenproblems, which are much easier to deal with. Which are those circumstances and whether such solutions span $\mathcal{D}(\mathcal{T})_{0}^{\prime}$, and related questions, are the matter of the problem of variable separation. In the next sections we will find out in which cases this is possible and how to perform it.

\subsection{Separation of variables}

As discussed above, we would like to span the space $\mathcal{D}(\mathcal{T})_{0}^{\prime}$ of weak solutions of the field equation by a family of easily computable smooth solutions $\left\{u_{\alpha}\right\}$. In this section we will see when and how one can perform this for the smooth solutions $\operatorname{Sol}_{0}(\mathcal{T})$. The necessary requisites for this will be predominantly geometric requirements. In the next section we will show that under additional functional analytical assumptions the procedure can be extended to $\mathcal{D}(\mathcal{T})_{0}^{\prime}$ in a natural way.

Definition 2.2. Let $S$ be a subspace of $\mathcal{E}(\mathcal{T})$ with closure $\bar{S} \supseteq S, \mathfrak{M}$ a measure space with measure $d \mathfrak{m}$. An $\mathfrak{M}$-measurable family $\left\{u_{\alpha}\right\}_{\alpha \in \mathfrak{M}}$ of elements $u_{\alpha} \in \bar{S}$ will be called a complete or spanning system for $S$ if for any $v \in S$ there exists a unique (modulo null-supported functions) $\mathfrak{M}$-measurable function $a^{v}: \mathfrak{M} \rightarrow \mathbb{R}$ $\left(a^{v}: \mathfrak{M} \rightarrow \mathbb{C}\right)$ such that

$$
v=\int_{\mathfrak{M}} d \mathfrak{m}(\alpha) a^{v}(\alpha) u_{\alpha} .
$$

For the details on integration of nuclear Frechét space-valued functions see [15] and references therein. We will always take $\mathfrak{M}$ to be minimal, i.e., there exists no subset $A \subset \mathfrak{M}$ with $\mathfrak{m}(A)>0$ such that $a^{v}(A)=0$ for all $v \in S$. If the uniqueness

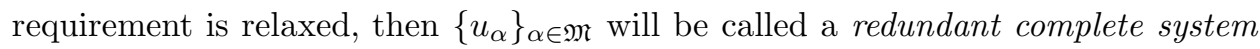
for $S$. Note that from the uniqueness property it follows, that for $d \mathfrak{m}$-almost all $\alpha \in \mathfrak{M}$, there exists no $\alpha \neq \beta \in \mathfrak{M}$ with $u_{\alpha}+p(\alpha) u_{\beta}=0, p(\alpha) \neq 0$ a number. In other words, almost all $u_{\alpha}$ are pairwise independent.

$\mathcal{E}(\mathcal{T})$ is a closed topological vector space with the topology of compact convergence, and $\operatorname{Sol}(\mathcal{T})$ and $\operatorname{Sol}_{0}(\mathcal{T})$ are linear subspaces. A spanning system $\left\{u_{\alpha}\right\}_{\alpha \in \mathfrak{M}}$ of $\operatorname{Sol}_{0}(\mathcal{T})$ of the form $u_{\alpha}=T_{\alpha} X_{\alpha}$, where $T_{\alpha} \neq \bar{T}_{\alpha} \in C^{\infty}(\mathcal{I})\left(T_{\alpha}\right.$ and $\bar{T}_{\alpha}$ linearly independent) and $X_{\alpha} \in \mathcal{E}\left(\mathcal{T}_{t}\right)$, such that $D T_{\alpha} X_{\alpha}=D \bar{T}_{\alpha} X_{\alpha}=0$, will be called a 
complete (time-)variable separated system of solutions (or shorter, separating system).

We will assume that a Fourier transform $\mathcal{F}$ on $\mathcal{D}\left(\mathcal{T}_{t}\right)$ is specified by means of the spectral decomposition of $D_{\Sigma_{t}}$ as described in the previous section. The system of eigenfunctions $\left\{\zeta_{\alpha}^{t}\right\}$ of $\Delta_{t}$, with the Fourier space $\tilde{\Sigma}_{t}$ and the spectral measure $d \mu(\alpha)$ on it, provide a spanning system for $\mathcal{D}\left(\mathcal{T}_{t}\right)$ by means of the Fourier inversion (or Peter-Weyl) formula. Below we will come across the question of a spectral theory of formally non-self-adjoint, i.e., asymmetric differential operators of type $a(x) D_{\Sigma_{t}}$. As a rule, the eigenfunction problems of asymmetric (aside from unitary) operators are ill-posed, and eigenfunctions do not comprise a complete system, but there are rare exceptions. At this point we have to admit the non-exhaustiveness of our treatment, as we do not analyze this possibility. We will loosely rule out the possibility of such operators to have a well-posed eigenfunction problem.

A small remark will be useful later in the section.

Remark 2.1. If $\left\{T_{\alpha} X_{\alpha}\right\}_{\alpha \in \mathfrak{M}}$ is a separating system for $\operatorname{Sol}_{0}(\mathcal{T})$ with compact

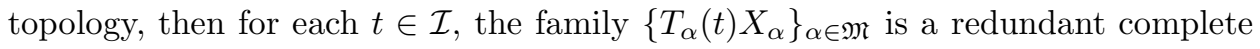
system for $\mathcal{D}\left(\mathcal{T}_{t}\right)$. In particular, for each $\vec{x} \in \Sigma_{t}$, the family $\left\{X_{\alpha}(\vec{x})\right\}_{\alpha \in \mathfrak{M} \text { contains }}$ a (possibly redundant) basis of $V$.

The assertions are relatively obvious in the view of the fact, that the restriction maps $i_{t}^{*}, i_{t}^{*} \circ \nabla_{t}: \operatorname{Sol}_{0}(\mathcal{T}) \rightarrow \mathcal{D}\left(\mathcal{T}_{t}\right)$ are surjective, and hence a spanning system for $\operatorname{Sol}_{0}(\mathcal{T})$ must give a redundant complete system for the Cauchy data $\mathcal{D}\left(\mathcal{T}_{t}\right) \oplus \mathcal{D}\left(\mathcal{T}_{t}\right)$ on $\Sigma_{t}$.

Remark 2.2. Let two equations $\ddot{T}(t)+F(t) \dot{T}(t)+G(t) T(t)=0$ and $\ddot{T}(t)+$ $H(t) \dot{T}(t)+J(t) T(t)=0$ have two common linearly independent solutions $T(t)$ and $S(t)$. Then by Liouville formula the Wronski determinant $\operatorname{det} W[T, S](t)$ evolves by

$$
\operatorname{det} W[T, S](t)=\operatorname{det} W[T, S](0) e^{-\int_{0}^{t} d \tau F(\tau)}=\operatorname{det} W[T, S](0) e^{-\int_{0}^{t} d \tau H(\tau)},
$$

hence $F=H$ and thereby also $G=J$.

Proposition 2.2. The solution space $\operatorname{Sol}_{0}(\mathcal{T})$ admits a separating system if and only if there exists a covering of $\mathcal{T}$ by local trivializations such that the following local conditions are satisfied everywhere (metric $g$ is time-separated):

(i) $g_{00}=g_{00}(t)$, the metric component $g_{00}$ depends only on time

(ii) the expression $\sum_{i, j=1}^{3} g^{i j}(x) \frac{\partial g_{i j}}{\partial t}(x)$ is a function of time only

(iii) the connection 1-form $\Gamma$ and Christoffel symbols $\Gamma_{i j}^{k}$ satisfy

$$
\begin{gathered}
\sum_{i=1}^{3} g^{i j}\left[\Gamma_{0}, \Gamma_{i}\right]=0, \forall j>0 \\
\sum_{i, j=1}^{3} g^{i j}\left[\Gamma_{0}, \frac{\partial \Gamma_{j}}{\partial x^{i}}+\Gamma_{i} \Gamma_{j}-\sum_{k=0}^{3} \Gamma_{i j}^{k} \Gamma_{k}\right]=0
\end{gathered}
$$




$$
\Gamma_{0}=\Gamma_{0}(t) \text { is a function of time only }
$$

(iv) the eigenfunction problem of $D_{\Sigma_{t}}$ on different $\Sigma_{t}$ can be adjusted, so that all $\tilde{\Sigma}_{t}$ are isomorphic and the eigenfunctions $\zeta_{\alpha}^{t}=\zeta_{\alpha}$ are time-independent.

Proof. Throughout the section we will work exclusively locally, i.e., in a local trivialization $\pi^{-1}(U) \stackrel{\Psi}{\rightarrow} U \times V, U \subset M$. Thus we identify the sections in a bundle having a typical fiber $\mathfrak{F}$ with functions in $C^{\infty}(U ; \mathfrak{F})$. We will not keep the flag $U$ in this section but will always understand objects as restricted to $U$.

The d'Alambert operator $\square^{\nabla}$ on $\mathcal{E}(\mathcal{T})$ has the following local expression in terms of the connection form coefficients $\Gamma_{i}$ and Christoffel symbols $\boldsymbol{\Gamma}_{i j}^{k}$,

$$
\square^{\nabla}=\sum_{i, j=0}^{3} g^{i j}\left[\frac{\partial^{2}}{\partial x^{i} \partial x^{j}}+2 \Gamma_{i} \frac{\partial}{\partial x^{j}}-\sum_{k=0}^{3} \boldsymbol{\Gamma}_{i j}^{k} \frac{\partial}{\partial x^{k}}+\frac{\partial \Gamma_{i}}{\partial x^{j}}+\Gamma_{i} \Gamma_{j}-\sum_{k=0}^{3} \boldsymbol{\Gamma}_{i j}^{k} \Gamma_{k}\right]
$$

and the field operator $D$ locally looks like

$$
D=\sum_{i, j=0}^{3} g^{i j} \frac{\partial^{2}}{\partial x^{i} \partial x^{j}}+\sum_{i=0}^{3} A^{i} \frac{\partial}{\partial x^{i}}+B+m^{\star},
$$

where $A^{i}, B \in C^{\infty}(U, \operatorname{End}(V))$. To achieve a time separation we need to choose a coordinate atlas such that everywhere $g^{0 i}=0$ for $i>0$. Then the operator $D$ locally breaks apart into two differential operators, $D=D_{t}+D_{\Sigma_{t}}$, where

$$
D_{t}=g^{00} \frac{\partial^{2}}{\partial t^{2}}+A^{0} \frac{\partial}{\partial t}+B^{0}
$$

and

$$
D_{\Sigma_{t}}=\sum_{i, j=1}^{3} g^{i j} \frac{\partial}{\partial x^{i}} \frac{\partial}{\partial x^{j}}+\sum_{i=1}^{3} A^{i} \frac{\partial}{\partial x^{i}}+B^{3}+m^{\star}=-\Delta_{t}+m^{\star}
$$

is the restricted field operator defined earlier. $B^{0}, B^{3} \in C^{\infty}(U, \operatorname{End}(V))$ are to be seen explicitly from $(\mathrm{Eq} 2.5)$.

$\Rightarrow$ Necessity: Let $\left\{T_{\alpha} X_{\alpha}\right\}$ be the separating system system. Then

$$
\begin{array}{r}
D T_{\alpha}(t) X_{\alpha}(\vec{x})=\left(D_{t}+D_{\Sigma_{t}}\right) T_{\alpha}(t) X_{\alpha}(\vec{x})=\ddot{T}_{\alpha}(t) g^{00}(x) X_{\alpha}(\vec{x})+ \\
+\dot{T}_{\alpha}(t) A^{0}(x) X_{\alpha}(\vec{x})+T_{\alpha}(t)\left[B^{0}(x)+D_{\Sigma_{t}}\right] X_{\alpha}(\vec{x})=0 .
\end{array}
$$

That the metric signature is definite it follows that $g^{00}(x)$ never vanishes. We find a family of second order linear homogeneous differential equations

$$
\ddot{T}_{\alpha}(t) g^{00}(x) X_{\alpha}^{i}(\vec{x})+\dot{T}_{\alpha}(t)\left(A^{0}(x) X_{\alpha}(\vec{x})\right)^{i}+T_{\alpha}(t)\left(\left[B^{0}(x)+D_{\Sigma_{t}}\right] X_{\alpha}(\vec{x})\right)^{i}=0
$$

parameterized by the spatial coordinates $\vec{x} \in \Sigma$ and fiber indices $i=1, \ldots, n$. By definition we similarly have $D \bar{T}_{\alpha}(t) X_{\alpha}(\vec{x})=0$. This means that all these equations share at least two linearly independent solutions $T_{\alpha}$ and $\bar{T}_{\alpha}$. If for some $\vec{x}$ and $i$, 
$X_{\alpha}^{i}(\vec{x})=0$, then the existence of two linearly independent solutions for the resulting first order equation means that

$$
\left(A^{0}(x) X_{\alpha}(\vec{x})\right)^{i}=\left(\left[B^{0}(x)+D_{\Sigma_{t}}\right] X_{\alpha}(\vec{x})\right)^{i}=0 .
$$

Otherwise, by Remark 2.2 we find that there exist functions $F_{\alpha}, G_{\alpha} \in C^{\infty}(\mathcal{I})$ such that

$\left(A^{0}(x) X_{\alpha}(\vec{x})\right)^{i}=g^{00}(x) F_{\alpha}(t) X_{\alpha}^{i}(\vec{x}),\left(\left[B^{0}(x)+D_{\Sigma_{t}}\right] X_{\alpha}(\vec{x})\right)^{i}=g^{00}(x) G_{\alpha}(t) X_{\alpha}^{i}(\vec{x})$.

In both cases we establish that

$$
g_{00}(x) A^{0}(x) X_{\alpha}(\vec{x})=F_{\alpha}(t) X_{\alpha}(\vec{x})
$$

and

$$
g_{00}(x)\left[B^{0}(x)+D_{\Sigma_{t}}\right] X_{\alpha}(\vec{x})=G_{\alpha}(t) X_{\alpha}(\vec{x}) .
$$

Thus for each $t \in \mathcal{I}, X_{\alpha}$-s must be nothing else but the joint eigenfunctions of the operators $g_{00}(x) A^{0}(x)$ and $g_{00}(x)\left[B^{0}(x)+D_{\Sigma_{t}}\right]$ corresponding to eigenvalues $F_{\alpha}(t)$ and $G_{\alpha}(t)$, respectively. The operator $g_{00}(x) A^{0}(x)$ is simply a matrix, and at each point $x \in M$ has at most $n$ independent eigenvectors. By Remark 2.1, $X_{\alpha}(\vec{x})$ s span $V$, and thereby $\left\{X_{\alpha}\right\}_{\alpha \in \mathfrak{M}}$ contains bases of all eigenspaces of $g_{00}(x) A^{0}(x)$. From (Eq2.5) we find

$$
g_{00} A^{0}=2 \Gamma_{0}-g_{00} \sum_{i, j=0}^{3} g^{i j} \Gamma_{i j}^{0}
$$

and

$$
g_{00} B^{0}=\frac{\partial}{\partial t} \Gamma_{0}+\Gamma_{0}^{2}-\sum_{k=1}^{3} \boldsymbol{\Gamma}_{00}^{k} \Gamma_{k}-g_{00} \sum_{i, j=0}^{3} g^{i j} \boldsymbol{\Gamma}_{i j}^{0} \Gamma_{0} .
$$

Now turn to the eigenfunction problem (Eq2.8). As discussed above, for this problem to be well-posed it is necessary that the differential operator $g_{00}(x)\left[B^{0}(x)+D_{\Sigma_{t}}\right]$ is at least formally self-adjoint. But this is possible only if $g_{00}(x)=g_{00}(t)$, thus we have obtained the condition (i). Let us switch to an atlas, where the time function $t$ is redefined such that $g_{00}(t)=1$ (this step is not crucial, but only for convenience). It follows, that

$$
\Gamma_{00}^{k}=0, \forall k>0
$$

so we obtain

$$
\begin{array}{r}
A^{0}=2 \Gamma_{0}-\sum_{i, j=1}^{3} g^{i j} \Gamma_{i j}^{0}, \\
B^{0}=\frac{\partial}{\partial t} \Gamma_{0}+\Gamma_{0}^{2}-\sum_{i, j=1}^{3} g^{i j} \Gamma_{i j}^{0} \Gamma_{0} .
\end{array}
$$


Combining (Eq2.7) and (Eq2.10) we see that $\left\{X_{\alpha}\right\}$-s are the eigenvectors of $\Gamma_{0}$, and these eigenvectors are independent of $t$. Hence they are also the eigenvectors of $\frac{\partial}{\partial t} \Gamma_{0}$, and thus by (Eq 2.11) $A^{0}$ and $B^{0}$ are simultaneously triangularizable,

$$
B^{0} X_{\alpha}(\vec{x})=H_{\alpha}(x) X_{\alpha}(\vec{x}),
$$

for some $H_{\alpha} \in C^{\infty}(M)$. We note that

$$
\Gamma_{i j}^{0}=-\frac{1}{2} \frac{\partial g_{i j}}{\partial t}
$$

and denote

$$
P(x)=-\sum_{i, j=1}^{3} g^{i j}(x) \boldsymbol{\Gamma}_{i j}^{0}(x)=\frac{1}{2} \sum_{i, j=1}^{3} g^{i j}(x) \frac{\partial g_{i j}}{\partial t}(x) .
$$

Now (Eq 2.77) and (Eq2.8) tell us, that for each $t \in \mathcal{I}$ the operators $A^{0}$ and $D_{\Sigma_{t}}+$ $B^{0}$ have a common system of eigenfunctions spanning $\mathcal{D}\left(\mathcal{T}_{t}\right)$, and therefore must commute,

$$
\left[A^{0}, D_{\Sigma_{t}}+B^{0}\right] u=\left[A^{0}, D_{\Sigma_{t}}\right] u=0, \forall u \in \mathcal{D}\left(\mathcal{T}_{t}\right)
$$

This requires

$$
A^{0}(x)=2 \Gamma_{0}(x)+P(x)=A^{0}(t),
$$

and

$$
\begin{gathered}
\sum_{i=1}^{3} g^{i j}\left[\Gamma_{0}, \Gamma_{i}\right]=0, \forall j>0 \\
\sum_{i, j=1}^{3} g^{i j}\left[\Gamma_{0}, \frac{\partial \Gamma_{j}}{\partial x^{i}}+\Gamma_{i} \Gamma_{j}-\sum_{k=0}^{3} \Gamma_{i j}^{k} \Gamma_{k}\right]=0,
\end{gathered}
$$

exactly as the statement. Similarly, that operators $B^{0}$ and $B^{0}+D_{\Sigma_{t}}$ have the same eigenfunctions implies, that $\left[B^{0}, D_{\Sigma_{t}}\right]=0$, which on its turn requires $B^{0}(x)=$ $B^{0}(t)$, and thereby $P(x)=P(t)$ and $\Gamma_{0}(x)=\Gamma_{0}(t)$. Thus we have proven parts (ii) and (iii) of the statement. It follows further, that $H_{\alpha}(x)=H_{\alpha}(t)$, and thus the eigenfunction problem $(\mathrm{Eq} 2.8)$ becomes

$$
D_{\Sigma_{t}} X_{\alpha}(\vec{x})=\left(G_{\alpha}(t)-H_{\alpha}(t)\right) X_{\alpha}(\vec{x}) .
$$

This is exactly the eigenfunction problem of $D_{\Sigma_{t}}$, whence we conclude, that necessarily

$$
\left\{X_{\alpha}\right\}_{\alpha \in \mathfrak{M}} \subset\left\{\zeta_{\lambda}^{t}\right\}_{\lambda \in \mathbb{R}}
$$

Therefore

$$
G_{\alpha}(t)=H_{\alpha}(t)+\lambda_{\alpha}(t),
$$


where

$$
\lambda_{\alpha}(t)=\left\{\lambda \in \mathbb{R}: X_{\alpha} \in \mathcal{D}\left(\mathcal{T}_{t}\right)_{\lambda}^{\prime}\right\} .
$$

Now (Eq2.6) becomes

$$
\ddot{T}_{\alpha}(t)+F_{\alpha}(t) \dot{T}_{\alpha}(t)+G_{\alpha}(t) T_{\alpha}(t)=0,
$$

which is the mode equation for the mode $T_{\alpha}$. We have two spanning systems for

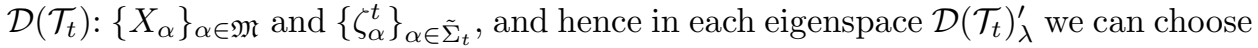

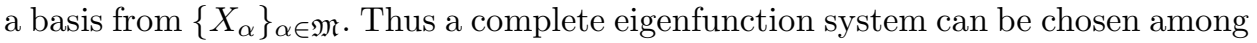
$\left\{X_{\alpha}\right\}_{\alpha \in \mathfrak{M}}$, proving the (iv) statement of the proposition. We are complete with the necessity.

$\Leftarrow$ Sufficiency: Suppose all the points of the statement are satisfied. Then, as we have seen above, by (iii) $A^{0}$ and $B^{0}$ are functions of $t$ having the same eigenvectors, and moreover, commute with $\Delta_{t}$. It follows that the actions of $A^{0}$ and $B^{0}$ preserve $\mathcal{D}\left(\mathcal{T}_{t}\right)_{\lambda}^{\prime}$, and thus by a Gramm-Schmidt operation the representatives $\zeta_{\alpha}$ can be chosen such that they are eigenfunctions of $A^{0}$ and $B^{0}$. Thus each $\tilde{\Sigma}_{\lambda}$, and thereby the entire $\tilde{\Sigma}$, decomposes into $n$ components corresponding to the eigendirections of $A^{0}$,

$$
\tilde{\Sigma}=\bigcup_{i=1}^{n} \tilde{\Sigma}^{i}
$$

For spatially homogeneous spacetimes discussed in later sections we will give a more conceptual justification of such a subdivision in terms of the representation theory.

Let for each $\alpha \in \tilde{\Sigma}$ choose a mode solution $T_{\alpha}$ of (Eq 2.12) arbitrarily (strictly speaking, not completely arbitrarily, but such that $T_{\alpha}$ and $\bar{T}_{\alpha}$ are linearly independent) and consider the union of two systems

$$
\{u, v\}_{\alpha \in \tilde{\Sigma}} \doteq\left\{u_{\alpha}\right\}_{\alpha \in \tilde{\Sigma}} \cup\left\{v_{\alpha}\right\}_{\alpha \in \tilde{\Sigma}}, u_{\alpha}=T_{\alpha} \zeta_{\alpha}, v_{\alpha}=\bar{T}_{\alpha} \zeta_{\alpha} .
$$

Choose any $\phi \in \operatorname{Sol}_{0}(\mathcal{T})$. Then for each $t \in \mathcal{I}$ the restriction $i_{t}^{*}(\phi)[\vec{x}]=\phi(t, \vec{x}) \in$ $\mathcal{D}\left(\mathcal{T}_{t}\right)$ can be Fourier expanded as

$$
\phi(t, \vec{x})=\int_{\tilde{\Sigma}} d \mu(\alpha) \hat{\phi}(\alpha ; t) \zeta_{\alpha}(\vec{x})
$$

with the integral converging in $L^{2}(\tilde{\Sigma}, \mu)$. Hence we can differentiate under the integral,

$$
\begin{aligned}
D \phi(t, \vec{x}) & =\int_{\tilde{\Sigma}} d \mu(\alpha) D\left[\hat{\phi}(\alpha ; t) \zeta_{\alpha}(\vec{x})\right]=\int_{\tilde{\Sigma}} d \mu(\alpha)[\ddot{\hat{\phi}}(\alpha ; t)+ \\
& \left.+F_{\alpha}(t) \dot{\hat{\phi}}(\alpha ; t)+G_{\alpha}(t) \hat{\phi}(\alpha ; t)\right] \zeta_{\alpha}(\vec{x})=0,
\end{aligned}
$$

where for convenience we again reparameterized $t$ to get $g_{00}=1$. Thus $\hat{\phi}(\alpha ; t)$ is a solution of the mode equation. All solutions of the ordinary second order equation (Eq 2.12) are smooth and comprise a two complex dimensional space,

$$
\hat{\phi}(\alpha ; t)=a_{\alpha}^{\phi} T_{\alpha}(t)+b_{\alpha}^{\phi} \bar{T}_{\alpha}(t), a_{\alpha}^{\phi}, b_{\alpha}^{\phi} \in \mathbb{C} .
$$


Inserting this into $(\mathrm{Eq} \sqrt{2.13})$ we finally arrive at

$$
\phi(t, \vec{x})=\int_{\tilde{\Sigma}} d \mu(\alpha)\left[a_{\alpha}^{\phi} T_{\alpha}(t) \zeta_{\alpha}(\vec{x})+b_{\alpha}^{\phi} \bar{T}_{\alpha}(t) \zeta_{\alpha}(\vec{x})\right],
$$

which exactly means, that $\{u, v\}_{\alpha \in \tilde{\Sigma}}$ is a separating system for $\operatorname{Sol}_{0}(\mathcal{T})$. (For compatibility with the definition one can concatenate $u_{\alpha}$ and $v_{\alpha}$ to a single function on the disjoint union $\tilde{\Sigma} \sqcup \tilde{\Sigma}$.)

The assertion of this proposition can be interpreted as follows. If a mode decomposition in a reasonable fashion exists for $\operatorname{Sol}_{0}(\mathcal{T})$ then it is basically the mode decomposition given by the time dependent Fourier transform which we will define a few paragraphs later.

As a supplement to the proposition we make a few remarks. Let $\hat{g}_{i j}=g\left(\partial_{i}, \partial_{j}\right)$ and $\hat{h}_{i j}=h\left(\partial_{i}, \partial_{j}\right)$ be the matrices of the metrics $g$ and $h$, correspondingly, in a local chart, and $\sigma_{k}(\hat{h})$ the eigenvalues of the symmetric matrix $\hat{h}$.

Remark 2.3. The condition (ii) of Proposition 2.2 is equivalent to

$\operatorname{det} \hat{g}(x)=-g_{00}(t) \operatorname{det} \hat{h}(x)=-g_{00}(t) \sigma_{1}(\hat{h}) \sigma_{2}(\hat{h}) \sigma_{3}(\hat{h})=-g_{00}(t) e^{2 \int_{0}^{t} d t^{\prime} P\left(t^{\prime}\right)} \operatorname{det} \hat{h}_{0}(\vec{x})$, where $\operatorname{det} \hat{h}_{0}(\vec{x}) \in C^{\infty}(\Sigma)$ is a positive smooth function (the notation will become clear later).

The assertion follows from the combination of condition (ii) with the Laplace's formula,

$$
\frac{\partial}{\partial t} \operatorname{det} \hat{g}=\operatorname{det} \hat{g} \cdot \operatorname{Tr}\left[\hat{g}^{-1} \frac{\partial}{\partial t} \hat{g}\right]
$$

Because $\nabla$ is a metric connection, the restrictions of the previous proposition imply restrictions on the fiber metric $\langle,\rangle_{\mathfrak{g}}$. In case of a tensor bundle of rank $(m, n)$ with Levi-Civita connection, coefficients $\Gamma_{i}$ are expressed in Christoffel symbols and the fiber metric is induced by the spacetime metric, thus the restrictions fall onto the spacetime $(M, g)$.

Corollary 2.1. Let a local moving frame be chosen, such that the metric $\langle,\rangle_{\mathfrak{g}}$ is represented by the matrix $\hat{\mathfrak{g}}$. Conditions (iii) of Proposition 2.2 imply the following restrictions on $\hat{\mathfrak{g}}$ :

$$
\hat{\mathfrak{g}}(x)=\hat{\mathfrak{B}}^{T}(t) \hat{\mathfrak{g}}^{0}(\vec{x}) \hat{\mathfrak{B}}(t)
$$

where $\hat{\mathfrak{g}}^{0}$ and $\hat{\mathfrak{B}}$ are matrix valued smooth functions. In particular, for the a tensor bundle of rank $(m, n)$ to allow for seprataion it is necessary that the spacetime metric be represented by a matrix

$$
\hat{g}=1 \oplus\left(-\hat{h}_{0}(\vec{x}) \hat{B}(t)\right)
$$

where $\hat{h}_{0}$ and $\hat{B}$ are matrix valued smooth functions. 
Proof. Locally the conservation of the metric $\nabla\langle,\rangle_{\mathfrak{g}}=0$ can be written as

$$
\frac{\partial}{\partial x^{i}} \hat{\mathfrak{g}}-\Gamma_{i}^{T} \hat{\mathfrak{g}}-\hat{\mathfrak{g}} \Gamma_{i}=0,
$$

where $\Gamma_{i}$ are the matrices of the connection coefficients in the chosen frame. In particular, for $i=0$ we have

$$
\frac{\partial}{\partial t} \hat{\mathfrak{g}}(x)-\Gamma_{0}^{T}(t) \hat{\mathfrak{g}}(x)-\hat{\mathfrak{g}}(x) \Gamma_{0}(t)=0,
$$

where $\Gamma_{0}=\Gamma_{0}(t)$ was used. The solutions of this equation are of the form

$$
\hat{\mathfrak{g}}(x)=\hat{\mathfrak{B}}^{T}(t) \hat{\mathfrak{g}}^{0}(\vec{x}) \hat{\mathfrak{B}}(t),
$$

where

$$
\hat{\mathfrak{B}}(t)=e^{\int_{0}^{t} d t^{\prime} \Gamma_{0}\left(t^{\prime}\right)},
$$

and $\hat{\mathfrak{g}}^{0}(\vec{x})$ is a smooth symmetric matrix field on $\Sigma$.

Now if we identify the tensor space $\left(T_{p} M\right)_{n}^{m}$ with an $4^{n+m}$ dimensional vector space $V$ using a suitable bases, then each matrix $\Gamma_{i}$ will be a $4^{n+m-1} \times 4^{n+m-1}$ matrix of blocks, with blocks being the Christoffel symbols $\boldsymbol{\Gamma}_{i}$ for contravariant indices and $-\boldsymbol{\Gamma}_{i}^{T}$ for covariant indices. $\Gamma_{0}=\Gamma_{0}(t)$ means $\boldsymbol{\Gamma}_{0}=\boldsymbol{\Gamma}_{0}(t)$. With our time-separated metric we have

$$
\hat{g}=1 \oplus-\hat{h}
$$

One can find

$$
\boldsymbol{\Gamma}_{0}=0 \oplus\left(\frac{1}{2} \hat{h}^{-1} \frac{\partial \hat{h}}{\partial t}\right)=\boldsymbol{\Gamma}_{0}(t)=0 \oplus \hat{A}(t)
$$

for some smooth $3 \times 3$ matrix $\hat{A}(t)$. The solution is

$$
\hat{h}(x)=\hat{h}_{0}(\vec{x}) e^{2 \int_{0}^{t} d t^{\prime} \hat{A}\left(t^{\prime}\right)}=\hat{h}_{0}(\vec{x}) \hat{B}(t),
$$

for smooth symmetric commuting matrix fields $\hat{h}_{0}(\vec{x})$ and $\hat{B}(t)$.

Now the notation $\operatorname{det} \hat{h}_{0}$ of Remark 2.3 becomes clear, and we see that

$$
\operatorname{det} \hat{B}(t)=e^{2 \int_{0}^{t} d t^{\prime} P\left(t^{\prime}\right)}
$$

for a tensor bundle. Note that for the scalar field conditions (iii) are trivially satisfied and do not restrict the spacetime.

Remark 2.4. For the volume form measure $d \mu_{h}$ on $\Sigma_{t}$ we have locally

$$
d \mu_{h}(\vec{x})=\sqrt{\operatorname{det} \hat{h}(t, \vec{x})} d x^{1} d x^{2} d x^{3} .
$$

By Remark 2.3 we have

$$
\operatorname{det} \hat{h}(t, \vec{x})=e^{2 \int_{0}^{t} d t^{\prime} P\left(t^{\prime}\right)} \operatorname{det} \hat{h}_{0}(\vec{x}),
$$


hence

$$
d \mu_{h}(\vec{x})=e^{\int_{0}^{t} d t^{\prime} P\left(t^{\prime}\right)} d \mu_{h_{0}}(\vec{x})
$$

where

$$
d \mu_{h_{0}}(\vec{x})=\sqrt{\operatorname{det} \hat{h_{0}}(\vec{x})} d x^{1} d x^{2} d x^{3} .
$$

Henceforth by stating that a mode decomposition of $\operatorname{Sol}_{0}(\mathcal{T})$ exists we will mean that the assumptions of the Proposition 2.2 are satisfied and the corresponding covering is chosen. We are ready to formulate precisely the time dependent Fourier transform. Note that although $\zeta_{\alpha}$ are $t$-independent, the spatial metric $h$ and the fiber metric $\langle,\rangle_{\mathfrak{g}}$ depend on $t$, and $\zeta_{\alpha}$ are not orthonormal with respect to the measure $d \mu_{h}$ for all $t$ simultaneously. At this point we appoint once and forever to normalize $\zeta_{\alpha}$ such that they are orthonormal at $t=0$. Or equivalently, they are orthonormal with respect to the measure $d \mu_{h_{0}}$ of Remark 2.4 and the fiber metric $\mathfrak{g}^{0}$ of Corollary 2.1.

Definition 2.3. For $f \in \mathcal{D}(\mathcal{T})$ we define the time dependent Fourier transform $\tilde{f}(t, \alpha)=\mathcal{F}[f(t,)].(\alpha) \in C_{0}^{\infty}(\mathcal{I}, \tilde{\mathcal{D}}(\tilde{\Sigma}))$ by

$$
\mathcal{F}[f(t, .)](\alpha)=\int_{\Sigma_{t}} d \mu_{h_{0}}(\vec{x})\left\langle\bar{\zeta}_{\alpha}(\vec{x}), f(t, \vec{x})\right\rangle_{\mathfrak{g}^{0}} .
$$

Here we note another important corollary, which will be useful later. It will give the time dependent Plancherel formula.

Corollary 2.2. Suppose the assumptions of Proposition 2.2 are satisfied, and the corresponding covering is chosen. Then for all $f \in \mathcal{D}(\mathcal{T})$

$$
\left(\zeta_{\alpha}, f(t, .)\right)_{\Sigma_{t}}=I_{\alpha}(t) \mathcal{F}[f(t, .)](\alpha)
$$

and the time dependent Plancherel formula for the time-dependent Fourier transform is given by

$$
(f(t, .), h(t, .))_{\Sigma_{t}}=\int_{\tilde{\Sigma}} d \mu(\alpha) I_{\alpha}(t) \overline{\mathcal{F}[f(t, .)]}(\alpha) \mathcal{F}[h(t, .)](\alpha),
$$

where

$$
I_{\alpha}(t)=e^{\int_{0}^{t} d t^{\prime} F_{\alpha}\left(t^{\prime}\right)}
$$

Proof. By Remark 2.4

$$
\begin{gathered}
(f(t, .), h(t, .))_{\Sigma_{t}}=\int_{\Sigma_{t}} d \mu_{h}(\vec{x})(f(t, .), h(t, .))_{\mathfrak{g}}= \\
=e^{\int_{0}^{t} d t^{\prime} P\left(t^{\prime}\right)} \int_{\Sigma_{t}} d \mu_{h_{0}}(\vec{x})(f(t, .), h(t, .))_{\mathfrak{g}}
\end{gathered}
$$

At the same time by Corollary 2.1 we have

$$
(f(t, .), h(t, .))_{\mathfrak{g}}=(\hat{\mathfrak{B}}(t) f(t, .), \hat{\mathfrak{B}}(t) h(t, .))_{\mathfrak{g}^{0}} .
$$


Because we have normalized $\zeta_{\alpha}$ with respect to $d \mu_{h_{0}}$ and $\mathfrak{g}^{0}$, the conventional Plancherel formula holds for them,

$$
\int_{\Sigma_{t}} d \mu_{h_{0}}(\vec{x})(f(t, .), h(t, .))_{\mathfrak{g}^{0}}=\int_{\tilde{\Sigma}} d \mu(\alpha) \overline{\mathcal{F}[f(t, .)](\alpha)} \mathcal{F}[h(t, .)](\alpha) .
$$

Combining these three formulas we find

$$
(f(t, .), h(t, .))_{\Sigma_{t}}=e^{\int_{0}^{t} d t^{\prime} P\left(t^{\prime}\right)} \int_{\tilde{\Sigma}} d \mu(\alpha) \overline{\mathcal{F}[\hat{\mathfrak{B}}(t) f(t, .)](\alpha)} \mathcal{F}[\hat{\mathfrak{B}}(t) f(t, .)](\alpha) .
$$

Meanwhile

$$
\left(\zeta_{\alpha}, f(t, .)\right)_{\Sigma_{t}}=e^{\int_{0}^{t} d t^{\prime} P\left(t^{\prime}\right)} \int_{\Sigma_{t}} d \mu_{h_{0}}(\vec{x})\left(\hat{\mathfrak{B}}(t) \zeta_{\alpha}, \hat{\mathfrak{B}}(t) h(t, .)\right)_{\mathfrak{g}^{0}} .
$$

By definition

$$
\hat{\mathfrak{B}}(t) \zeta_{\alpha}=e^{\int_{0}^{t} d t^{\prime} \Gamma_{0}\left(t^{\prime}\right)} \zeta_{\alpha}=e^{\frac{1}{2} \int_{0}^{t} d t^{\prime}\left[A^{0}\left(t^{\prime}\right)-P\left(t^{\prime}\right)\right]} \zeta_{\alpha}=e^{\frac{1}{2} \int_{0}^{t} d t^{\prime}\left[F_{\alpha}\left(t^{\prime}\right)-P\left(t^{\prime}\right)\right]} \zeta_{\alpha},
$$

whence

$$
\left(\zeta_{\alpha}, f(t, .)\right)_{\Sigma_{t}}=e^{\frac{1}{2} \int_{0}^{t} d t^{\prime}\left[F_{\alpha}\left(t^{\prime}\right)+P\left(t^{\prime}\right)\right]} \mathcal{F}[\hat{\mathfrak{B}}(t) f(t, .)](\alpha) .
$$

Finally

$\hat{\mathfrak{B}}(t) f(t, \vec{x})=\int_{\tilde{\Sigma}} d \mu(\alpha) \mathcal{F}[f(t,)].(\alpha) \hat{\mathfrak{B}}(t) \zeta_{\alpha}(\vec{x})=\int_{\tilde{\Sigma}} d \mu(\alpha) \mathcal{F}[f(t,)].(\alpha) e^{\frac{1}{2} \int_{0}^{t} d t^{\prime}\left[F_{\alpha}\left(t^{\prime}\right)-P\left(t^{\prime}\right)\right]} \zeta_{\alpha}$,

thus

$$
\mathcal{F}[\hat{\mathfrak{B}}(t) f(t, .)](\alpha)=e^{\frac{1}{2} \int_{0}^{t} d t^{\prime}\left[F_{\alpha}\left(t^{\prime}\right)-P\left(t^{\prime}\right)\right]} \mathcal{F}[f(t, .)](\alpha) .
$$

The assertions now easily follow.

At last we compute the spectra of operators $A^{0}$ and $B^{0}$ for the tensor bundle to find the functions $F_{\alpha}$ and $H_{\alpha}$. In view of Corollary 2.1 the function $P(t)$ becomes

$$
P(t)=\frac{1}{2} \operatorname{Tr}\left[\hat{B}^{-1}(t) \frac{\partial \hat{B}}{\partial t}(t)\right] .
$$

Then

$$
\begin{gathered}
\operatorname{Spec}\left\{A^{0}\right\}=2 \operatorname{Spec}\left\{\Gamma_{0}\right\}+P(t), \\
\operatorname{Spec}\left\{B^{0}\right\}=\left\{\dot{\sigma}(t)+\sigma^{2}(t)+\sigma(t) P(t): \sigma \in \operatorname{Spec}\left\{\Gamma_{0}\right\}\right\} .
\end{gathered}
$$

As a useful example we calculate these spectra for the scalar and 1-form fields on uniformly expanding (e.g., FRW) manifolds,

$$
d s^{2}=d t^{2}-a^{2}(t) d \sigma^{2}(\vec{x}) .
$$

Here the matrix $\hat{B}(t)=a^{2}(t) 1$, and hence

$$
\hat{A}(t)=\frac{\partial}{\partial t} \ln a(t) 1=H(t) 1,
$$


and

$$
P(t)=3 H(t), H(t)=\frac{\dot{a}(t)}{a(t)} .
$$

For scalar case $n=m=0$ and we have

$$
\operatorname{Spec}\left\{\Gamma_{0}\right\}=\{0\},
$$

thus

$$
\operatorname{Spec}\left\{A^{0}\right\}=\{3 H(t)\}, \operatorname{Spec}\left\{B^{0}\right\}=\{0\},
$$

as well known. For the 1 -form case, $m=0, n=1$, we have

$$
\operatorname{Spec}\left\{\Gamma_{0}\right\}=\{0,-H(t)\} \text {, }
$$

and thereby

$$
\operatorname{Spec}\left\{A^{0}\right\}=\{3 H(t), H(t)\}, \operatorname{Spec}\left\{B^{0}\right\}=\left\{0,-\dot{H}(t)-2 H^{2}(t)\right\},
$$

where the first members are similar to the scalar case and represent the scalar modes, but second ones represent the transversal and longitudinal modes.

As we have seen, for the separation it is necessary that the evolution of the metric be represented by linear transformations. If the connection also satisfies such a condition in a suitable sense, than the operator $D_{\Sigma_{t}}$ is essentially the same at every $t$ up to some scale factors. (Maybe the condition (iii) of the main proposition already implies such a restriction on the connection, but we are not sure yet.) This will be the case for all our bundles of interest, and it will provide analytical advantages. To summarize what we expect precisely we give the following definitions.

Definition 2.4. We will say that the operator $D_{\Sigma_{t}}$ has a strictly uniform spectrum over time if there exists a lower semi-bounded function $\omega(\alpha)$ on $\tilde{\Sigma}$, a positive smooth function $C(t)>0$ and a smooth function $\tilde{m}^{\star}(t)$ such that $\lambda_{\alpha}(t)=\omega(\alpha) C(t)+\tilde{m}^{\star}(t)$, or equivalently, the expression

$$
\frac{d}{d t} \ln \left|\lambda_{\alpha}(t)-\tilde{m}^{\star}(t)\right|
$$

does not depend on $\alpha$.

This is a rather strong condition. It basically requires that the eigenspaces of $D_{\Sigma_{t}}$ coincide for different $t$ up to an overall shift, and that eigenvalues be linearly proportional. Such a property would be very comfortable, but it does not hold for some models of our interest. In particular, it does not hold for the Bianchi I model with distortions. Hence we will derive some of our results under a milder restriction which holds at least in all cosmological situations where the spectral theory is explicit enough so that it can be checked. (The explicit spectral theory of the scalar field on Bianchi I-VII spacetimes will appear in a subsequent publication.)

Definition 2.5. We will say that the operator $D_{\Sigma_{t}}$ has a loosely uniform spectrum over time if

$$
\left|\frac{d}{d t} \ln \right| \lambda_{\alpha}(t)-\tilde{m}^{\star}(t)|| \leq C_{\mathcal{R}}, \forall t \in \mathcal{R}, \alpha \in \tilde{\Sigma}
$$


for any compact interval $\mathcal{R} \subset \mathcal{I}$, and for some $0<C_{\mathcal{R}} \in \mathbb{R}$ and a smooth function $\tilde{m}^{\star}(t)$.

If the Fourier transform is conventional, then it will be natural to require that $\omega$ be an analytic function on $\tilde{\Sigma}$.

\subsection{Some properties of the mode solutions}

In this section we investigate the equation $(\mathrm{Eq} 2.12)$ and obtain some useful properties of the mode solutions $T_{\alpha}$. The mode equation is

$$
\ddot{T}_{\alpha}(t)+F_{\alpha}(t) \dot{T}_{\alpha}(t)+G_{\alpha}(t) T_{\alpha}(t)=0,
$$

where

$$
G_{\alpha}(t)=H_{\alpha}(t)+\lambda_{\alpha}(t),
$$

and $\lambda_{\alpha}(t)$ are the eigenvalues of the operator $D_{\Sigma_{t}}=-\Delta_{t}+m^{\star}(x)$. Note that $G_{\alpha}$ may become null or negative for some rates of expansion. This corresponds to the socalled positive back-reaction in a linear system and results in exponential solutions. This is an interesting phenomenon appearing in non scalar fields (for scalar fields $H_{\alpha}=0$ ), and its significance is not yet completely clear to us. To understand it one could, for instance, track its influence on the energy-momentum tensor etc. It is not obvious that this is really a physical infrared instability, because it may occur for the co-vector field but not for the vector counterpart, for instance. It is also worth mentioning, that for the co-vector (1-form) field the introduction of a conformal coupling precisely cancels this instability. It seems plausible that for each field there is a choice of the coupling constant which compensates this bad infrared behavior. We say infrared, because $\lambda_{\alpha}(t)$ attains arbitrarily large positive values at any $t$, thus on an unbounded subbundle of $R \times \tilde{\Sigma}, G_{\alpha}$ is positive.

We will make this more explicit under the assumption, that $D_{\Sigma_{t}}$ has a strictly uniform spectrum. Then the function $C(t)$ is uniformly bounded from below, and the functions $H_{\alpha}$ and $\tilde{m}^{\star}$ are uniformly bounded from above on any compact interval $\mathcal{R}$. On the other hand $\omega \rightarrow+\infty$, hence is suffices to choose $\omega$ large enough to make $G_{\alpha}=H_{\alpha}+C \omega+\tilde{m}^{\star}>0$.

Fix a component $\tilde{\Sigma}^{i}$ and write $H=H_{\alpha}, F=F_{\alpha}$ and $I=I_{\alpha}$ for all $\alpha \in \tilde{\Sigma}^{i}$. Define a new variable

$$
s(t)=\int_{0}^{t} d \tau e^{-\int_{0}^{\tau} d \tau^{\prime} F\left(\tau^{\prime}\right)}=\int_{0}^{t} d \tau I^{-1}(\tau),
$$

which is in a smooth monotone bijective correspondence with $t$. The inverse function will be denoted by $t(s)$. Regarding all the acting functions of $t$ as functions of $s$ we obtain

$$
\ddot{T}_{\alpha}(s)+\Lambda_{\alpha}(s) T_{\alpha}(s)=0
$$

where

$$
\Lambda_{\alpha}(s)=\left[G_{\alpha}(t) e^{2 \int_{0}^{t} d \tau F(\tau)}\right]_{t=t(s)}=G_{\alpha}(s) I^{2}(s) .
$$


This is a time dependent harmonic oscillator equation, to which the results in the appendix apply.

Remark 2.5. Note that the Wronski determinant of two solutions $Q, R$

$$
\operatorname{det} W[Q, R](s)=Q(s) \dot{R}(s)-\dot{Q}(s) R(s)=\mathrm{const}
$$

in variable $t$ becomes

$\operatorname{det} W[Q, R](t)=\frac{d t}{d s}(Q(t) \dot{R}(t)-\dot{Q}(t) R(t))=I(t)(Q(t) \dot{R}(t)-\dot{Q}(t) R(t))=$ const.

Applying Corollary Appendix B.1 to (Eq 2.16) for different $\alpha$ we find estimates which in principle depend on $\alpha$ in a complicated way. But under the assumption of loose uniformity on $D_{\Sigma_{t}}$ we will be able to invoke more comfortable expressions.

Proposition 2.3. Suppose $D_{\Sigma_{t}}$ has a loosely uniform spectrum over time. Then for a family of arbitrary solutions $T_{\alpha}$ of (Eq2.16) the following estimate holds

$$
\left|T_{\alpha}(s)\right| \leq R_{\mathcal{R}}\left|T_{\alpha}(0)\right|+\frac{S_{\mathcal{R}}}{\max \left\{1, \sqrt{U_{\mathcal{R}}+T_{\mathcal{R}} \lambda_{\alpha}(0)}\right\}}\left|\dot{T}_{\alpha}(0)\right|, \forall s \in \mathcal{R},
$$

with $0<R_{\mathcal{R}}, S_{\mathcal{R}}, T_{\mathcal{R}} \in \mathbb{R}$ and $U_{\mathcal{R}} \in \mathbb{R}$, for any compact interval $\mathcal{R}$.

Proof. Fix a compact interval $\mathcal{R}$ and for each $\alpha \in \tilde{\Sigma}^{i}$ apply Corollary Appendix B.1 with $\Lambda_{\alpha}(s)=I^{2}(s) H(s)+I^{2}(s) \lambda_{\alpha}(s)$. Because $\Lambda_{\alpha}$ is real, we get $A_{\mathcal{R}}(\alpha)=0$. As $\lambda_{\alpha}(s)$ is lower semi-bounded we have

$$
p_{\mathcal{R}} \doteq \inf _{\tilde{\Sigma}} \inf _{\mathcal{R}} \lambda_{\alpha}>-\infty .
$$

Denote $m_{\mathcal{R}}=\inf _{\mathcal{R}}\left\{I^{2} H\right\}$ and $n_{\mathcal{R}}=\inf _{\mathcal{R}}\left\{I^{2}\right\}>0$. Then

$$
c_{\mathcal{R}}(\alpha) \geq m_{\mathcal{R}}+n_{\mathcal{R}} \inf _{\mathcal{R}} \lambda_{\alpha} \geq m_{\mathcal{R}}+n_{\mathcal{R}} p_{\mathcal{R}}
$$

It follows that $\kappa(\alpha) \leq \sqrt{1+\left|m_{\mathcal{R}}+n_{\mathcal{R}} p_{\mathcal{R}}\right|}$ and $e_{\mathcal{R}}(\alpha) \geq 1+\max \left\{0, m_{\mathcal{R}}+\right.$ $\left.n_{\mathcal{R}} \inf _{\mathcal{R}} \lambda_{\alpha}\right\}$. Denote $M_{\mathcal{R}}=\sup _{\mathcal{R}}\left\{\left|I^{2} H\right|\right\} \geq 0$ and $N_{\mathcal{R}}=\sup _{\mathcal{R}}\left\{I^{2}\right\}>0$. We find next

$$
D_{\mathcal{R}}(\alpha) \leq 1+\left|m_{\mathcal{R}}+n_{\mathcal{R}} p_{\mathcal{R}}\right|+M_{\mathcal{R}}+N_{\mathcal{R}}\left|\sup _{\mathcal{R}} \lambda_{\alpha}\right| .
$$

Now we observe that by loose uniformity

$$
\left|\ln \frac{\left|\lambda_{\alpha}(s)-\tilde{m}^{\star}(s)\right|}{\left|\lambda_{\alpha}\left(s^{\prime}\right)-\tilde{m}^{\star}\left(s^{\prime}\right)\right|}\right|=\left|\int_{s}^{s^{\prime}} d \sigma \partial_{s} \ln \right| \lambda_{\alpha}(\sigma)-\tilde{m}^{\star}(\sigma)|| \leq|\mathcal{R}| \sqrt{N_{\mathcal{R}}} C_{\mathcal{R}},
$$

hence

$$
\begin{gathered}
\sup _{\mathcal{R}}\left|\lambda_{\alpha}-\tilde{m}^{\star}\right| \leq\left|\lambda_{\alpha}(0)-\tilde{m}^{\star}(0)\right| e^{|\mathcal{R}| \sqrt{N_{\mathcal{R}}} C_{\mathcal{R}}} \\
\inf _{\mathcal{R}}\left|\lambda_{\alpha}-\tilde{m}^{\star}\right| \geq\left|\lambda_{\alpha}(0)-\tilde{m}^{\star}(0)\right| e^{-|\mathcal{R}| \sqrt{N_{\mathcal{R}}} C_{\mathcal{R}}} .
\end{gathered}
$$


Note that whenever $\lambda_{\alpha}(0)-\tilde{m}^{\star}(0)>0$ then it follows by continuity that $\lambda_{\alpha}(s)-$ $\tilde{m}^{\star}(s)>0$ for all $s \in \mathcal{R}$. Denote

$$
\lambda_{\text {min }}=\tilde{m}^{\star}(0)-\min \left\{0, \inf _{\mathcal{R}} \tilde{m}^{\star} \cdot e^{|\mathcal{R}| \sqrt{N_{\mathcal{R}}} C_{\mathcal{R}}}\right\}-\min \left\{0, \frac{m_{\mathcal{R}}}{n_{\mathcal{R}}} e^{|\mathcal{R}| \sqrt{N_{\mathcal{R}}} C_{\mathcal{R}}}\right\} .
$$

Then from $\lambda_{\alpha}(0)>\lambda_{\text {min }}$ it follows $\lambda_{\alpha}(0)-\tilde{m}^{\star}(0)>0, m_{\mathcal{R}}+n_{\mathcal{R}} \inf _{\mathcal{R}} \lambda_{\alpha}>0$ and

$$
\inf _{\mathcal{R}} \lambda_{\alpha} \geq \inf _{\mathcal{R}} \tilde{m}^{\star}+\left(\lambda_{\alpha}(0)-\tilde{m}^{\star}(0)\right) e^{-|\mathcal{R}| \sqrt{N_{\mathcal{R}}} C_{\mathcal{R}}}>0 .
$$

Now we have that

$e_{\mathcal{R}}(\alpha) \geq 1+\chi\left[\lambda_{\alpha}(0)>\lambda_{\text {min }}\right]\left(m_{\mathcal{R}}+n_{\mathcal{R}}\left(\inf _{\mathcal{R}} \tilde{m}^{\star}+\left(\lambda_{\alpha}(0)-\tilde{m}^{\star}(0)\right) e^{-|\mathcal{R}| \sqrt{N_{\mathcal{R}}} C_{\mathcal{R}}}\right)\right)$,

where the characteristic function $\chi$ plays here the role of the condition checking. From (Eq2.17) we find

$$
\sup _{\mathcal{R}}\left|\lambda_{\alpha}\right| \leq \sup _{\mathcal{R}} \tilde{m}^{\star}+\left|\lambda_{\alpha}(0)-\tilde{m}^{\star}(0)\right| e^{|\mathcal{R}| \sqrt{N_{\mathcal{R}}} C_{\mathcal{R}}}
$$

whence

$$
D_{\mathcal{R}}(\alpha) \leq 1+\left|m_{\mathcal{R}}+n_{\mathcal{R}} p_{\mathcal{R}}\right|+M_{\mathcal{R}}+N_{\mathcal{R}}\left(\sup _{\mathcal{R}} \tilde{m}^{\star}+\left|\lambda_{\alpha}(0)-\tilde{m}^{\star}(0)\right| e^{|\mathcal{R}| \sqrt{N_{\mathcal{R}}} C_{\mathcal{R}}}\right) .
$$

Thus we establish that for $\lambda_{\alpha}(0) \leq \lambda_{\min }$

$\frac{D_{\mathcal{R}}(\alpha)}{e_{\mathcal{R}}(\alpha)} \leq 1+\left|m_{\mathcal{R}}+n_{\mathcal{R}} p_{\mathcal{R}}\right|+M_{\mathcal{R}}+N_{\mathcal{R}}\left(\sup _{\mathcal{R}} \tilde{m}^{\star}+\left(\left|\lambda_{m i n}\right|+\left|\tilde{m}^{\star}(0)\right|\right) e^{|\mathcal{R}| \sqrt{N_{\mathcal{R}}} C_{\mathcal{R}}}\right)$,

and for $\lambda_{\alpha}(0)>\lambda_{\min }$

$$
\begin{aligned}
\frac{D_{\mathcal{R}}(\alpha)}{e_{\mathcal{R}}(\alpha)} \leq e^{2|\mathcal{R}| \sqrt{N_{\mathcal{R}}} C_{\mathcal{R}}} N_{\mathcal{R}}\left(\frac{1}{n_{\mathcal{R}}}+\frac{1+\left|m_{\mathcal{R}}+n_{\mathcal{R}} p_{\mathcal{R}}\right|+M_{\mathcal{R}}+N_{\mathcal{R}}\left|\sup _{\mathcal{R}} \tilde{m}^{\star}\right|}{N_{\mathcal{R}} e^{|\mathcal{R}| \sqrt{N_{\mathcal{R}}} C_{\mathcal{R}}}}+\right. \\
\left.+\frac{1+\left|m_{\mathcal{R}}\right|+n_{\mathcal{R}}\left|\inf _{\mathcal{R}} \tilde{m}^{\star}\right|}{n_{\mathcal{R}} e^{-|\mathcal{R}| \sqrt{N_{\mathcal{R}}} C_{\mathcal{R}}}}\right)
\end{aligned}
$$

Finally

$\frac{d}{d s} \ln \left(\kappa^{2}+\Lambda_{\alpha}\right)=\frac{\frac{d}{d s}\left(I^{2}\left(H+\tilde{m}^{\star}\right)\right)+\frac{d}{d s}\left(I^{2}\right)\left(\lambda_{\alpha}-\tilde{m}^{\star}\right)+I^{2}\left(\lambda_{\alpha}-\tilde{m}^{\star}\right) \frac{d}{d s} \ln \left|\lambda_{\alpha}-\tilde{m}^{\star}\right|}{\kappa^{2}+I^{2} H+I^{2} \lambda_{\alpha}}$.

Denote $P_{\mathcal{R}}=\sup _{\mathcal{R}}\left|\partial_{s}\left(I^{2}\left(H-\tilde{m}^{\star}\right)\right)\right| \geq 0$ and $Q_{\mathcal{R}}=\sup _{\mathcal{R}}\left|\partial_{s}\left(I^{2}\right)\right| \geq 0$. Again using the loose uniformity, for $\lambda_{\alpha}-\tilde{m}^{\star} \leq 1$

$$
\left|\frac{d}{d s} \ln \left(\kappa^{2}+\Lambda_{\alpha}\right)\right| \leq P_{\mathcal{R}}+Q_{\mathcal{R}}+\left(N_{\mathcal{R}}\right)^{\frac{3}{2}} C_{\mathcal{R}}
$$

and else

$$
\left|\frac{d}{d s} \ln \left(\kappa^{2}+\Lambda_{\alpha}\right)\right| \leq \frac{P_{\mathcal{R}}+Q_{\mathcal{R}}+\left(N_{\mathcal{R}}\right)^{\frac{3}{2}} C_{\mathcal{R}}}{n_{\mathcal{R}}} .
$$


Summarizing this all we find that by Corollary Appendix B.1 there exist numbers $0<R_{\mathcal{R}}, S_{\mathcal{R}}, T_{\mathcal{R}} \in \mathbb{R}$ and $U_{\mathcal{R}} \in \mathbb{R}$ such that for a family of arbitrary solutions $T_{\alpha}$ we have

$$
\left|T_{\alpha}(s)\right| \leq R_{\mathcal{R}}\left|T_{\alpha}(0)\right|+\frac{S_{\mathcal{R}}}{\max \left\{1, \sqrt{U_{\mathcal{R}}+T_{\mathcal{R}} \lambda_{\alpha}(0)}\right\}}\left|\dot{T}_{\alpha}(0)\right|,
$$

what was to be proven.

The result can be strengthened under additional assumptions. These are perhaps too restrictive, but they appear to be sufficient for some important applications. Let $\mathbb{H}_{a}=\{z \in \mathbb{C}:|\Im z|<a\}$.

Proposition 2.4. Suppose the bundle $\mathcal{T}$ is analytic, so that all functions figuring in (Eq2.16) are real analytic functions of $s$. Suppose further that $D_{\Sigma_{t}}$ has a strictly uniform spectrum. Choose the initial data to be $T_{\alpha}(0)=p(\omega(\alpha))$ and $\dot{T}_{\alpha}(0)=$ $q(\omega(\alpha))$, where $p(\omega), q(\omega)$ are holomorphic functions on $\mathbb{H}_{a}$ for some $a>0$. Then for each $s, T_{\alpha}(s)=r_{s}(\omega(\alpha))$, where $r_{s}(\omega)$ is holomorphic in $\omega$ on $\mathbb{H}_{a}$ and real analytic in $s$, and for any compact interval $\mathcal{R}$ it holds

$$
\left|r_{s}(\omega)\right| \leq R_{\mathcal{R}}|p(\omega)|+\frac{S_{\mathcal{R}}}{\max \left\{1, \sqrt{U_{\mathcal{R}}+T_{\mathcal{R}} \Re \omega}\right\}}|q(\omega)|, \forall s \in \mathcal{R},
$$

with $0<R_{\mathcal{R}}, S_{\mathcal{R}}, T_{\mathcal{R}} \in \mathbb{R}$ and $U_{\mathcal{R}} \in \mathbb{R}$.

Proof. By strict uniformity we have $\lambda_{\alpha}(t)=\omega(\alpha) C(t)+\tilde{m}^{\star}(t)$, and if the initial data depend only on $\omega$, then the solutions will also be such. Therefore for convenience we write

$$
\ddot{T}_{\omega}(s)+I^{2}(s)\left(H(s)+\omega C(s)+\tilde{m}^{\star}(s)\right) T_{\omega}(s)=0
$$

with $T_{\omega}(0)=p(\omega)$ and $\dot{T}_{\omega}(0)=q(\omega)$. From the theory of power series it is clear that any real analytic function on $s(\mathcal{I})$ can be extended to a holomorphic function in some open neighborhood $\delta(s(\mathcal{I}))$ of $s(\mathcal{I})$. Consider $(\mathrm{Eq} 2.18)$ as a complex differential equation, then for any $\omega \in \mathbb{H}_{a}$, by Satz 4.1 and Satz 4.2 of [16] there exist neighborhoods $\delta(0)$ of 0 and $\delta(\omega)$ of $\omega$ such that $T_{\omega}(s)$ is holomorphic in $\delta(0) \times \delta(\omega)$. At the same time by Satz 5.3 of [16], for any $\omega \in \mathbb{H}_{a}$ the solution $T_{\omega}$ can be analytically continued to the whole of $\delta(s(\mathcal{I}))$. Thus $T_{\omega}(s)$ is holomorphic in $\delta(\mathbb{R}) \times \mathbb{H}_{a}$. Now restrict back to the real axis and fix the interval $\mathcal{R}$. The reasoning of the previous proposition can be repeated literally except that now $A_{\mathcal{R}}(\alpha)$ is not zero but equals $A_{\mathcal{R}}(\omega)=|\Im \omega| \sup _{\mathcal{R}}\left\{I^{2} C\right\}<a \sup _{\mathcal{R}}\left\{I^{2} C\right\}$. This results in a similar formula as in Proposition 2.3 with perhaps different coefficients, and that proves our assertion.

We have an immediate corollary.

Corollary 2.3. Under the assumptions of Proposition 2.4 if $p, q \in \mathcal{A}\left(\mathbb{H}_{a}\right)$ then for each $s \in \mathcal{R}, r_{s} \in \mathcal{A}\left(\mathbb{H}_{a}\right)$. 


\subsection{Mode decomposition of weak solutions}

The aim of this section will be to extend the mode decomposition of $\operatorname{Sol}_{0}(\mathcal{T})$ obtained previously to entire $\mathcal{D}(\mathcal{T})_{0}^{\prime}$. Here we assume all the conditions of Proposition 2.2 are satisfied, and we have chosen the system $\{u, v\}_{\alpha}$ with $u_{\alpha}=T_{\alpha} \zeta_{\alpha}$ and $v_{\alpha}=\bar{T}_{\alpha} \zeta_{\alpha}$, which span $\operatorname{Sol}_{0}(\mathcal{T})$. For convenience we will also assume at least the part (iv) of the definition of the conventional Fourier transform to hold.

Unfortunately we do not have a precise analytical description of the Fourier transformed test function space $\tilde{\mathcal{D}}(\tilde{\Sigma})$ even under the assumptions of the conventional Fourier transform, as it was, for instance, in the Euclidean space by PaleyWiener theorem. In particular we need to know for which modes $T_{\alpha}$ it holds

$$
T_{\alpha}(t) \tilde{f}(\alpha) \in \tilde{\mathcal{D}}(\tilde{\Sigma}), \forall \tilde{f}(\alpha) \in \tilde{\mathcal{D}}(\tilde{\Sigma}), t \in \mathcal{I} .
$$

At least we are able to find a sufficient condition under additional assumptions.

Proposition 2.5. Suppose the bundle $\mathcal{T}$ is analytic and $D_{\Sigma_{t}}$ has a strictly uniform spectrum. For each $\alpha \in \tilde{\Sigma}^{i}$ set $T_{\alpha}(0)=p^{i}(\omega(\alpha))$ and $\dot{T}_{\alpha}(0)=q^{i}(\omega(\alpha))$, where $p^{i}, q^{i} \in \mathcal{A}\left[\mathbb{H}_{0}\right]$. Then (Eq[2.19) holds.

Proof. Choose the interval $\mathcal{R}$ such that it contains both 0 and $t$. First we note that by Corollary 2.3 for $\alpha \in \tilde{\Sigma}^{i}$ we have $T_{\alpha}(t)=r_{t}^{i}(\omega(\alpha))$ with $r_{t}^{i} \in \mathcal{A}\left[\mathbb{H}_{0}\right]$. Denote $F_{t}^{i}(\lambda)=r_{t}^{i}\left(\frac{\lambda}{C(t)}\right) \in \mathcal{A}\left[\mathbb{H}_{0}\right]$. Obviously for any $f \in \mathcal{D}\left(\mathcal{T}_{t}\right)$,

$$
F_{t}^{i}\left(\lambda_{\alpha}(t)\right) \tilde{f}(\alpha)=\left[\widetilde{F_{t}^{i}\left(D_{\Sigma_{t}}\right)} f\right](\alpha),
$$

where $F_{t}^{i}\left(D_{\Sigma_{t}}\right)$ is defined by functional calculus. Then by Proposition Appendix C.1

$$
F_{t}^{i}\left(\lambda_{\alpha}(t)\right) \tilde{f}(\alpha) \in \tilde{\mathcal{D}}(\tilde{\Sigma})
$$

Let $\left\{U_{n}\right\}$ be a covering by local trivializations of $\mathcal{T}_{t}$, and let $\left\{\imath_{n}\right\}$ be a subordinate partition of unity. The support of $f$ is covered by $N_{f}$ (finite) trivializing neighborhoods, and we write $f=\sum_{n} \imath_{n} f=\sum_{n} f_{n}$. It follows $\tilde{f}=\sum_{n} \tilde{f}_{n}$ and $T_{\alpha}(t) \tilde{f}(\alpha)=\sum_{n} T_{\alpha}(t) \tilde{f}_{n}(\alpha)$. Consider $f_{n}$ as a section in the trivial bundle $\pi^{-1}\left(U_{n}\right)$. As we have seen already (and as we will see even more evidently for homogeneous spacetimes in the next chapter) each component $\tilde{\Sigma}^{i}$ supports the Fourier transform of one fiber component in some local frame. Thus we can write $f_{n}=\sum_{i} f_{n}^{i}$, where $f_{n}^{i} \in \mathcal{D}\left(U_{n}\right)$ and $\tilde{f}_{n}^{i}$ is supported in $\tilde{\Sigma}^{i}$. We get

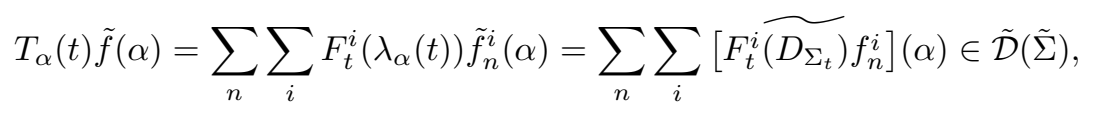

which completes the proof.

Remark 2.6. An argument involving local trivializations as in the proof of Proposition 2.5 will show that the multiplication of $\mathcal{F}[f(t,)$.$] by I_{\alpha}(t)$ amounts to multiplication of each fiber component by a number, hence $I_{\alpha}(t) \mathcal{F}[f(t,).] \in \tilde{\mathcal{D}}(\tilde{\Sigma})$ for all $t \in \mathcal{I}$. 
Two useful facts about the time dependent Fourier transform can be given by the following

Proposition 2.6. Let $\tilde{f}(t, \alpha) \in C_{0}^{\infty}(\mathcal{I}, \tilde{\mathcal{D}}(\tilde{\Sigma}))$. then

(i) $f(t, \vec{x})=\mathcal{F}^{-1}[\tilde{f}(t, \alpha)] \in \mathcal{D}(\mathcal{T})$

(ii) $\int_{\mathcal{I}} d t \tilde{f}(t, \alpha) \in \tilde{\mathcal{D}}(\tilde{\Sigma})$.

Proof. Let

$$
f(t, \vec{x})=\mathcal{F}^{-1}[\tilde{f}(t, \alpha)]=\int_{\tilde{\Sigma}} d \mu(\alpha) \tilde{f}(t, \alpha) \zeta_{\alpha}(\vec{x}) .
$$

For each $t \in \mathcal{I}$ we have $\tilde{f}(t, \alpha) \in \tilde{\mathcal{D}}(\tilde{\Sigma})$ and therefore $f(t, \vec{x}) \in \mathcal{D}\left(\mathcal{T}_{t}\right)$. If the compact interval $A \subset \mathcal{I}$ is such that $\forall t \notin A, \tilde{f}(t, \alpha)=0$, then obviously $\forall t \notin A, f(t, \vec{x})=0$. Because the integration converges in $L^{2}(\tilde{\Sigma}, \mu)$, differentiation can be interchanged with the integral, thus $f(t, \vec{x})$ is smooth in $t$. The part (i) is proven.

Now write

$$
\tilde{f}(t, \alpha)=\int_{\Sigma_{t}} d \mu_{h_{0}}(\vec{x})\left\langle\bar{\zeta}_{\alpha}(\vec{x}), f(t, \vec{x})\right\rangle_{\mathfrak{g}^{0}}
$$

and

$$
\begin{aligned}
& \int_{\mathcal{I}} d t \tilde{f}(t, \alpha)=\int_{\mathcal{I}} d t \int_{\Sigma_{t}} d \mu_{h_{0}}(\vec{x})\left\langle\bar{\zeta}_{\alpha}(\vec{x}), f(t, \vec{x})\right\rangle_{\mathfrak{g}^{0}}= \\
= & \int_{\Sigma_{t}} d \mu_{h_{0}}(\vec{x})\left\langle\bar{\zeta}_{\alpha}(\vec{x}), \int_{\mathcal{I}} d t f(t, \vec{x})\right\rangle_{\mathfrak{g}^{0}}=\mathcal{F}\left[\int_{\mathcal{I}} d t f(t, \vec{x})\right],
\end{aligned}
$$

where Fubini's theorem was used with the justification that both integrals run over compact supports [17]. For the part (ii) it remains to show that $\int_{\mathcal{I}} d t f(t, \vec{x}) \in \mathcal{D}\left(\mathcal{T}_{t}\right)$. But this is again clear because the integral runs over a compact support.

Next we want to show that the Cauchy problem can be well-posed in the distributional sense. We will do it by generalizing (Eq2.1) to distributional solutions.

Proposition 2.7. For any $u_{0}, u_{1} \in \mathcal{D}\left(\mathcal{T}_{t}\right)^{\prime}$ there exists a unique $\jmath\left(u_{0}, u_{1}\right)=u \in$ $\mathcal{D}(\mathcal{T})_{0}^{\prime}$ such that

$$
u(f)=u_{0}\left(i_{t}^{*}\left(\nabla_{t} E[f]\right)\right)-u_{1}\left(i_{t}^{*}(E[f])\right), \forall f \in \mathcal{D}(\mathcal{T}) .
$$

Proof. By Proposition 2.1 we know that $E$ is surjective, so we denote the bijective part of $E$ to be $E_{\downarrow}: \mathcal{D}(\mathcal{T}) / \operatorname{ker} E \rightarrow \operatorname{Sol}_{0}(\mathcal{T})$. For surjectivity of $\jmath$ it suffices to set

$$
u_{0}\left(v_{1}\right)=u\left(E_{\uparrow}^{-1}\left[\jmath\left(0, v_{1}\right)\right]\right), u_{1}\left(v_{0}\right)=-u\left(E_{\uparrow}^{-1}\left[\jmath\left(v_{0}, 0\right)\right]\right), \forall v_{0}, v_{1} \in \mathcal{D}\left(\mathcal{T}_{t}\right) .
$$

Indeed,

$$
u(f)=u\left(E_{\uparrow}^{-1}\left[E_{\uparrow}[f]\right]\right)=u\left(E_{\uparrow}^{-1}\left[\jmath\left(i_{t}^{*}\left(E_{\uparrow}[f]\right), i_{t}^{*}\left(\nabla_{t} E_{\uparrow}[f]\right)\right)\right]\right)=
$$


$=u\left(E_{\uparrow}^{-1}\left[\jmath\left(i_{t} *\left(E_{\uparrow}[f]\right), 0\right)\right]\right)+u\left(E_{\uparrow}^{-1}\left[\jmath\left(0, i_{t}^{*}\left(\nabla_{t} E_{\uparrow}[f]\right)\right)\right]\right)=u_{0}\left(i_{t}^{*}\left(\nabla_{t} E[f]\right)\right)-u_{1}\left(i_{t}^{*}(E[f])\right)$.

For injectivity of $\jmath$ let $u_{0}, u_{1} \in \mathcal{D}\left(\mathcal{T}_{t}\right)^{\prime}$ be given. Define $u$ as in the statement. Then obviously $u(D f)=0$ because $E D f=0$ for any $f \in \mathcal{D}(\mathcal{T})$, hence $u \in \mathcal{D}\left(\mathcal{T}_{t}\right)_{0}^{\prime}$. Now suppose the same formula holds also for different $u_{0}^{\prime}, u_{1}^{\prime} \in \mathcal{D}\left(\mathcal{T}_{t}\right)^{\prime}$ with the same $u$. Then we have

$$
0=\left(u_{0}-u_{0}^{\prime}\right)\left(i_{t}^{*}\left(\nabla_{t} E[f]\right)\right)-\left(u_{1}-u_{1}^{\prime}\right)\left(i_{t}^{*}(E[f])\right), \forall f \in \mathcal{D}(\mathcal{T}) .
$$

Evaluating on $f=E_{\uparrow}^{-1}\left(\jmath\left(v_{0}, 0\right)\right)$ and $g=E_{\uparrow}^{-1}\left(\jmath\left(0, v_{1}\right)\right)$ for arbitrary $v_{0}, v_{1} \in \mathcal{D}\left(\mathcal{T}_{t}\right)$ we find $u_{0}=u_{0}^{\prime}$ and $u_{1}=u_{1}^{\prime}$.

Now we come to the main assertion. Let the modes $T_{\alpha}$ be chosen such that (Eq2.19) holds.

Proposition 2.8. Under the assumptions made, there exist closed topological subspaces $\tilde{\mathcal{D}}^{u}(\tilde{\Sigma}), \tilde{\mathcal{D}}^{v}(\tilde{\Sigma}) \subset \tilde{\mathcal{D}}(\tilde{\Sigma})$, such that for any $\psi \in \mathcal{D}(\mathcal{T})_{0}^{\prime}$ there are unique distributions $a^{\psi} \in \tilde{\mathcal{D}}^{u}(\tilde{\Sigma})^{\prime}, b^{\psi} \in \tilde{\mathcal{D}}^{v}(\tilde{\Sigma})^{\prime}$ with

$$
\psi(f)=a^{\psi}\left(u_{\alpha}(f)\right)+b^{\psi}\left(v_{\alpha}(f)\right), \forall f \in \mathcal{D}(\mathcal{T}) .
$$

Proof. Considered as distributions, the functions $u_{\alpha}$ act as

$$
\begin{gathered}
u_{\alpha}(f)=\left\langle u_{\alpha}, f\right\rangle_{M}=\int_{\mathcal{I}} d t T_{\alpha}(t)\left\langle\zeta_{\alpha}, f(t, .)\right\rangle_{\Sigma_{t}}=\int_{\mathcal{I}} d t T_{\alpha}(t)\left(\zeta_{-\alpha}, \check{\Gamma} f(t, .)\right)_{\Sigma_{t}}= \\
=\int_{\mathcal{I}} d t T_{\alpha}(t) I_{\alpha}(t) \mathcal{F}[\check{\Gamma} f(t, .)](-\alpha), \forall f \in \mathcal{D}(\mathcal{T}) .
\end{gathered}
$$

(Remember that $g_{00}=1$.) The action of $v_{\alpha}$ is similar. By assumption (Eq 2.19) and Remark 2.6 we find

$$
T_{\alpha}(t) I_{\alpha}(t) \mathcal{F}[\check{\Gamma} f(t, .)](-\alpha) \in \tilde{\mathcal{D}}(\tilde{\Sigma}) .
$$

Then by Proposition 2.6 we get $u_{\alpha}(f) \in \tilde{\mathcal{D}}(\tilde{\Sigma})$ (similarly for $v_{\alpha}$ ).

In general, the maps $f \rightarrow u_{\alpha}(f)$ and $f \rightarrow v_{\alpha}(f)$ need not be surjective. Therefore we define

$$
\tilde{\mathcal{D}}^{u}(\tilde{\Sigma})=u_{\alpha}(\mathcal{D}(\mathcal{T}))
$$

By continuity of the map $f \rightarrow u_{\alpha}(f)$ (which is easy to establish), $\tilde{\mathcal{D}}^{u}(\tilde{\Sigma})$ is a closed subspace of $\tilde{\mathcal{D}}(\tilde{\Sigma})$. Similarly we define $\tilde{\mathcal{D}}^{v}(\tilde{\Sigma})$.

Recall the mode expansion for arbitrary $\phi \in \operatorname{Sol}_{0}(\mathcal{T})$,

$$
\phi(t, \vec{x})=\int_{\tilde{\Sigma}} d \mu(\alpha)\left[a_{\alpha}^{\phi} u_{\alpha}(x)+b_{\alpha}^{\phi} v_{\alpha}(x)\right] .
$$

Thus $\operatorname{Sol}_{0}(\mathcal{T})$ can be written as a direct sum of linear subspaces, $\operatorname{Sol}_{0}(\mathcal{T})=$ $\operatorname{Sol}_{0}^{u}(\mathcal{T}) \oplus \operatorname{Sol}_{0}^{v}(\mathcal{T})$, with

$$
\operatorname{Sol}_{0}^{u}(\mathcal{T})=\left\{\phi \in \operatorname{Sol}_{0}(\mathcal{T}): b^{\phi}=0\right\}, \operatorname{Sol}_{0}^{v}(\mathcal{T})=\left\{\phi \in \operatorname{Sol}_{0}(\mathcal{T}): a^{\phi}=0\right\},
$$


and we will write $\phi=\phi^{u}+\phi^{v}$. Regarding as a distribution in $\mathcal{D}(\mathcal{T})^{\prime}, \phi^{u}$ and $\phi^{v}$ act as

$$
\phi^{u}(f)=\int_{\tilde{\Sigma}} d \nu(\alpha) a_{\alpha}^{\phi} u_{\alpha}(f), \phi^{v}(f)=\int_{\tilde{\Sigma}} d \nu(\alpha) b_{\alpha}^{\phi} v_{\alpha}(f) .
$$

The functions $a_{\alpha}^{\phi}, b_{\alpha}^{\phi}$ can be regarded as distributions $a^{\phi} \in \tilde{\mathcal{D}}^{u}(\tilde{\Sigma})^{\prime}, b^{\phi} \in \tilde{\mathcal{D}}^{v}(\tilde{\Sigma})^{\prime}$, and we can write

$$
\phi(f)=\phi^{u}(f)+\phi^{v}(f)=a^{\phi}\left(u_{\alpha}(f)\right)+b^{\phi}\left(v_{\alpha}(f)\right) .
$$

Now let $\varphi \in \operatorname{Sol}(\mathcal{T})$ be a solution, which does not necessarily have $\operatorname{supp}\{\varphi\} \cap \Sigma_{t}$ compact. Its Cauchy data are

$$
\left(i_{t}^{*}(\varphi), i_{t}^{*}\left(\nabla_{t} \varphi\right)\right)=\left(\varphi_{0}, \varphi_{1}\right) \in \mathcal{E}\left(\mathcal{T}_{t}\right) \oplus \mathcal{E}\left(\mathcal{T}_{t}\right) .
$$

Choosing a countable (compactly finite) partition of unity on $\Sigma$ we can write

$$
\left(\varphi_{0}, \varphi_{1}\right)=\sum_{i=1}^{\infty}\left(\phi_{0}^{i}, \phi_{1}^{i}\right),\left(\phi_{0}^{i}, \phi_{1}^{i}\right) \in \mathcal{D}\left(\mathcal{T}_{t}\right) \oplus \mathcal{D}\left(\mathcal{T}_{t}\right),
$$

where the sum involves finite items on any compact region $U \in \Sigma$. Now for each $i$ we have

$$
\phi^{i}=i_{t}^{-1}\left(\phi_{0}^{i}, \phi_{1}^{i}\right) \in \operatorname{Sol}_{0}(\mathcal{T})
$$

thus

$$
\phi^{i}=\phi^{i, u}+\phi^{i, v}=i_{t}^{-1}\left(\phi_{0}^{i, u}, \phi_{1}^{i, u}\right)+i_{t}^{-1}\left(\phi_{0}^{i, v}, \phi_{1}^{i, v}\right), \phi^{i, u} \in \operatorname{Sol}_{0}^{u}(\mathcal{T}), \phi^{i, v} \in \operatorname{Sol}_{0}^{v}(\mathcal{T})
$$

Set

$$
\varphi^{u}=\sum_{i=1}^{\infty} i_{t}^{-1}\left(\phi_{0}^{i, u}, \phi_{1}^{i, u}\right)=\sum_{i=1}^{\infty} \phi^{i, u},
$$

and

$$
\varphi^{v}=\sum_{i=1}^{\infty} i_{t}^{-1}\left(\phi_{0}^{i, v}, \phi_{1}^{i, v}\right)=\sum_{i=1}^{\infty} \phi^{i, v},
$$

where the sums converge in compact topology. (This can be seen as follows. The intersection of the causal cone of any compact region with a Cauchy surface is a compact surface, and therefore only finite summands survive.) But we have

$$
\phi^{i, u}(f)=a^{\phi^{i}}\left(u_{\alpha}(f)\right), \phi^{i, v}(f)=b^{\phi^{i}}\left(v_{\alpha}(f)\right)
$$

for some distributions $a^{\phi^{i}}$ and $b^{\phi^{i}}$. Thus we obtain

$$
\varphi^{u}(f)=\sum_{i=1}^{\infty} \phi^{i, u}(f)=\sum_{i=1}^{\infty} a^{\phi^{i}}\left(u_{\alpha}(f)\right)
$$

and

$$
\varphi^{v}(f)=\sum_{i=1}^{\infty} \phi^{i, v}(f)=\sum_{i=1}^{\infty} b^{\phi^{i}}\left(v_{\alpha}(f)\right) .
$$


This convergence defines distributions

$$
a^{\varphi}=\sum_{i=1}^{\infty} a^{\phi^{i}} \in \tilde{\mathcal{D}}^{u}(\tilde{\Sigma})^{\prime}, b^{\varphi}=\sum_{i=1}^{\infty} b^{\phi^{i}} \in \tilde{\mathcal{D}}^{v}(\tilde{\Sigma})^{\prime}
$$

such that

$$
\varphi^{u}(f)=a^{\varphi}\left(u_{\alpha}(f)\right), \varphi^{v}(f)=b^{\varphi}\left(v_{\alpha}(f)\right), \varphi=\varphi^{u}+\varphi^{v}, \forall f \in \mathcal{D}(\mathcal{T}),
$$

and thus $\operatorname{Sol}(\mathcal{T})=\operatorname{Sol}^{u}(\mathcal{T}) \oplus \operatorname{Sol}^{v}(\mathcal{T})$, where

$$
\operatorname{Sol}^{u}(\mathcal{T})=\left\{\varphi \in \operatorname{Sol}(\mathcal{T}): b^{\varphi}=0\right\}, \operatorname{Sol}^{v}(\mathcal{T})=\left\{\varphi \in \operatorname{Sol}(\mathcal{T}): a^{\varphi}=0\right\} .
$$

Now let $\psi \in \mathcal{D}(\mathcal{T})_{0}^{\prime}$ be a weak solution, and $\left\{\chi_{m}\right\}$ a usual mollifier on $\Sigma_{t}$. Define the mollifications $\nabla_{m} \psi \in \operatorname{Sol}(\mathcal{T})$ by

$$
\bigcirc_{m} \psi=\jmath\left(\chi_{m} \psi_{0}, \chi_{m} \psi_{1}\right)
$$

where $\psi=\jmath\left(\psi_{0}, \psi_{1}\right)$ by Proposition 2.7 . Then it is easy to see that $\varnothing_{m} \psi \rightarrow \psi$ in $\mathcal{D}(\mathcal{T})^{\prime}$. That $\varnothing_{m} \psi \in \operatorname{Sol}(\mathcal{T})$ it follows

$$
\bigcirc_{m} \psi=\left(\bigcirc_{m} \psi\right)^{u}+\left(\bigcirc_{m} \psi\right)^{v},\left(\bigcirc_{m} \psi\right)^{\bullet} \in S o l^{\bullet}(\mathcal{T}) .
$$

The disjointness $\operatorname{Sol}^{u}(\mathcal{T}) \cap \operatorname{Sol}^{v}(\mathcal{T})=0$ implies that $\left(\bigcirc_{m} \psi\right)^{u} \rightarrow \psi^{u}$ and $\left(\bigcirc_{m} \psi\right)^{v} \rightarrow$ $\psi^{v}$ with some distributions $\psi^{u} \in \overline{\operatorname{Sol}^{u}(\mathcal{T})}, \psi^{v} \in \overline{\operatorname{Sol}^{v}(\mathcal{T})}$, such that $\psi=\psi^{u}+\psi^{v}$. We denote

$$
\begin{aligned}
& \psi^{u}(f)=\lim _{m \rightarrow \infty}\left(\bigcirc_{m} \psi\right)^{u}(f)=\lim _{m \rightarrow \infty} a^{\psi_{m}}\left(u_{\alpha}(f)\right) \doteq a^{\psi}\left(u_{\alpha}(f)\right), \\
& \psi^{v}(f)=\lim _{m \rightarrow \infty}\left(\bigcirc_{m} \psi\right)^{v}(f)=\lim _{m \rightarrow \infty} b^{\psi_{m}}\left(v_{\alpha}(f)\right) \doteq b^{\psi}\left(v_{\alpha}(f)\right),
\end{aligned}
$$

for some distributions $a^{\psi}$ and $b^{\psi}$. Finally we arrive at

$$
\psi(f)=a^{\psi}\left(u_{\alpha}(f)\right)+b^{\psi}\left(v_{\alpha}(f)\right), \forall f \in \mathcal{D}(\mathcal{T}) .
$$

The map

$$
\mathcal{D}(\mathcal{T})_{0}^{\prime} \ni \psi \rightarrow\left(a^{\psi}, b^{\psi}\right) \in \tilde{\mathcal{D}}^{u}(\tilde{\Sigma})^{\prime} \oplus \tilde{\mathcal{D}}^{v}(\tilde{\Sigma})^{\prime}
$$

is a bijection by construction.

\subsection{The propagator}

In this section we will find the explicit form of the propagator $E$ in terms of the mode decomposition. Of course, Green's functions can be calculated using the techniques of inverse operators. But our approach will be more concordant to the spirit of this work and will at the same time demonstrate the usefulness of the mode decomposition in general.

To use the mode decomposition for weak solutions we assume that at least the condition (iv) of the conventional Fourier transform holds, and that the assumptions of Proposition 2.8 are satisfied. Choose mode solutions to be such that $T_{\alpha}(0)=$ 
$T_{-\alpha}(0)$ and $\dot{T}_{\alpha}(0)=\dot{T}_{-\alpha}(0)$. Then because $\alpha \rightarrow-\alpha$ preserves both $\lambda_{\alpha}(t)$ and the component $\tilde{\Sigma}^{i}$, we have the same mode equations for $T_{\alpha}$ and $T_{-\alpha}$, hence everywhere $T_{\alpha}(t)=T_{-\alpha}(t)$.

The function

$$
\operatorname{det} W\left[T_{\alpha}, \bar{T}_{\alpha}\right](t)=I_{\alpha}(t)\left[\dot{T}_{\alpha}(t) \bar{T}_{\alpha}(t)-T_{\alpha}(t) \dot{\bar{T}}_{\alpha}(t)\right] \in C^{\infty}(\mathcal{I}, i \cdot \mathbb{R})
$$

is the Wronskian of two independent solutions $T_{\alpha}$ and $\bar{T}_{\alpha}$ and is therefore an imaginary constant. For convenience we appoint once and forever to consider only the modes normalized by

$$
\dot{T}_{\alpha}(t) \bar{T}_{\alpha}(t)-T_{\alpha}(t) \dot{\bar{T}}_{\alpha}(t)=i \cdot I_{\alpha}^{-1}(t) .
$$

It can be seen that this condition is consistent with our previous assumptions for the modes $T_{\alpha}$.

We remark that the Krein space involution $\check{\Gamma}$ commutes with the connection components $\Gamma_{i}$. Indeed, by definition $\check{\Gamma}=P^{+}-P^{-}$, where $P^{ \pm}$are the projections onto the subspaces of positive/negative definiteness of the metric $\langle,\rangle_{g}$. Let $\left\{e_{i}\right\}$ be a pseudo-orthonormal moving frame, i.e., $\left\langle e_{i}, e_{i}\right\rangle_{g}= \pm 1$. The value of each $\left\langle e_{i}, e_{i}\right\rangle_{g}$ is preserved under $\nabla$, and therefore $e_{i}$ remains in the same eigenspace of $\check{\Gamma}$, although in our main frame $e_{i}$ experiences gradient,

$$
\nabla e_{i}=\sum_{j=1}^{4} \sum_{k=1}^{n} \Gamma_{j i}^{k} d x^{j} \otimes e_{k} .
$$

Hence $\check{\Gamma}$ commutes with all $\Gamma_{i}$. We have that

$$
\langle u, v\rangle_{\Sigma_{t}}=(\check{\Gamma} \bar{u}, v)_{\Sigma_{t}}=\int_{\tilde{\Sigma}} d \mu(\alpha) s(\alpha) I_{\alpha}(t) \tilde{u}(-\alpha) \tilde{v}(\alpha), \forall u \in \mathcal{E}\left(\mathcal{T}_{t}\right), v \in \mathcal{D}\left(\mathcal{T}_{t}\right),
$$

where $s(\alpha)$ is the Fourier image of the Krein involution $\check{\Gamma}$, which due to the remark above satisfies $s\left(\tilde{\Sigma}^{i}\right)=\{+1,-1\}$, i.e., is constant on each component $\tilde{\Sigma}^{i}$. We have used the fact that $\overline{\tilde{u}}(\alpha)=\tilde{u}(-\alpha)$ which follows from the condition (iv) of the conventional Fourier transform.

Now the propagator is the unique operator $E: \mathcal{D}(\mathcal{T}) \rightarrow \operatorname{Sol}_{0}(\mathcal{T})$ which satisfies

$$
\begin{array}{r}
v(f)=\langle v(t ; .), \dot{E}[f](t ; .)\rangle_{\Sigma_{t}}-\langle\dot{v}(t ; .), E[f](t ; .)\rangle_{\Sigma_{t}}, \\
\forall v \in \operatorname{Sol}_{0}(\mathcal{T}), f \in \mathcal{D}(\mathcal{T}), t \in \mathcal{I} .
\end{array}
$$

As $v \in S_{0} l_{0}(\mathcal{T})$ we can write

$$
v(x)=\int_{\tilde{\Sigma}} d \mu(\alpha) a^{v}(\alpha) u_{\alpha}(x)+\int_{\tilde{\Sigma}} d \mu(\alpha) b^{v}(\alpha) v_{\alpha}(x),
$$

and

$$
\begin{aligned}
& \widetilde{v(t ; .)}(\alpha)=a^{v}(\alpha) T_{\alpha}(t)+b^{v}(\alpha) \bar{T}_{\alpha}(t), \\
& \widetilde{\dot{v}(t ; .)}(\alpha)=a^{v}(\alpha) \dot{T}_{\alpha}(t)+b^{v}(\alpha) \dot{\bar{T}}_{\alpha}(t) .
\end{aligned}
$$


Similarly for $E[f] \in \operatorname{Sol}_{0}(\mathcal{T})$,

$$
\begin{gathered}
E[f](x)=\int_{\tilde{\Sigma}} d \mu(\alpha) a^{E}[f](\alpha) u_{\alpha}(x)+\int_{\tilde{\Sigma}} d \mu(\alpha) b^{E}[f](\alpha) v_{\alpha}(x), \\
\widetilde{E[f](t ;} ;)(\alpha)=a^{E}[f](\alpha) T_{\alpha}(t)+b^{E}[f](\alpha) \bar{T}_{\alpha}(t),
\end{gathered}
$$

and

$$
\widetilde{E[f](t ;} ;)(\alpha)=a^{E}[f](\alpha) \dot{T}_{\alpha}(t)+b^{E}[f](\alpha) \dot{\bar{T}}_{\alpha}(t),
$$

with some distribution fields $a^{E}[f](\alpha)$ and $b^{E}[f](\alpha)$. Using all this we compute

$$
\begin{gathered}
\langle v(t ; .), \dot{E}[f](t ; .)\rangle_{\Sigma_{t}}-\langle\dot{v}(t ; .), E[f](t ; .)\rangle_{\Sigma_{t}}= \\
=-\int_{\tilde{\Sigma}} d \mu(\alpha) s(\alpha) I_{\alpha}(t)\left[\dot{T}_{\alpha}(t) \bar{T}_{\alpha}(t)-T_{\alpha}(t) \dot{\bar{T}}_{\alpha}(t)\right]\left[a^{v}(-\alpha) b^{E}[f](\alpha)-b^{v}(-\alpha) a^{E}[f](\alpha)\right]=
\end{gathered}
$$

by normalization $(\mathrm{Eq} 2.21)$

$$
=-i \int_{\tilde{\Sigma}} d \mu(\alpha) s(\alpha) I_{\alpha}(t)\left[a^{v}(-\alpha) b^{E}[f](\alpha)-b^{v}(-\alpha) a^{E}[f](\alpha)\right] .
$$

On the other hand, we know that $E D=0$, thus $a^{E}[f](\alpha)$ and $b^{E}[f](\alpha)$ are weak solutions of the field equation and can be mode decomposed as

$$
\begin{gathered}
a^{E}[f](\alpha)=a_{\alpha}^{1}\left(u_{\beta}(f)\right)+a_{\alpha}^{2}\left(v_{\beta}(f)\right), \\
b^{E}[f](\alpha)=b_{\alpha}^{1}\left(u_{\beta}(f)\right)+b_{\alpha}^{2}\left(v_{\beta}(f)\right) .
\end{gathered}
$$

By (Eq2.23) we have

$$
v(f)=\int_{\tilde{\Sigma}} d \mu(\alpha) a^{v}(\alpha) u_{\alpha}(f)+\int_{\tilde{\Sigma}} d \mu(\alpha) b^{v}(\alpha) v_{\alpha}(f) .
$$

Inserting (Eq 2.24), (Eq 2.25) and (Eq2.26) into (Eq 2.22) we obtain

$$
a_{\alpha}^{1}=b_{\alpha}^{2}=0, a_{\alpha}^{2}=-b_{\alpha}^{1}=i \cdot s(\alpha) \delta(\beta-\alpha) .
$$

And our final formula is

$$
E[f](x)=i \int_{\tilde{\Sigma}} d \mu(\alpha) s(\alpha)\left[v_{-\alpha}(f) u_{\alpha}(x)-u_{-\alpha}(f) v_{\alpha}(x)\right],
$$

which is in full accord with the result obtained by [5] for scalar fields on FRW spacetimes. 


\section{Aspects of Harmonic Analysis in Homogeneous Spacetimes}

\subsection{Spatially homogeneous cosmological models}

The main goal of the current work is to refurbish the mathematical framework of quantum field theory on classical cosmological spacetimes, in general, and to advance towards a satisfactory rigorous description of cosmological particle creation in states of low energy for hyperbolic fields, in particular. The latter would be an extension of results obtained in [1] for the Klein-Gordon field on specific FRW models to more general situations. Thus although some results were and will be obtained under abstract general assumptions, our attention is concentrated at the geometrical setup of most common cosmological models. Supported by observations of the universe at large scale, cosmology considers mainly spatially homogeneous, or in addition also isotropic, spacetimes. A condensed account of cosmological arguments and their geometrical implications can be found, for instance, in [18. The essence of these geometrical restrictions is mathematically expressed by imposing the existence of a sufficiently rich system of symmetries (more precisely, a group of spatial isometries) on the spacetime. Extensive treatments of all possible isometry groups and related questions can be found in [18, [19], [20. An introduction to the generalities of harmonic analysis on vector bundles is given in [21]. In this section we will try to deductively introduce our geometrical setup with the help of the information in the above mentioned references.

Foliation by equal time Cauchy hypersurfaces. Recall that we are working with a four dimensional globally hyperbolic Lorentzian manifold $(M, g)$ on which a global smooth time function and an atlas can be chosen following [8] such that $M$ is foliated by three dimensional spacelike equal-time smooth Cauchy hypersurfaces and

$$
d s^{2}=g_{00} d t^{2}-d \sigma^{2},
$$

where $d \sigma^{2}$ is the line element on any of those Cauchy surfaces being Riemannian submanifolds.

The structure group. Any vector bundle $\mathcal{T}$ can be considered as associated to its frame bundle $\mathcal{P}_{\mathcal{T}}$ with structure group $G L(n)$. If we want the fiberwise transformations to respect the fiber metric, then we have to restrict the principal bundle to the orthogonal frame bundle. All fibers $V_{p}$ with their respective non-degenerate pseudo-Riemannian structures $\mathfrak{g}_{p}$ are isomorphic, and their generalized orthogonal groups $O\left(\mathfrak{g}_{p}\right)$ (i.e., groups of invertible endomorphisms of $V_{p}$ preserving $\mathfrak{g}_{p}$ ) are isomorphic to the generalized Lorentz group $O\left( \pm_{\mathfrak{g}}\right)$, where $\pm_{\mathfrak{g}}$ in this context will be understood as the signature of $\mathfrak{g}$. But the same vector bundle $\mathcal{T}$ can be associated also to another principal bundle (which we again denote by $\mathcal{P}_{\mathcal{T}}$ ) with structure group $H$ (say, for field theoretical reasons). Then we have a representation $r$ of $H$ on $V$. If $r$ also respects the metric, then $r(H) \in O\left( \pm_{\mathfrak{g}}\right)$, so $H$ is homomorphic to

$O\left( \pm_{\mathfrak{g}}\right)$. For instance, $H=S O^{+}\left( \pm_{\mathfrak{g}}\right)$ (tensor bundle) or $H=\operatorname{Spin}^{+}\left( \pm_{\mathfrak{g}}\right)$ (spinor bundle). 
Isometries. Let us start with reminding some definitions. An isometry of the spacetime $(M, g)$ is a diffeomorphism $\psi: M \rightarrow M$ such that $\psi^{*} g=g$ holds on $M$, where $\psi^{*}$ is the pullback of $\psi$. If $\psi^{\prime}: M \rightarrow M$ is another isometry, then obviously such is also their superposition $\psi \circ \psi^{\prime}$. With the superposition as product, isometries thus constitute an abstract group, which we will denote $\operatorname{Iso}(M)$. If $\mathcal{T} \rightarrow M$ is a the vector bundle over $M$ as defined previously, then an isometry of the vector bundle $\mathcal{T}$ is a morphism $\Psi: \mathcal{T} \rightarrow \mathcal{T}$ covering an isometry of the base, $\pi \circ \Psi \circ \pi^{-1} \in \operatorname{Iso}(M)$, such that $\Psi^{*} \mathfrak{g}=\mathfrak{g}$ and $\Psi^{*} \nabla=\nabla$ (or more precisely $\Psi^{*} D=D$ when a normal hyperbolic field operator $D$ is specified), where $\Psi^{*}$ denotes pullback maps, $\mathfrak{g}$ is the pseudo-Riemannian fiber metric, and $\nabla$ is the metric connection. Again via superposition, the isometries of the bundle $\mathcal{T}$ comprise an abstract group $\operatorname{Iso}(\mathcal{T})$.

The map $\operatorname{Iso}(\mathcal{T}) \ni \Psi \rightarrow \pi \circ \Psi \circ \pi^{-1} \in \operatorname{Iso}(M)$ gives a homomorphism of $\operatorname{Iso}(\mathcal{T})$ into Iso $(M)$. The image of this homomorphosm is a subgroup of Iso $(M)$ and will be denoted by $\operatorname{Iso}^{\mathcal{T}}(M) \subset \operatorname{Iso}(M)$, and its kernel is a normal subgroup of $\operatorname{Iso}(\mathcal{T})$. This kernel $\mathbf{I s o}(\mathcal{T}) / \mathbf{I s o}^{\mathcal{T}}(M)$ consists of isometries of the bundle $\mathcal{T}$ covering the identity map of $M$. These are precisely the smooth sections in the principle bundle $\mathcal{P}_{\mathcal{T}} \stackrel{\text { loc }}{\longrightarrow} M \times H$ of $\mathcal{T}$, i.e., $\operatorname{Iso}(\mathcal{T}) / \mathbf{I s o}^{\mathcal{T}}(M)=C^{\infty}\left(\mathcal{P}_{\mathcal{T}}\right)$. The group multiplication is given by the pointwise multiplication of sections.

Homogeneous bundle structure. If the sections in the bundle $\mathcal{T}$ are going to represent physical fields, than one should have a concrete picture of how they transform under the diffeomorphisms of the spacetime $M$. In case of the tensor bundle this picture is automatically encoded in the pullback map. An abstract vector bundle does not have such a structure by itself. Thus a physical field theory has to specify a homomorphism $\rho: \operatorname{Diff}(M) \rightarrow C^{\infty}\left(\mathcal{P}_{\mathcal{T}}\right)$. For the tangent bundle $\rho(\psi)=d \psi, \psi \in \operatorname{Diff}(M)$. When considering arbitrary diffeomorphisms, then the structure group should be $G L(n)$ rather than a smaller $H$. But if we restrict $\rho$ to $\rho: \mathbf{I s o}^{\mathcal{T}}(M) \rightarrow C^{\infty}\left(\mathcal{P}_{\mathcal{T}}\right)$, then $H$ can be chosen. For brevity denote $G=\operatorname{Iso}^{\mathcal{T}}(M)$. We have the injection

$$
G \ni g \rightarrow g \times \rho(g) \in \mathbf{I s o}(\mathcal{T})
$$

which gives sense to the left action of $G$ on $\mathcal{T}$ by isometries.

The abstract group of isometries of a pseudo-Riemannian manifold of dimension $m$ is given the compact open topology, in which it becomes a Lie group of dimension at most $n(n+1) / 222$. It can be further shown, that the compact open topology in this case is equivalent to the pointwise convergence topology of isometries. Thus we automatically obtain a Lie group structure on $\operatorname{Iso}(M)$. Then $G \subset \operatorname{Iso}(M)$ is a topological subgroup defined by

$$
G=\left\{\psi \in \mathbf{I} \mathbf{s o}(M):(\psi \times \rho(\psi))^{*} \mathfrak{g}=\mathfrak{g},(\psi \times \rho(\psi))^{*} D=D\right\} .
$$

If $\rho$ is a continuous homomorphism, then all the operations in the equations

$$
(\psi \times \rho(\psi))^{*} \mathfrak{g}=\mathfrak{g},(\psi \times \rho(\psi))^{*} D=D
$$

are continuous, and therefore the subspace $G$ of $\mathbf{I s o}(M)$ defined by this equation is a closed topological subspace. But then by Cartan's theorem $G$ is actually a Lie 
subgroup, as it is a closed topological subgroup of the Lie group Iso $(M)$. Thus we have the structure of a $G$-homogeneous vector bundle $\mathcal{T}$.

Spatially homogeneous bundle. The bundle $\mathcal{T}$ will be called spatially homogeneous if the orbits of $\mathbf{I s o}^{\mathcal{T}}(M)$ are three dimensional smooth spacelike hypersurfaces which foliate $M$. (Maybe it is worth mentioning here that everywhere in this work we consider only connected spacetimes $M$.) By Theorem 8.16 in 20] there exists a parametrization of these orbits by the affine parameter of the family of normal geodesics, such that the metric takes the form

$$
d s^{2}=d t^{2}-d \sigma^{2} .
$$

On the other hand, our original foliation by equal time Cauchy surfaces due to Theorem 1.1 in 8 also yielded such a metric form. We assume that the time function can be chosen such that equal time Cauchy surfaces are the orbits of Iso $^{\mathcal{T}}(M)$ (probably this can be shown to be true in general). We note that due to the transitive action of $G$ on $\Sigma_{t}$ for every $t$, it holds $G \subset$ Iso $^{\mathcal{T}_{t}}\left(\Sigma_{t}\right)$. We did not write $G=\mathbf{I s o}^{\mathcal{T}_{t}}\left(\Sigma_{t}\right)$ because it is possible that for some $t \neq t^{\prime} \in \mathcal{I}$, Iso ${ }^{\mathcal{T}_{t}}\left(\Sigma_{t}\right) \neq$ Iso $^{\mathcal{T}_{t^{\prime}}}\left(\Sigma_{t^{\prime}}\right)$, i.e., for some time instances the time slice may be more symmetric than usual. We will concentrate on $G$, which is the maximal guaranteed amount of symmetry which is present at any time. Thus we see that $\mathcal{T}_{t}$ also has the structure of a $G$-homogeneous vector bundle.

Consider the principle bundle $\mathcal{P}_{\mathcal{T}_{t}}$ of $\mathcal{T}_{t}$, which is a subbundle of $\mathcal{P}_{\mathcal{T}}$. The smooth left action of $G$ on $\mathcal{T}_{t}$ gives a smooth left action of $G$ on $\mathcal{P}_{\mathcal{T}_{t}}$ as well. This action allows one to construct a global smooth section in $\mathcal{P}_{\mathcal{T}_{t}}$, whence it follows that the bundle $\mathcal{T}_{t}$ is trivial. Because $M \sim \Sigma_{t} \times \mathcal{I}$, the whole bundle $\mathcal{T}$ is also trivial. Thus spatially homogeneous vector bundles over $M$ are necessarily trivial.

The requirement that the field operator $D$ is $G$-invariant implies that the function $m^{\star}(x)$ is in fact a function of time only.

Homogeneous space structure. Now let $\operatorname{StabIso}^{\mathcal{T}}(p) \subset G$ be the stabilizer of $G$ at some fixed point $p \in M$. Then $\operatorname{StabIso}^{\mathcal{T}}(p)$ is a closed Lie subgroup by Cartan's theorem. That for all $p \in M$, the groups $\operatorname{StabIso}^{\mathcal{T}}(p)$ are isomorphic, then we denote them all by $\operatorname{StabIso}^{\mathcal{T}}(M)$. In this case the orbits $\Sigma_{t}$ of $G$ are diffeomorphic to the homogeneous space $G / \operatorname{StabIso}^{\mathcal{T}}(M) \doteq \Sigma$. Denote $O=\operatorname{StabIso}^{\mathcal{T}}(M)^{+}$, the identity component. Then $\Gamma=\operatorname{StabIso}^{\mathcal{T}}(M) / O$ is a discrete normal subgroup of $G$. If the homogeneous space $\Sigma$ is itself a Lie subgroup of $G$, then it acts on each $\Sigma_{t}$ simply transitively.

The four dimensional reality. As already mentioned, the isometry group Iso $(M)$ of the $n=4$ dimensional spacetime $M$ is a Lie group of dimension at most $n(n+1) / 2=10$. Thus in principle one can construct all real Lie algebras $\mathcal{G}$ of dimension up to 10, their corresponding connected simply connected Lie groups $G$, then all discrete normal subgroups $\Gamma$ of such $G$ etc., thereby exhausting all possible isometry groups of $M$. This heavy task have been done by Petrov et al [19] and others [20], and all the possibilities are listed in tables. It turned out that only the Minkowski space has isometry group of maximal dimension 10, which is the 
Poincaré group. Among all possibilities we are interested in those whose orbits are $\Sigma_{t}$. Thus the dimension of $G$ is at least three. There are three possibilities of six dimensional such isometry groups, which correspond to FRW spacetimes. A number of possibilities are available with four dimensional groups, which correspond to the LRS spacetimes. And finally there are nine classes of three dimensional real Lie groups $B i(N)$ (called Bianchi groups), which together with their factors $B i(N) / \Gamma$ by discrete subgroups $\Gamma$ represent the isometry groups of the spatially homogeneous spacetimes. It turned out further, that in all these cases besides one (the so called Kantowski-Sachs model) the isometry group is the semidirect product $G=\Sigma \rtimes O$. In this case we will call $\mathcal{T}_{t}$ a semidirect homogeneous vector bundle. In particular, for six dimensional FRW groups, four dimensional LRS groups and three dimensional Bianchi groups $O=S O(3), S O(2)$ and $\{1\}$, respectively. The normal subgroups $\Sigma$ are nothing else than $B i(N) / \Gamma$.

In next sections we will work on the semidirect homogeneous vector bundles. After establishing the necessary mathematical framework, we will obtain results concerning the structure of $G$-invariant homogeneous bi-distributions.

\subsection{On harmonic analysis in semidirect homogeneous vector bundles}

In this section we will collect information on harmonic analysis in $G$-homogeneous vector bundles $\mathcal{T} \rightarrow G / O$ where $G=\Sigma \rtimes O$ which will be useful later in the work. This does not pretend to be self-contained or systematic; quite the contrary, we will introduce mainly what we were not able to find in the literature. Otherwise references will be provided.

Semidirect homogeneous vector bundles. Let $G=\Sigma \rtimes O$, where $O$ is a compact connected type I Lie subgroup, and $\Sigma$ a connected normal type I Lie subgroup. Moreover, we demand that the modular function of $\Sigma$ has a non-trivial kernel, so that the representation theories of both $\Sigma$ and $G$ are well under control by Theorem 7.50 of 23$]$. We note that this is the case for all Bianchi groups which are in fact the only candidates for $\Sigma$ in our context. Let $\Sigma=G / O$ have a Riemannian structure $h$ which is invariant under the left action of $G$. Let further $\mathcal{T} \rightarrow \Sigma$ be an $n$-dimensional (real or complex) vector bundle with standard fiber $V$ and a pseudoRiemannian fiber metric $\mathfrak{g}$. Let there be a smooth left action of $G$ on $\mathcal{T}$ covering the left multiplication of $G$ on the base, such that the fiber metric is invariant under that action. Then we will call $\mathcal{T}$ a semidirect $G$-homogeneous vector bundle. If we choose an orthonormal frame $\left\{X_{i}\right\}$ of $T_{1}^{*} \Sigma$ (or $\left\{Y_{i}\right\}$ of $\left.\mathcal{T}\right|_{1}$ ), and drag it throughout $\Sigma$ using the transitive left action of $G$, we will obtain $G$-invariant global smooth frame $\left\{X_{i}\right\}$ in $T^{*} \Sigma$ (similarly, $\left\{Y_{i}\right\}$ in $\mathcal{T}$ ). Thus both $T^{*} \Sigma$ and $\mathcal{T}$ are trivial bundles. Associated to the Riemannian structure $h$ there is a Laplace operator $\Delta$ acting on sections $f \in C^{\infty}(\mathcal{T})$.

The regular and quasi-regular representations for the line bundle. Suppose $\mathcal{T}$ from above is a line bundle, $n=1$. The left regular representation $L_{g}$ 
of $G$ on $C(G)$ acts as

$$
L_{g} f(x)=f\left(g^{-1} x\right), \forall g, x \in G
$$

Because the Riemannian structure is $G$-invariant, the metric measure $d x$ is a left Haar measure on $G$, and hence $L_{g}$ is a unitary representation on $L^{2}(G)$.

Now any point $x \in G$ can be uniquely written as $x=x_{\Sigma} x_{O}$, where $x_{\Sigma} \in \Sigma$ and $x_{O} \in O$. Let $d x_{\Sigma}$ be the metric driven left $G$-invariant measure on $\Sigma$, and $d x_{O}$ the Lebesgue measure on $O$ normalized to $|O|=1$. Then $d x=d x_{\Sigma} d x_{O}$ gives a left Haar measure on $G$. Functions $f$ on $\Sigma$ are identified with their right $O$ invariant extensions to $G$, i.e., $f(x o)=f(x)=f(x O)$, for any $x \in G, o \in O$. Thus $C(\Sigma) \in C(G)$ (similarly $L^{2}(\Sigma) \in L^{2}(G)$, etc.) and we may consider the restriction $U_{g}$ of the left regular representation $L_{g}$ on $C(G)$ to $C(\Sigma)$. Its action will be given by

$$
U_{g} f\left(x_{\Sigma} O\right)=f\left(g^{-1} x_{\Sigma} O\right), \forall x_{\Sigma} \in \Sigma, g \in G .
$$

The representation $U_{g}$ of $G$ is the left quasi-regular representation, and it is nothing else but the induced representation $\operatorname{Ind}_{O}^{G} 1$. Note that for $O=\{1\}$ we simply have $G=\Sigma$ and $L_{g}=U_{g}$.

Neither $L_{g}$ nor $U_{g}$ need to be irreducible. The central decomposition of $L_{g}$ is

$$
L_{g}=\int_{\hat{G}}^{\oplus} d \nu(\pi) L_{g}(\pi),
$$

where $\nu(\pi)$ is the Plancherel measure and $L_{g}(\pi)=\pi \otimes 1$ is the primary representation composed of $\operatorname{mult}\left(\pi, L_{g}\right)=\operatorname{dim} \bar{\pi} \in[1, \infty]$ copies of $\pi$ [23. The central decomposition of $U_{g}$ will be

$$
U_{g}=\int_{\hat{G}_{\Sigma}}^{\oplus} d \mu(\pi) U_{g}(\pi),
$$

where $\hat{G}_{\Sigma} \subset \hat{G}, d \mu$ is the spectral measure of $U_{g}$ and for $\mu$-almost all $\pi, U_{g}(\pi)$ is a multiple of $\pi$ (multiplicities $\operatorname{mult}\left(\pi, U_{g}\right)$ and the measure $d \mu(\pi)$ need to be determined). The corresponding Hilbert space decompositions are

$$
L^{2}(G)=\int_{\hat{G}}^{\oplus} d \nu(\pi) \mathcal{H}_{\pi} \otimes \mathcal{H}_{\bar{\pi}}
$$

and

$$
L^{2}(\Sigma)=\int_{\hat{G}_{\Sigma}}^{\oplus} d \mu(\pi) \mathcal{H}(\pi),
$$

where $\mathcal{H}(\pi)=\mathcal{H}_{\pi} \otimes \mathbb{C}^{\text {mult }\left(\pi, U_{g}\right)} \subset \mathcal{H}_{\pi} \otimes \mathcal{H}_{\bar{\pi}}$. Here $\mathbb{C}^{\text {mult }\left(\pi, U_{g}\right)}$ symbolizes some Hilbert space of dimension $\operatorname{mult}\left(\pi, U_{g}\right)$ which is finite or infinite.

In the following we will deal with $U_{g}$ keeping in mind that in case $G=\Sigma$ everything reduces to $L_{g}$. 
The operator $\Pi_{\pi}$. Consider for any $\pi \in \hat{G}$ the bounded operator

$$
\Pi_{\pi}=\int_{O} \operatorname{do\pi }(o) \text {. }
$$

Then $\Pi_{\pi}$ is self adjoint,

$$
\Pi_{\pi}^{*}=\int_{O} \operatorname{do\pi }(o)^{*}=\int_{O} \operatorname{do} \pi\left(o^{-1}\right)=\Pi_{\pi} .
$$

Moreover, because $O$ is unimodular, we have

$$
\pi(o) \Pi_{\pi}=\pi(o) \int_{O} d o^{\prime} \pi\left(o^{\prime}\right)=\int_{O} d\left(o o^{\prime}\right) \pi\left(o o^{\prime}\right)=\Pi_{\pi}=\Pi_{\pi} \pi(o), \forall o \in O,
$$

and hence $\Pi_{\pi}$ is a projection,

$$
\Pi_{\pi}^{2}=\int_{O} d o \pi(o) \Pi_{\pi}=\int_{O} d o \Pi_{\pi}=\Pi_{\pi} .
$$

$\Pi_{\pi}$ is a projection onto an invariant subspace of $\left.\pi\right|_{O}$. Recall the operator $D_{\pi}$ of 23$]$ which satisfied $D_{\pi} \pi(x)=\Delta^{\frac{1}{2}}(x) \pi(x) D_{\pi}$, for all $x \in G$. In particular, we find that $D_{\pi} \pi(o)=\pi(o) D_{\pi}$ for all $o \in O$, and consequently, $D_{\pi} \Pi_{\pi}=\Pi_{\pi} D_{\pi}$.

The Fourier transform in $G / O$. The Fourier transform in $\Sigma=G / O$ associated to $U_{g}$ is naturally the restriction of that on $G$ associated to $L_{g}$; for $\mu$-almost all $\pi \in \hat{G}_{\Sigma}$

$$
\hat{f}(\pi)=\pi(f) D_{\pi} \in \mathcal{H}(\pi) .
$$

For any $f \in C_{0}(\Sigma)$ and $\mu$-almost all $\pi \in \hat{G}_{\Sigma}$ we have

$$
\pi(f)=\int_{\Sigma} d x_{\Sigma} \int_{O} d x_{O} f\left(x_{\Sigma} O\right) \pi\left(x_{\Sigma}\right) \pi\left(x_{O}\right)=\int_{\Sigma} d x_{\Sigma} f\left(x_{\Sigma} O\right) \pi\left(x_{\Sigma}\right) \Pi_{\pi} .
$$

As usual we have $\pi\left(U_{g} f\right)=\pi\left(L_{g} f\right)=\pi(g) \pi(f)$ for $g \in G, f \in C_{0}(\Sigma)$. The convolution $f * h$ has the property that if $f \in C_{0}(G)$ and $h \in C_{0}(\Sigma)$ then $f * h \in$ $C_{0}(\Sigma)$. Moreover, it satisfies $\pi(f * h)=\pi(f) \pi(h)$.

The case of arbitrary $\mathcal{T}$. Let now $\operatorname{dim} V=n \geq 1$. The left quasi-regular representation of $G$ on $C^{\infty}(\mathcal{T})$ acts as

$$
U_{g}^{\mathcal{T}} f(x)=g^{-1} f\left(g^{-1} x\right), \forall f \in C^{\infty}(\mathcal{T}) .
$$

Recall the $G$-invariant orthonormal frame $\left\{Y_{i}\right\}_{i=1}^{n}$ in $\mathcal{T}$ and write any $f \in C^{\infty}(\mathcal{T})$ as $f=\sum f^{i} Y_{i}$. Using that $U_{g}^{\mathcal{T}} Y_{i}=Y_{i}$ we find

$$
U_{g}^{\mathcal{T}} f(x)=\sum_{i=1}^{n} U_{g} f^{i} \times Y_{i}
$$

where $U_{g}$ is the left quasi-regular representation of $G$ on $C^{\infty}(\Sigma)$. Thus $U_{g}^{\mathcal{T}}=$ $\oplus_{n} U_{g}$, and the harmonic analysis of $U_{g}^{\mathcal{T}}$ is the same as that of $U_{g}$ except that each primary subrepresentation of $U_{g}^{\mathcal{T}}$ is the $n$-fold copy of the corresponding primary 
subrepresentation of $U_{g}$. Making the identification $C_{0}^{\infty}(\mathcal{T}) \ni f \rightarrow\left\{f^{i}\right\} \in \oplus_{n} C_{0}^{\infty}(\Sigma)$ we find the Fourier transform of $f \in C_{0}^{\infty}(\mathcal{T})$ to be

$$
\hat{f}(\pi)=\oplus_{i=1}^{n} \hat{f}^{i}(\pi),
$$

or to say in words, a matrix with $n$ times more columns than that of a scalar function. The inverse Fourier transform will be

$$
f(x)=\sum_{i=1}^{n} \int_{\hat{G}_{\Sigma}} d \mu(\pi) \operatorname{Tr}\left[D_{\pi} \Pi_{\pi} \pi^{*}(x) \hat{f}^{i}(\pi)\right] \times Y_{i}(x) .
$$

\section{On the Fourier transform of distributions}

Here we will collect miscellaneous facts about distributions and their Fourier transform, which we did not meet in the literature. We continue working with the semidirect homogeneous vector bundle $\mathcal{T}$ with notations established earlier.

Let $\hat{\mathcal{D}}^{\mathcal{T}}\left(\hat{G}_{\Sigma}\right)$ be the image of $D(\mathcal{T})=C_{0}^{\infty}(\mathcal{T})$ under the harmonic analytical Fourier transform $f\left(x_{\Sigma}\right) \rightarrow \hat{f}(\pi)$. As we have already seen, $\hat{f}(\pi)=\oplus_{n} \hat{f}^{i}(\pi)$, hence $\hat{\mathcal{D}}^{\mathcal{T}}\left(\hat{G}_{\Sigma}\right)=\oplus_{n} \hat{\mathcal{D}}(\Sigma)$, where $\hat{\mathcal{D}}(\Sigma)$ is the image under the Fourier transform of $C_{0}^{\infty}(\Sigma) . \hat{\mathcal{D}}^{\mathcal{T}}\left(\hat{G}_{\Sigma}\right)$ inherits the topology of $D(\mathcal{T})$ via the Fourier transform, and one can consider the Fourier transform of distributions $D(\mathcal{T})^{\prime} \ni u \rightarrow \hat{u} \in \hat{\mathcal{D}}^{\mathcal{T}}\left(\hat{G}_{\Sigma}\right)^{\prime}$ given by $\hat{u}(\hat{f})=u(f)$.

The Fourier transform has the remarkable property that it interchanges the local and global behaviors. Namely, the local irregularities of a function $f$ are reflected in the decay properties of $\hat{f}(\pi)$ at large $\pi$, and conversely, the behavior at infinity of $f$ determines the local regularity of $\hat{f}(\pi)$. The precise description of these phenomena requires a thorough functional analytical investigation, which we, unfortunately, have no possibility to perform here.

It is widely known that any distribution restricted to a compact region is of finite order. In 24] the general structure of distributions of finite order has been found for $D\left(\mathbb{R}^{n}\right)$. Following a similar pattern we present here a partial generalization of that result. By Proposition Appendix A.1 let us choose the topology $\left(X_{i}, 2, l^{2}\right)$ for convenience.

Proposition 4.1. Let $\mathcal{T}_{K}$ be an n-dimensional (complex) pseudo-Riemannian vector bundle over a connected parallelizable (pseudo-)Riemannian manifold $K$, and let $\nabla$ be a fiber metric connection. Every $u \in D\left(\mathcal{T}_{K}\right)^{\prime}$ of finite order has a representation

$$
u(f)=\sum_{q \leq k}\left(F_{\alpha, q}, P_{\alpha, q}\left(X_{i}\right) f\right)_{2}, \forall f \in D(\mathcal{T}),
$$

where $F_{\alpha, q} \in L^{2}\left(\mathcal{T}_{K}\right)$ and the smallest possible such $k$ is the order of $u$.

Proof. By our choice

$$
\|f\|_{k}=\sqrt{\sum_{q \leq k}\left\|P_{\alpha, q}\left(X_{i}\right) f\right\|_{2}^{2}}
$$


Let $k$ be the order of $u$, i.e., $u$ is continuous in $\|\cdot\|_{k}$-norm. Define the following linear injective map

$$
\mathcal{V}: D\left(\mathcal{T}_{K}\right) \rightarrow \Phi=\bigoplus_{q \leq k} L^{2}\left(\mathcal{T}_{K}\right)
$$

by

$$
\mathcal{V}(f)=\bigoplus_{q \leq k} P_{\alpha, q}\left(X_{i}\right) f
$$

Then obviously $\|\mathcal{V}(f)\|_{\Phi}=\|f\|_{k}$. If we denote by $\Psi=\mathcal{V}\left(D\left(\mathcal{T}_{K}\right)\right) \subset \Phi$, then $u \circ \mathcal{V}^{-1}$ is a continuous functional on $\Psi$ with the norm $\|\cdot\|_{\Phi}$, and thus by HahnBanach theorem can be extended to a continuous functional $F \in \Phi^{\prime}$. But $\Phi$ is a Hilbert space, thus $\Phi^{\prime}=\Phi$ and $F \in \Phi$, and for any $\phi \in \Phi$,

$$
F(\phi)=\sum_{q \leq k}\left(F_{\alpha, q}, \phi_{\alpha, q}\right)_{2}, F_{\alpha, q} \in L^{2}(\mathcal{T}) .
$$

This yields our desired formula

$$
u(f)=\sum_{q \leq k}\left(F_{\alpha, q}, P_{\alpha, q}\left(X_{i}\right) f\right)_{2} .
$$

If such a formula held for a smaller $k$, then obviously the order of $u$ would be smaller.

Several variations of this proposition may be established by choosing different norms. Note that the order of a distribution, if finite, depends on the choice of the family of norms defining the topology.

Remark 4.1. As already mentioned, any distribution is locally of finite order, hence the proposition applies to the restriction $u_{K} \in C_{0}^{\infty}\left(\left.\mathcal{T}\right|_{K}\right)^{\prime}$ of any $u \in D(\mathcal{T})^{\prime}$ to arbitrary compact connected region $K \subset \Sigma$.

We come back to our homogeneous bundle $\mathcal{T}$ and proceed to the Fourier description of distributions $u \in D(\mathcal{T})^{\prime}$ of finite order, which again can be applied for restrictions to compact regions.

Proposition 4.2. Any distribution $u \in D(\mathcal{T})^{\prime}$ of finite order is given by

$$
u(f)=\int_{\hat{G}_{\Sigma}} d \mu(\pi) \operatorname{Tr}\left[\hat{u}(\pi)^{*} \hat{f}(\pi)\right],
$$

where $\hat{u}(\pi): \mathbb{C}^{\text {mult }\left(\pi, U_{g}\right) * n} \rightarrow \mathcal{H}_{\pi}$ is a $\mu$-locally integrable field of Hilbert-Schmidt operators. (Note that the trace operator includes also the summation by fiber indices $i=1, . ., n$, which now enumerate blocks of columns.)

Proof. Let $k$ be the order of $u$. Choose $\left\{X_{i}\right\}$ to be the generators of left translations on $C^{\infty}(\mathcal{T})$ and let by Proposition 4.1 write $u$ as

$$
u(f)=\sum_{q \leq k}\left(F_{\alpha, q}, P_{\alpha, q}\left(X_{i}\right) f\right)_{2} .
$$


Consider the Fourier transform

$$
\widehat{X_{i} f}(\pi)=\int_{\Sigma} d x_{\Sigma}\left(\lim _{t \rightarrow 0} \frac{\left(U_{\exp \left(-t \xi_{i}\right)}-1\right) f\left(x_{\Sigma}\right)}{t}\right) \pi\left(x_{\Sigma}\right) \Pi_{\pi} D_{\pi}
$$

where $\xi_{i}$ is the corresponding element of the Lie algebra of $\Sigma$. The integral runs over a compact region, and is therefore uniformly absolutely convergent with the Hilbert-Schmidt norm, thus we can interchange the limit with the integral,

$$
\begin{gathered}
\widehat{X_{i} f}(\pi)=\lim _{t \rightarrow 0} \frac{1}{t} \int_{\Sigma} d x_{\Sigma}\left(U_{\exp \left(-t \xi_{i}\right)}-1\right) f\left(x_{\Sigma}\right) \pi\left(x_{\Sigma}\right) \Pi_{\pi} D_{\pi}= \\
=\lim _{t \rightarrow 0} \frac{\pi\left(\exp \left(-t \xi_{i}\right)\right)-1}{t} \hat{f}(\pi) .
\end{gathered}
$$

On the right hand we see nothing else but the generator of the derived representation of $\pi$,

$$
\lim _{t \rightarrow 0} \frac{\pi\left(\exp \left(-t \xi_{i}\right)\right)-1}{t}=-\partial_{i} \pi
$$

whence we find

$$
\widehat{X_{i} f}(\pi)=-\partial_{i} \pi \hat{f}(\pi)
$$

As a result we have

$$
\left.\widehat{P_{\alpha, q}\left(X_{i}\right.}\right) f(\pi)=P_{\alpha, q}\left(-\partial_{i} \pi\right) \hat{f}(\pi)
$$

and thereby

$$
u(f)=\sum_{q \leq k} \int_{\hat{G}_{\Sigma}} d \mu(\pi) \operatorname{Tr}\left[\hat{F}_{\alpha, q}(\pi)^{*} P_{\alpha, q}\left(-\partial_{i} \pi\right) \hat{f}(\pi)\right]=\int_{\hat{G}_{\Sigma}} d \mu(\pi) \operatorname{Tr}\left[\hat{u}(\pi)^{*} \hat{f}(\pi)\right],
$$

where

$$
\hat{u}(\pi)=\sum_{q \leq k}\left[P_{\alpha, q}\left(-\partial_{i} \pi\right)\right]^{*} \hat{F}_{\alpha, q}(\pi) .
$$

This completes the proof.

Such a result should not be surprising. If the measurable functions $F_{\alpha, q}$ were $q$ times differentiable within the space of locally integrable functions, then we could hypothetically use integration by parts to make all the terms in the formula of Proposition 4.1 of order 0 , which would correspond to a regular distribution. The failure of the derivatives of $F_{\alpha, q}$ to remain locally integrable is reflected in the fact, that multiplication of $\widehat{F}_{\alpha, q}(\pi)$ by $\partial_{i} \pi^{*}$ makes it not square integrable any more, but possibly only locally integrable. This reflects the local-to-global interchange made by the Fourier transform: higher frequencies feel local irregularities.

The image $\hat{\mathcal{D}}^{\mathcal{T}}\left(\hat{G}_{\Sigma}\right)$ of compactly supported smooth sections under the Fourier transform is of considerable interest. In harmonic analysis it is described by various Paley-Wiener type theorems. Although there are refined Paley-Wiener theorems for adapted Fourier transforms for certain classes of semisimple or solvable groups, 
there seems to be no such theory for the general abstract setup. Next we present a partial answer to the problem, namely, a criterion for smoothness for sufficiently decaying functions, which gives hints about how the general solution might look like.

Proposition 4.3. For a function $f \in L^{2}(\mathcal{T})$ the following two conditions are equivalent:

(i) for any polynomial $P\left(X_{i}\right)$ of generators $\left\{X_{i}\right\}$ with constant coefficients, $P\left(X_{i}\right) f \in L^{2}(\mathcal{T})$

(ii) $\hat{f}(\pi)$ decays at infinity of $\hat{G}_{\Sigma}$ faster than the inverse of any polynomial in the generators $\partial_{i} \pi^{*}$

Proof. As we have seen in the proof of the previous proposition,

$$
\widehat{P\left(X_{i}\right)} f=P\left(-\partial_{i} \pi\right)^{*} \hat{f}(\pi),
$$

and the requirement that $\widehat{P\left(X_{i}\right)} f \in L^{2}\left(\hat{G}_{\Sigma}\right)$ for any $P\left(X_{i}\right)$ is equivalent to the assertion (ii) of the proposition.

We can go a step further and establish a weaker necessary condition for a distribution to be given by a smooth integral kernel. For this purpose we want to remind a few definitions on a more abstract level.

Let $\mathcal{D}(S)$ be a test function space. We have $\mathcal{D}(S) \subset L^{\infty}(S)$ and therefore $L^{\infty}(S)^{\prime} \subset \mathcal{D}(S)^{\prime}$. Let $\left\{\eta_{i}\right\}$ be a finite system of linear maps $\eta_{i}: S \rightarrow S$. A distribution $u \in \mathcal{D}(S)^{\prime}$ is of rapid decay in $\left\{\eta_{i}\right\}$ if for any polynomial $P\left(\eta_{i}\right)$ of variables $\left\{\eta_{i}\right\}$ it holds $u\left(P\left(\eta_{i}\right)\right.$.) $\in L^{\infty}(S)^{\prime}$. We will symbolically write this as $u=\mathfrak{o}\left(\left\{\eta_{i}\right\}^{-\infty}\right)$. If $u$ is given by a locally integrable kernel, and $\left\{\eta_{i}\right\}$ are coordinate operators, then this definition obviously reduces to the usual criterion for functions of rapid decay.

Proposition 4.4. For a distribution $u \in \mathcal{D}(\mathcal{T})^{\prime}$ from $\hat{\mathcal{D}}^{\mathcal{T}}\left(\hat{G}_{\Sigma}\right)^{\prime} \ni \hat{u}=\mathfrak{o}\left(\left\{\partial_{i} \pi\right\}^{-\infty}\right)$ it follows that $u$ has a smooth integral kernel.

Proof. That $u$ is smooth means that all derivatives of all fiber components $u^{j}$ are continuous. In other words, for any polynomial $P$ in the generators $\left\{X_{i}\right\}$, the distributions $P\left(X_{i}\right) u^{j}$ can be evaluated pointwise. A precise statement can be given as follows. $u$ is smooth if and only if for any polynomial $P\left(X_{i}\right)$, point $m \in \Sigma$ and sequence of test functions $f_{q} \rightarrow \delta(x-m)$ in $C_{0}^{\infty}(\Sigma)^{\prime}$, the following limit exists for all $j=1, \ldots, n$ and is finite, $\lim _{q \rightarrow \infty} u^{j}\left(P\left(-X_{i}\right) f_{q}\right)$. The Fourier transform of the distribution $\delta_{m}=\delta(x-m)$ can be easily read from the Fourier inversion formula,

$$
\hat{\delta}_{m}(\hat{f})=\int_{\hat{G}_{\Sigma}} d \mu(\pi) \operatorname{Tr}\left[D_{\pi} \Pi_{\pi} \pi^{*}(m) \hat{f}(\pi)\right] .
$$

That means $f_{q} \rightarrow \delta(x-m)$ is equivalent to $\hat{f}_{q} \rightarrow \pi(m) \Pi_{\pi} D_{\pi}$ in the weak sense. Hence

$$
\widehat{P\left(-X_{i}\right)} f_{q} \rightarrow P\left(\partial_{i} \pi\right) \pi(m) \Pi_{\pi} D_{\pi}
$$


in the weak topology. It follows

$$
\lim _{q \rightarrow \infty} u^{j}\left(P\left(-X_{i}\right) f_{q}\right)=\hat{u}^{j}\left(P\left(\partial_{i} \pi\right) \pi(m) \Pi_{\pi} D_{\pi}\right)
$$

whenever one of the sides converges.

Now suppose $\hat{u}=\mathfrak{o}\left(\left\{\partial_{i} \pi\right\}^{-\infty}\right)$. Then for any $\hat{f} \in L^{\infty}\left(\hat{G}_{\Sigma}\right)$ (i.e., $\|\hat{f}(\pi)\| \in$ $L^{\infty}\left(\hat{G}_{\Sigma}\right)$ in the usual sense) we have

$$
\hat{u}^{j}\left(P\left(\partial_{i} \pi\right) \hat{f}^{j}(\pi)\right)<\infty, j=1, \ldots, n .
$$

In particular, $\pi(m) \Pi_{\pi} D_{\pi} \in L^{\infty}\left(\hat{G}_{\Sigma}\right)$, whence (Eq4.1) follows.

We are incline to think that this necessary condition is not far from the desirable equivalent condition. This is, however, an open problem in harmonic analysis, and we only hope to be able to give a satisfactory answer in the future at least in the context we are interested in.

\section{The adapted Fourier transform}

We start by noting that because the function $m^{\star}(t)$ is a function of time only, the eigenfunctions of $D_{\Sigma_{t}}$ are nothing else but the eigenfunctions of the Laplace operator $\Delta_{t}$. In the first chapter we introduced the eigenfunction decomposition associated to any self adjoint operator as the Laplace operator $\Delta$,

$$
f \rightarrow \tilde{f}(\alpha)=\zeta_{\alpha}(f)
$$

where $\zeta_{\alpha}$-s are the generalized eigenfunctions of $\Delta$. Putting additional structure related with particular geometries one arrives at various Fourier transforms, which are very practical in many respects. On the other hand, the abstract harmonic analytical Fourier transform is a powerful tool for analyzing general problems and properties, but its machinery is functional analytically complicated for use. These two theories are, however, related, although the exact relations have not been sufficiently explored in the literature so far except for compact groups. In the compact case the eigenfunctions of $\Delta$ are the matrix elements of the irreducible representations for some choice of the basis, and the two techniques can be unified. Each choice of the basis results in a Fourier transform which is adapted to it, hence such transforms are sometimes called adapted Fourier transforms. In the non-compact case functional analytical complications arise, though intuitively the situation remains similar. In this section we will try to construct adapted Fourier transforms at least on our semidirect homogeneous bundle $\mathcal{T}$.

The Laplace operator $\Delta$ is invariant under $G$ and hence commutes with $U_{g}^{\mathcal{T}}$. This means on each primary component it acts as a multiplication from the right by a possibly unbounded self-adjoint operator $\hat{\Delta}(\pi)$,

$$
\widehat{\Delta f}(\pi)=\hat{f}(\pi) \hat{\Delta}(\pi) .
$$


For any $f \in L^{2}(\mathcal{T})$ we have that $\Delta f$ is a distribution of order at most two. By Proposition 4.2 it means that the multiplication of any Hilbert-Schmidt operator $\hat{f}(\pi)$ by $\hat{\Delta}(\pi)$ from the right leaves it again Hilbert-Schmidt. Let $\sigma(\pi) \subset \mathbb{R}$ be the spectrum of the self-adjoint operator $\hat{\Delta}(\pi)$ as acting from the right (this spectrum is non-positive, because $\Delta$ is an elliptic operator). For each $\lambda \in \sigma(\pi)$ let $\hat{\xi}_{\pi, \lambda, r, s}$ be the generalized eigenfunctions of $\hat{\Delta}(\pi)$, i.e., distributions satisfying $\hat{\xi}_{\pi, \lambda, r, s} \hat{\Delta}(\pi)=$ $\lambda \hat{\xi}_{\pi, \lambda, r, s}$ which are linearly independent and complete in $\mathcal{H}(\pi)$ for $r \in R_{\pi} \subset \mathbb{R}$ and $s \in S_{\pi, \lambda}^{n} \subset \mathbb{R}$ (they can be constructed from delta functions using the spectral theorem). Now consider the following distributions in the Fourier space,

$$
\hat{\zeta}_{\pi, \lambda, r, s}\left(\pi^{\prime}\right)=\delta\left(\pi-\pi^{\prime}\right) \hat{\xi}_{\pi, \lambda, r, s} .
$$

Their preimages are distributions $\zeta_{\pi, \lambda, r, s} \in D(\mathcal{T})^{\prime}$ which are generalized eigenfunctions of $\Delta$, and by elliptic regularity theorem, are smooth sections in $\mathcal{T}$. Thus we have found, that the adapted Fourier transform $\tilde{f}(\pi, \lambda, r, s)$ is nothing else but the coefficients of $\hat{f}(\pi)$ as expended in the system $\hat{\xi}_{\pi, \lambda, r, s}$. It is worth noting that $r$ parameterizes $\mathcal{H}_{\pi}$, and $\lambda, s$ parameterize $\mathbb{C}^{\text {mult }\left(\pi, U_{g}\right)} * n$. Actually, $S_{\pi, \lambda}^{n}$ consists of $n$ copies of some set $S_{\pi, \lambda}$.

The choice of the system $\hat{\xi}_{\pi, \lambda, r, s}$ is rather arbitrary and leaves room for adaptations. The first adaptation we wish to make is the following. For any $\zeta_{\pi, \lambda, r, s}$ we want $\bar{\zeta}_{\pi, \lambda, r, s}=\zeta_{\pi^{\prime}, \lambda^{\prime}, r^{\prime}, s^{\prime}}$ for some other parameters. Obviously $\lambda=\lambda^{\prime}$, and it is easy to see from the Fourier inversion formula, that this amounts to requiring that $\bar{\xi}_{\pi, \lambda, r, s}$ enters the system $\xi_{\bar{\pi}, \lambda, r^{\prime}, s^{\prime}}$ for the representation $\bar{\pi}$ with some other parameters $r^{\prime}$, $s^{\prime}$. The representation $\bar{\pi}$ may lie in the same equivalence class $[\pi]$ or not.

Lie groups are analytic manifolds, and all the group and algebra structure is given by analytic functions in any analytic atlas. In particular, the eigenfunction problem $\Delta \zeta_{\pi, \lambda, r, s}=\lambda \zeta_{\pi, \lambda, r, s}$ is an analytic elliptic equation, and the solutions $\zeta_{\pi, \lambda, r, s}(x)$ are therefore analytic functions in $x$. If $\Sigma$ is compact, then $\hat{G}_{\Sigma}$ is discrete, and each $\sigma(\pi)$ is also discrete. Representations are finite dimensional, hence $r$ and $s$ run over finite sets. The set $\tilde{\Sigma}=\{\alpha=(\pi, \lambda, r, s)\}$ can be considered a discrete manifold symbolically divided into $n$ components as corresponding to each copy of $S_{\pi, \lambda}$. The space $\hat{\mathcal{D}}^{\mathcal{T}}\left(\hat{G}_{\Sigma}\right)$ corresponds now to the space $\tilde{D}(\tilde{\Sigma})$ of functions on $\tilde{\Sigma}$, which are of rapid decay in $\lambda$, and also decay sufficiently fast in $\pi$ by Proposition 4.3 .

If $\Sigma$ is non-compact, suppose there exists a subset $\tilde{K} \subset \hat{G}_{\Sigma}$ such that $\mu\left(\hat{G}_{\Sigma} \backslash \tilde{K}\right)=0$ and $\tilde{K}$ can be cast into an analytic manifold. Then we can restrict our Fourier transform from $\hat{G}_{\Sigma}$ to $\tilde{K}$ without violation of the Plancherel equality. Suppose further that the set $\tilde{\Sigma}=\{\alpha=(\pi, \lambda, r, s)\}$ can be made an analytic manifold consisting of $n$ disjoint components as in the compact case. Each component itself may have several connected components if $1<\operatorname{mult}\left(\pi, U_{g}\right)<\infty$, in which case $s$ will run over a discrete set. Then we can choose $\zeta_{\pi, \lambda, r, s}$ to be analytic in all its parameters (if $s$ is discrete, analyticity in $s$ is void), so that $\hat{\mathcal{D}}^{\mathcal{T}}\left(\hat{G}_{\Sigma}\right)$ will correspond to the space $\tilde{D}(\tilde{\Sigma})$ of some analytic functions on $\tilde{\Sigma}$ which have at least above mentioned decay properties in $\lambda$ and $\pi$, but also are $L^{2}$ in $r$, and in $s$ if the 
latter is continuous.

Finally let us define a symbolic involution $\alpha \rightarrow-\alpha$ on $\tilde{\Sigma}$ satisfying $\zeta_{-\alpha}=$ $\bar{\zeta}_{\alpha}$. Clearly this involution will preserve $\lambda$. Now if the necessary assumptions are satisfied, we arrive at a conventional Fourier transform. In the next publication we will see that in the majority of situations in cosmology these assumptions are valid, and that will enable us to exploit the machinery of mode decomposition to our cosmological models.

\subsection{Invariant bi-distributions}

In this section we will try to analyze the structure of bi-distributions $w \in$ $(\mathcal{D}(\mathcal{T}) \otimes \mathcal{D}(\mathcal{T}))^{\prime}$ which are invariant under the left quasi-regular action $U_{g}^{\mathcal{T}}$ of $G$ on $\mathcal{D}(\mathcal{T})$

$$
w\left(U_{g}^{\mathcal{T}} f, U_{g}^{\mathcal{T}} h\right)=w(f, h), \forall f, h \in \mathcal{D}(\mathcal{T}),
$$

and compare with results obtained earlier in the literature.

Decomposing each $f=\sum f^{i} Y_{i}, f^{i} \in C_{0}^{\infty}(\Sigma)$, we find for $u \in \mathcal{D}(\mathcal{T})^{\prime}$ and $w \in$ $(\mathcal{D}(\mathcal{T}) \otimes \mathcal{D}(\mathcal{T}))^{\prime}$

$$
\begin{gathered}
u(f)=\sum_{i=1}^{n} u^{i}\left(f^{i}\right), w(f, h)=\sum_{i, j=1}^{n} w^{i j}\left(f^{i}, h^{j}\right), \\
u^{i} \in C_{0}^{\infty}(\Sigma)^{\prime}, w^{i j} \in\left(C_{0}^{\infty}(\Sigma) \otimes C_{0}^{\infty}(\Sigma)\right)^{\prime},
\end{gathered}
$$

so that the problem reduces to that for scalar distributions.

The following proposition establishes the general form of the $G$-invariant (or homogeneous) bi-distributions. Our approach is greatly inspired by [6] where this analysis is performed for $\mathbb{R}^{n}$.

Proposition 5.1. Every $w \in\left(C_{0}^{\infty}(\Sigma) \otimes C_{0}^{\infty}(\Sigma)\right)^{\prime}$ satisfying $w\left(U_{g} f, U_{g} h\right)=$ $w(f, h), \forall f, h \in C_{0}^{\infty}(\Sigma), g \in G$, has the form

$$
w(f, h)=u_{w}\left(\bar{f}^{*} * h\right)
$$

for some $u_{w} \in C_{0}^{\infty}(\Sigma)^{\prime}$. And conversely, any $u_{w} \in C_{0}^{\infty}(\Sigma)^{\prime}$ gives rise to such an invariant bi-distribution $w$.

Proof. Recall that for scalar functions $U_{g} f\left(x_{\Sigma} O\right)=f\left(g^{-1} x_{\Sigma} O\right)$. By the nuclear theorem $w$ can be uniquely extended to $\tilde{w} \in C_{0}^{\infty}(\Sigma \times \Sigma)^{\prime}$ via embedding

$$
C_{0}^{\infty}(\Sigma) \otimes C_{0}^{\infty}(\Sigma) \ni f\left(x_{\Sigma}\right) \otimes h\left(y_{\Sigma}\right) \rightarrow f\left(x_{\Sigma}\right) h\left(y_{\Sigma}\right) \in C_{0}^{\infty}(G \times G) .
$$

That

$$
w\left(f\left(g^{-1} x_{\Sigma} O\right), h\left(g^{-1} y_{\Sigma} O\right)\right)=w(f, h)
$$

by continuity implies that

$$
\tilde{w}\left(\phi\left(g^{-1} x_{\Sigma} O, g^{-1} y_{\Sigma} O\right)\right)=\tilde{w}\left(\phi\left(x_{\Sigma}, y_{\Sigma}\right)\right), \forall \phi \in C_{0}^{\infty}(\Sigma \times \Sigma) .
$$


Define the linear automorphism

$$
C_{0}^{\infty}(\Sigma \times \Sigma) \ni \phi\left(x_{\Sigma}, y_{\Sigma}\right) \rightarrow \psi_{\phi}\left(x_{\Sigma}, y_{\Sigma}\right) \in C_{0}^{\infty}(\Sigma \times \Sigma)
$$

by

$$
\psi_{\phi}\left(x_{\Sigma}, y_{\Sigma}\right)=\int_{O} d x_{O} \phi\left(x_{\Sigma}, x_{\Sigma} x_{O} y_{\Sigma} O\right)=\int_{O} d x_{O} \phi\left(x_{\Sigma}, x_{\Sigma} O x_{O} y_{\Sigma} O\right),
$$

and the pullback of $\tilde{w}$ under this automorphism by $\tilde{v}, \tilde{v}\left(\psi_{\phi}\right)=\tilde{w}(\phi)$. If $\phi_{g}\left(x_{\Sigma}, y_{\Sigma}\right)=$ $\phi\left(g^{-1} x_{\Sigma} O, g^{-1} y_{\Sigma} O\right)$ then

$$
\psi_{\phi_{g}}\left(x_{\Sigma}, y_{\Sigma}\right)=\int_{O} d x_{O} \phi\left(g^{-1} x_{\Sigma} O, g^{-1} x_{\Sigma} O x_{O} y_{\Sigma} O\right)=\psi_{\phi}\left(g^{-1} x_{\Sigma} O, y_{\Sigma}\right)
$$

Now

$$
\tilde{w}\left(\phi_{g}\right)=\tilde{v}\left(\psi_{\phi_{g}}\right)=\tilde{v}\left(\psi_{\phi}\right)=\tilde{w}(\phi)
$$

thus

$$
\tilde{v}\left(\psi_{\phi}\left(x_{\Sigma}, y_{\Sigma}\right)\right)=\tilde{v}\left(\psi_{\phi}\left(g^{-1} x_{\Sigma} O, y_{\Sigma}\right)\right), \forall g \in G
$$

Consider the restriction $v$ of $\tilde{v}$ to $C_{0}^{\infty}(\Sigma) \otimes C_{0}^{\infty}(\Sigma)$. The last equation implies $v\left(f\left(g^{-1} x_{\Sigma} O\right), h\left(y_{\Sigma}\right)\right)=v(f, h), \forall f, h \in C_{0}^{\infty}(\Sigma)$. If we fix $h$, then $v(., h) \in C_{0}^{\infty}(\Sigma)^{\prime}$ is a distribution which is invariant under all translations, and is thus given by a constant kernel, $v(f, h)=u_{w}(h) \int_{\Sigma} d x_{\Sigma} f\left(x_{\Sigma}\right)$, for some $u_{w}: C_{0}^{\infty}(\Sigma) \rightarrow \mathbb{C}$. On the other hand, if we fix $f$, then continuity in $h$ implies $u_{w} \in C_{0}^{\infty}(\Sigma)^{\prime}$. Because the integral $\int_{\Sigma} d x_{\Sigma} f\left(x_{\Sigma}\right)$ runs over a compact region, it can be transferred into $u_{w}$, i.e., $v(f, h)=u_{w}\left(\int_{\Sigma} d x_{\Sigma} f\left(x_{\Sigma}\right) h\left(y_{\Sigma}\right)\right)$. This in turn implies by continuity, that $\tilde{v}\left(\psi\left(x_{\Sigma}, y_{\Sigma}\right)\right)=u_{w}\left(\int_{\Sigma} d x_{\Sigma} \psi\left(x_{\Sigma}, y_{\Sigma}\right)\right)$. Finally we arrive at

$$
\begin{aligned}
& w(f, h)=\tilde{w}\left(f\left(x_{\Sigma}\right) h\left(y_{\Sigma}\right)\right)=\tilde{v}\left(f\left(x_{\Sigma}\right) \int_{O} d x_{O} h\left(x_{\Sigma} x_{o} y_{\Sigma} O\right)\right)= \\
& =u_{w}\left(\int_{\Sigma} d x_{\Sigma} f\left(x_{\Sigma}\right) \int_{O} d x_{O} h\left(x_{\Sigma} x_{O} y_{\Sigma} O\right)\right)=u_{w}\left(\bar{f}^{*} * h\right) .
\end{aligned}
$$

The converse statement is obvious.

For a distribution $w \in(\mathcal{D}(\mathcal{T}) \otimes \mathcal{D}(\mathcal{T}))^{\prime}$ this will mean

$$
w(f, h)=\sum_{i, j=1}^{n} u_{w}^{i j}\left(\left(\bar{f}^{i}\right)^{*} * h^{j}\right) .
$$

Remark 5.1. Note that every $G$-invariant bi-distribution $w \in\left(C_{0}^{\infty}(\Sigma) \otimes C_{0}^{\infty}(\Sigma)\right)^{\prime}$ is in particular $\Sigma$-invariant. Let $f * h(f \star h)$ and $f^{*}\left(f^{\star}\right)$ denote the convolution and the involution with respect to $G(\Sigma)$, respectively. Then

$$
w(f, h)=u_{w}\left(\int_{\Sigma} d x_{\Sigma} f\left(x_{\Sigma}\right) \int_{O} d x_{O} h\left(x_{\Sigma} x_{O} y_{\Sigma} O\right)\right)=
$$




$$
=u_{w}\left(\int_{O} d x_{O} L_{x_{O}^{-1}} \bar{f}^{\star} \star h\left(y_{\Sigma} O\right)\right)=u_{w}^{\prime}\left(\bar{f}^{\star} \star h\right)
$$

for some other $u_{w}^{\prime} \in\left(C_{0}^{\infty}(\Sigma) \otimes C_{0}^{\infty}(\Sigma)\right)^{\prime}$ as expected.

Let $\hat{\mathcal{D}}\left(\hat{G}_{\Sigma}\right)$ be the image of $C_{0}^{\infty}(\Sigma)$ under the harmonic analytical Fourier transform $f\left(x_{\Sigma}\right) \rightarrow \hat{f}(\pi)$. As an obvious corollary we arrive at the form of an invariant bi-distribution in the Fourier space.

Corollary 5.1. A G-invariant bi-distribution $w \in\left(C_{0}^{\infty}(\Sigma) \otimes C_{0}^{\infty}(\Sigma)\right)^{\prime}$ in the Fourier space is given by

$$
w(f, h)=\hat{u}_{w}\left(\pi\left(\bar{f}^{*}\right) \hat{h}(\pi)\right)=\hat{u}_{w}\left(\pi(\bar{f})^{*} \hat{h}(\pi)\right)
$$

for some $\hat{u}_{w} \in \hat{\mathcal{D}}\left(\hat{G}_{\Sigma}\right)^{\prime}$.

An immediate consequence of Proposition 4.2 is the following

Corollary 5.2. Under the assumptions of Proposition 4.2, a G-invariant bidistribution $w_{K} \in\left(C_{0}^{\infty}(K) \otimes C_{0}^{\infty}(K)\right)^{\prime}$ with $K \subset \Sigma$ compact is given by

$$
w_{K}(f, h)=\int_{\hat{G}_{\Sigma}} d \mu(\pi) \operatorname{Tr}\left[\hat{u}_{K}(\pi)^{*} \pi(\bar{f})^{*} \hat{h}(\pi)\right] .
$$

Proof. It suffices to note that

$$
\operatorname{supp}\{f * h\} \subset O(\operatorname{supp}\{f\})^{-1} \operatorname{supp}\{h\} O,
$$

and to apply Proposition 4.2 .

Finally we establish a generalization of the results by 5 for FRW spacetimes.

Proposition 5.2. Suppose that the group $G$ is such that all multiplicities $\operatorname{mult}\left(\pi, U_{g}\right)$ are finite. Then any $G$-invariant bi-distribution $w \in(D(\mathcal{T}) \otimes D(\mathcal{T}))^{\prime}$ has the form

$$
w(f, h)=\int_{\hat{G}_{\Sigma}} d \mu(\pi) \operatorname{Tr}\left[(\hat{\bar{f}}(\pi) \hat{u}(\pi))^{*} \hat{h}(\pi)\right],
$$

where $\hat{u}(\pi)$ is a $\mu$-locally measurable field of $\left[\operatorname{mult}\left(\pi, U_{g}\right) \cdot n\right] \times\left[\operatorname{mult}\left(\pi, U_{g}\right) \cdot n\right]$ complex matrices.

Proof. Let start with the case $w \in\left(C_{0}^{\infty}(\Sigma) \otimes C_{0}^{\infty}(\Sigma)\right)^{\prime}$. The condition that the modular function of $\Sigma$ has a nontrivial kernel ensures that the formula (7.49) of (Folland) is valid, so that for $\mu$-almost all $\pi$ the operator $D_{\pi}$ is invertible (injective). Therefore we can write $\pi(f)=\hat{f}(\pi) D_{\pi}^{-1}$, so that $\pi(\bar{f})^{*} \hat{h}(\pi)=D_{\pi}^{-1} \hat{\bar{f}}(\pi)^{*} \hat{h}(\pi)$ where $\hat{\bar{f}}(\pi)^{*} \hat{h}(\pi)$ is a $\operatorname{mult}\left(\pi, U_{g}\right) \times \operatorname{mult}\left(\pi, U_{g}\right)$ complex matrix. Now for any compact $K \subset \Sigma$ by Corollary 5.2 we find that the restriction $w_{K}$ of $w$ to $C_{0}^{\infty}(K) \otimes C_{0}^{\infty}(K)$ is given by

$w_{K}(f, h)=\int_{\hat{G}_{\Sigma}} d \mu(\pi) \operatorname{Tr}\left[\hat{u}_{K}^{\prime}(\pi)^{*} D_{\pi}^{-1} \hat{\bar{f}}(\pi)^{*} \hat{h}(\pi)\right]=\int_{\hat{G}_{\Sigma}} d \mu(\pi) \operatorname{Tr}\left[\hat{u}_{K}(\pi)^{*} \hat{\bar{f}}(\pi)^{*} \hat{h}(\pi)\right]$, 
where $\hat{u}_{K}(\pi)$ is a $\operatorname{mult}\left(\pi, U_{g}\right) \times \operatorname{mult}\left(\pi, U_{g}\right)$ complex matrix. Choosing a larger compact $K \subset K^{\prime} \subset \Sigma$ we will arrive at another matrix $\hat{u}_{K^{\prime}}(\pi)$. But when restricted to $K, w_{K^{\prime}}$ must coincide with $w_{K}$, hence $\hat{u}_{K^{\prime}}(\pi)=\hat{u}_{K}(\pi)$. Thus the matrix $\hat{u}_{K}(\pi)$ is the same for any $K$, and the formula holds for the entire $w$.

Now for $w \in(D(\mathcal{T}) \otimes D(\mathcal{T}))^{\prime}$ we have

$w(f, h)=\int_{\hat{G}_{\Sigma}} d \mu(\pi) \sum_{i, j=1}^{n} \operatorname{Tr}\left[\left(\hat{\bar{f}}^{i}(\pi) \hat{u}^{i j}(\pi)\right)^{*} \hat{h}^{j}(\pi)\right]=\int_{\hat{G}_{\Sigma}} d \mu(\pi) \operatorname{Tr}\left[(\hat{\hat{f}}(\pi) \hat{u}(\pi))^{*} \hat{h}(\pi)\right]$,

which completes the proof.

In the case of FRW spacetimes all the assumptions of the last proposition are satisfied. In particular all $\operatorname{mult}\left(\pi, U_{g}\right)=1$ and for the scalar case we find that any $G$-invariant bi-distribution is given by a locally measurable scalar field $\hat{u}(\pi)$.

\section{Acknowledgements}

The author expresses his thank to the Institute for Mathematics in the Sciences and in particular to the International Max Planck Research School for the organizational and material support of the $\mathrm{PhD}$ project which this work is a part of. Invaluable is the scientific patronage of the supervisor of this PhD project Prof. Dr. Rainer Verch, whose remarks and suggestions used in this work are too many to be listed here. Special gratitude is addressed to Prof. Dr. Gerald Folland for extremely helpful e-mail correspondence.

\section{Appendix A. Space structures. Distributions}

Let us start with introducing symmetric metric products

$$
\begin{aligned}
& \langle f, h\rangle_{M}=\int_{M} d \mu_{g}(x)\langle f(x), h(x)\rangle_{g}, f \in \mathcal{E}(\mathcal{T}), h \in \mathcal{D}(\mathcal{T}), \\
& \langle f, h\rangle_{\Sigma_{t}}=\int_{\Sigma} d \mu_{h}(\vec{x})\langle f(\vec{x}), h(\vec{x})\rangle_{g}, f \in \mathcal{E}\left(\mathcal{T}_{t}\right), h \in \mathcal{D}\left(\mathcal{T}_{t}\right) .
\end{aligned}
$$

The pseudo-Riemannian metric $\langle,\rangle_{\mathfrak{g}}$ induces a Krein space structure on $V$, the typical fiber of $\mathcal{T}$. Whence there is a Krein involution $\check{\Gamma}$, such that $(u, v)_{\mathfrak{g}}=\langle\bar{u}, \check{\Gamma} v\rangle_{\mathfrak{g}}$, $u, v \in V$, is a positive definite hermitian inner product. This gives rise to positive definite hermitian inner products

$$
\begin{aligned}
& (f, h)_{M}=\int_{M} d \mu_{g}(x)(f(x), h(x))_{\mathfrak{g}}, f \in \mathcal{E}(\mathcal{T}), h \in \mathcal{D}(\mathcal{T}), \\
& (f, h)_{\Sigma_{t}}=\int_{\Sigma} d \mu_{h}(\vec{x})(f(\vec{x}), h(\vec{x}))_{\mathfrak{g}}, f \in \mathcal{E}\left(\mathcal{T}_{t}\right), h \in \mathcal{D}\left(\mathcal{T}_{t}\right) .
\end{aligned}
$$

The completion of spaces $\mathcal{D}(\mathcal{T})$ and $\mathcal{D}\left(\mathcal{T}_{t}\right)$ with respect to these products becomes the Hilbert spaces $L^{2}(\mathcal{T})$ and $L^{2}\left(\mathcal{T}_{t}\right)$, respectively. The tangent space $T_{p} M$ at a 
point $p \in M$ with the Lorentzian metric $g$ is another example of a Krein space. In the same spirit one defines the positive definite inner product $(,)_{g}$ on $T_{p} M$. The metric $h$ on $T_{p} \Sigma$ is Riemannian, so the construction of $(,)_{h}$ is straightforward. Note that $\langle,\rangle_{\mathfrak{g}}$ and $g$ together give pseudo-Riemannian metrics on all product bundles $T^{*} M \otimes \ldots \otimes T^{*} M \otimes \mathcal{T}$ (respectively, $\langle,\rangle_{\mathfrak{g}}$ and $h$ on $T^{*} \Sigma \otimes \ldots \otimes T^{*} \Sigma \otimes \mathcal{T}_{t}$ ). All the resulting standard fibers are again Krein spaces, and can be given inner products $(,)_{g}$ in the same fashion. These in their turn produce products $(,)_{M}$ and $(,)_{\Sigma_{t}}$ on the respective sections.

The perfect countably Banach topology of the test function spaces $\mathcal{D}(\mathcal{T})$ and $\mathcal{D}\left(\mathcal{T}_{t}\right)$ can be given as usual (e.g., [7]). However, as we are going to perform a spectral analysis, we will need nuclear countably Hilbert space structure, to which we proceed [13]. Let $\mathcal{O} \subset M$ be a compact region. Let

$$
\mathcal{D}_{\mathcal{O}}(\mathcal{T})=\{f \in \mathcal{D}(\mathcal{T}): \operatorname{supp}\{f\} \subset \mathcal{O}\}
$$

and define the family of positive definite inner products $(,)_{\mathcal{O}, p}$ on $\mathcal{D}_{\mathcal{O}}(\mathcal{T})$ by

$$
(f, h)_{\mathcal{O}, p}=\sum_{q \leq p}\left((\nabla)^{q} f,(\nabla)^{q} h\right)_{M}, \forall f, h \in \mathcal{D}_{\mathcal{O}}(\mathcal{T}), p, q \in \mathbb{N}
$$

which induces a family of norms $\|\cdot\|_{\mathcal{O}, p}$. One can show that this family of norms is growing and consistent, and gives the same topology as the usual one. Let us give $\mathcal{D}_{\mathcal{O}}(\mathcal{T})$ a countably Hilbert space structure in the following sense,

$$
\mathcal{D}_{\mathcal{O}}(\mathcal{T})=\bigcap_{\mathbb{N}}{\overline{\mathcal{D}_{\mathcal{O}}(\mathcal{T})}}^{(,)_{\mathcal{O}, p}}
$$

It can be shown, that thus constructed countably Hilbert space $\mathcal{D}_{\mathcal{O}}(\mathcal{T})$ is nuclear. Let now

$$
\mathcal{O}_{1} \subset \ldots \subset \mathcal{O}_{n} \subset \ldots \subset M
$$

be an infinite family of growing compact regions. Then give $\mathcal{D}(\mathcal{T})$ the inductive limit topology

$$
\mathcal{D}(\mathcal{T})=\lim _{n \rightarrow \infty} \mathcal{D}_{\mathcal{O}_{n}}(\mathcal{T})
$$

Here we are done. Distributions $\mathcal{D}(\mathcal{T})^{\prime}$ and operations on them can be defined as usual. The same construction can be done for $\mathcal{D}\left(\mathcal{T}_{t}\right)$ with minor modifications.

At the end let us consider the choice of the topology in detail. In the literature one usually chooses the family of norms $\|\cdot\|_{p}$ (or sometimes a family of seminorms $|(.)|_{p}$; from these seminorms one can make norms by $\|\cdot\|_{p}=\sum_{q<p}|(\cdot)|_{q}$ or $\|\cdot\|_{p}=$ $\sup _{q<p}|(.)|_{q}$ etc.) rather arbitrarily in accordance with the setup of the problem, and it is tacitly assumed but not everywhere proven, that all such choices give equivalent topologies. Let us for consistency present here a proof of this fact. The zest of the proof (the usage of the Sobolev embedding theorem) was suggested by G. Folland.

Proposition Appendix A.1. Let $\mathcal{T} \stackrel{\pi}{\rightarrow} M$ be an $n$ dimensional pseudoRiemannian vector bundle over the d-dimensional parallelizable pseudo-Riemannian 
manifold $M$ with positive metric product $(,)_{\mathfrak{g}}$ constructed as above, so that we have well defined $L^{m}$ norms \|\|$\cdot\|\|_{m}$ for $1 \leq m \leq \infty$ on $\mathcal{D}(\mathcal{T})$. Let $\nabla$ be a connection on T. Let

(i) $X_{1} \ldots X_{d}$ be a system of first order smooth differential operators on $C^{\infty}(\mathcal{T})$ which span the tangent space $T^{*} M$ everywhere

(ii) the seminorms be given by $|(f)|_{\alpha, q}=\mid\left\|P_{\alpha, q}\left(X_{i}\right) f\right\| \|_{m}$, where $P_{\alpha, q}\left(X_{i}\right)$ are various monomials of order $q$ in $\left\{X_{i}\right\}, f \in \mathcal{D}(\mathcal{T})$

(iii) the family of norms be given as $\|f\|_{p}=\mid\left\|\left\{|(f)|_{\alpha, q}\right\}_{q \leq p}\right\| \|_{l^{k}}$, or by a superposition of different $|\|\cdot\||_{l^{k}}, 1 \leq k \leq \infty$.

Then the topology of $\mathcal{D}(\mathcal{T})$ defined by this family of norms is independent of the decisions (i) to (iii).

Proof. For convenience denote by $\left(X_{i}, m, *\right)$ the triple of choices at points (i),(ii) and (iii). Then $\left(X_{i}, m, *\right) \sim\left(X_{i}^{\prime}, m^{\prime}, *^{\prime}\right)$ will mean that this two topologies are equivalent.

As the topology of $\mathcal{D}(\mathcal{T})$ is the inductive limit of various $\mathcal{D}\left(\mathcal{T}_{K}\right)$ with $\mathcal{T}_{K}=$ $\pi^{-1}(K), K \subset M$ compact, it suffices to prove the assertion for an arbitrary $\mathcal{D}\left(\mathcal{T}_{K}\right)$. The topologies given by two families of norms $\left\{\|\cdot\|_{p}\right\}$ and $\left\{\|\cdot\|_{p}^{\prime}\right\}$ are equivalent if and only if these two systems of norms are themselves equivalent, i.e., $\forall p, \exists q(p), r(p)>0$, $0<C_{p}, C_{p}^{\prime} \in \mathbb{R}$ such that $\|\cdot\|_{p} \leq C_{p}\|\cdot\|_{q(p)}$ and $\|\cdot\|_{p}^{\prime} \leq C_{p}^{\prime}\left\|_{\cdot}\right\|_{r(p)}^{\prime}$. Let us start with the point (iii). Suppose the choices (i) and (ii) are fixed, i.e., consider $\left(X_{i}, m, *\right)$ and $\left(X_{i}, m, *^{\prime}\right)$. Then all possible choices in (iii) give equivalent systems of norms because of the elementary inequalities

$\left|\left\|\left\{|(f)|_{\alpha, q}\right\}_{I}\right\|\right|_{l^{\infty}} \leq \ldots \leq\left.\left|\left\|\left\{|(f)|_{\alpha, q}\right\}_{I}\right\|_{l^{k}} \leq \ldots \leq\right|\left\|\left\{|(f)|_{\alpha, q}\right\}_{I}\right\|\right|_{l^{1}} \leq N_{I} \mid\left\|\left\{|(f)|_{\alpha, q}\right\}_{I}\right\| \|_{l^{\infty}}$,

where $N_{I}$ is the number of terms in the index set $I$. These inequalities can be applied consecutively to estimate any composite norm by, say, $|\|\cdot\||_{l_{\infty}}$. An example of a composite norm is $\|f\|_{p}=\sup _{q \leq p} \mid\left\|\nabla^{q} f\right\|_{\infty}$. We found that $\left(X_{i}, m, *\right) \sim\left(X_{i}, m, *^{\prime}\right)$.

Now let $1 \leq m \leq \infty$ at (ii) and $k=\infty$ at (iii) be chosen, and choose two systems of operators $\left\{X_{i}\right\}$ and $\left\{Y_{i}\right\}$ at point (i) to construct the families of norms $\left\{\|\cdot\|_{p}\right\}$ and $\left\{\|\cdot\|_{p}^{\prime}\right\}$, respectively. This corresponds to $\left(X_{i}, m, l^{\infty}\right)$ and $\left(Y_{i}, m, l^{\infty}\right)$. Because $\left\{X_{i}\right\}$ spans $T^{*} M$, there are functions $c_{i j}(x) \in C^{\infty}(M)$ and smooth fields of homomorphisms $\tilde{\Gamma}_{i} \in C^{\infty}(H o m(\mathcal{T}, \mathcal{T}))$ with $Y_{i}(x)=\sum_{j} c_{i j}(x) X_{j}(x)+\tilde{\Gamma}_{i}$. Using this for any monomial $P_{\alpha, q}\left(Y_{i}\right)$ we get

$$
P_{\alpha, q}\left(Y_{i}\right) f=\sum_{\beta} c_{\alpha, q}^{\beta}(x) Q_{\alpha, q}^{\beta}\left(X_{i}\right) f
$$

where $c_{\alpha, q}^{\beta}(x) \in C^{\infty}(M)$ and $Q_{\alpha, q}^{\beta}\left(X_{i}\right)$ are monomials of order less or equal $q$. The number of summands is less than, say, $(4 d)^{q}$. It follows by Minkowsky inequality

$$
|(f)|_{\alpha, q}^{\prime}=\left|\left\|P_{\alpha, q}\left(Y_{i}\right) f\right\|\right|_{m} \leq \sum_{\beta} \mid\left\|c_{\alpha, q}^{\beta}(x) Q_{\alpha, q}^{\beta}\left(X_{i}\right) f\right\|_{m},
$$


and then by Hölder inequality

$\left.\sum_{\beta}\left|\left\|c_{\alpha, q}^{\beta}(x) Q_{\alpha, q}^{\beta}\left(X_{i}\right) f\right\|_{m} \leq C_{\alpha, q} \sum_{\beta}\right|\left\|Q_{\alpha, q}^{\beta}\left(X_{i}\right) f\right\|\right|_{m}=C_{\alpha, q} \sum_{\beta}|(f)|_{\alpha(\alpha, q, \beta), q(\alpha, q, \beta)}$,

where $0<C_{\alpha, q}=\sup _{\beta}\left|\left\|c_{\alpha, q}^{\beta}\right\|\right|_{\infty}$. In other words, the seminorms of order $q$ of the second system can be estimated by linear combinations of seminorms of the first system of the same or lower order. Then

$$
\begin{gathered}
\|f\|_{p}^{\prime}=\sup _{q \leq p}|(f)|_{\alpha, q}^{\prime} \leq C_{p} \sup _{q \leq p} \sum_{\beta}|(f)|_{\alpha(\alpha, q, \beta), q(\alpha, q, \beta)} \leq \\
\leq C_{p}(4 d)^{p} \sup _{q \leq p}|(f)|_{\alpha(\alpha, q, \beta), q(\alpha, q, \beta)} \leq C_{p}(4 d)^{p} \sup _{q \leq p}|(f)|_{\alpha, q}=C_{p}(4 d)^{p}\|f\|_{p},
\end{gathered}
$$

where $0<C_{p}=\sup _{q \leq p} C_{\alpha, q}$. For the other direction of the estimate we simply need to switch $\left\{X_{i}\right\}$ and $\left\{Y_{i}\right\}$. Thus these two topologies are equivalent, $\left(X_{i}, m, l^{\infty}\right) \sim$ $\left(Y_{i}, m, l^{\infty}\right)$.

Finally let $X_{i}=\nabla_{i}$ (components with respect to a global orthonromal frame in $T^{*} M$ ) be chosen at (i), and $\|\cdot\|=\mid\left\|\left\{|(.)|_{\alpha, q}\right\}_{q \leq p}\right\| \|_{l^{2}}$ at (iii). We construct two families of norms by choosing $1 \leq m<\infty$ and $m^{\prime}=\infty$ at (ii) for $\|\cdot\|_{p}$ and $\|\cdot\|_{p}^{\prime}$, respectively. This can be symbolized as $\left(\nabla_{i}, m, l^{2}\right)$ and $\left(\nabla_{i}, \infty, l^{2}\right)$. Because $K$ is compact, by an application of Hölder inequality we obtain

$$
|\|\cdot\||_{m} \leq C_{m}|\|\cdot\||_{\infty}
$$

for some $0<C_{m} \in \mathbb{R}$, and hence obviously

$$
\|\cdot\|_{p} \leq C_{m}\|\cdot\|_{p}^{\prime}, p \in \mathbb{N}_{0} .
$$

The opposite inequality requires an application of Sobolev embedding theorem for compact manifolds [25, [26]. Denote the Sobolev norms (which are equivalent to those in [25])

$$
\mid\|f\|_{W^{p, m}}=\sqrt{\sum_{q \leq p}\left|\left\|\nabla^{q} f\right\|\right|_{m}^{2}} .
$$

Then an application of Sobolev embedding theorem gives

$$
|\|\cdot\||_{W^{0, \infty}}=|\|\cdot\||_{\infty} \leq D|\|\cdot\||_{W^{d, 1}}
$$

for some $0<D \in \mathbb{R}$. By another application of Hölder inequality we find

$$
\|\| \cdot\left\|\left.\right|_{W^{d, 1}} \leq|\|\cdot\||_{W^{d, 2}}\right.
$$

and therefore

$$
\|\| \cdot \|\left.\right|_{\infty} \leq D \sqrt{\sum_{q \leq d}\left|\left\|\nabla^{q} f\right\|\right|_{2}^{2}}
$$

Next

$$
\left\|\nabla^{q} f\right\|_{2}^{2}=\sum_{\alpha} \mid\left\|P_{\alpha, q}\left(X_{i}\right) f\right\|_{2}^{2}
$$


and finally

$$
\begin{gathered}
\|f\|_{p}^{\prime}=\sqrt{\sum_{q \leq p}\left|\left\|P_{\alpha, q}\left(X_{i}\right) f\right\|\right|_{\infty}^{2}} \leq D \sqrt{\sum_{q \leq p} \sum_{j \leq d} \mid\left\|\nabla^{j} P_{\alpha, q}\left(X_{i}\right) f\right\|_{2}^{2}}= \\
=D \sqrt{\sum_{q \leq p} \sum_{j \leq d}\left|\left\|P_{\beta, j}\left(X_{i}\right) P_{\alpha, q}\left(X_{i}\right) f\right\|\right|_{2}^{2}} \leq D \sqrt{\sum_{q \leq p+d}\left\|P_{\alpha, q}\left(X_{i}\right) f\right\|_{2}^{2}} \leq \\
\leq D E \sqrt{\sum_{q \leq p+d} \mid\left\|P_{\alpha, q}\left(X_{i}\right) f\right\|_{m}^{2}}=D E\|f\|_{p+d}^{\prime},
\end{gathered}
$$

where in the last inequality again Hölders inequality was used with some $0<E \in \mathbb{R}$. Thus we have shown that choosing any $1 \leq m<\infty$ is equivalent to choosing $m=\infty$ at point (ii), i.e., $\left(\nabla_{i}, m, l^{2}\right) \sim\left(\nabla_{i}, \infty, l^{2}\right)$.

Write

$$
\begin{gathered}
\left(X_{i}, m, *\right) \sim\left(X_{i}, m, l^{\infty}\right) \sim\left(\nabla_{i}, m, l^{\infty}\right) \sim\left(\nabla_{i}, m, l^{2}\right) \sim\left(\nabla_{i}, \infty, l^{2}\right) \sim\left(\nabla_{i}, m^{\prime}, l^{2}\right) \sim \\
\sim\left(\nabla_{i}, m^{\prime}, l^{\infty}\right) \sim\left(X_{i}^{\prime}, m^{\prime}, l^{\infty}\right) \sim\left(X_{i}^{\prime}, m^{\prime}, *^{\prime}\right) .
\end{gathered}
$$

The proof is complete.

\section{Appendix B. On the time dependent harmonic oscillator}

Here we will concentrate on some properties of the solutions of the smooth complex time dependent harmonic oscillator equation

$$
\ddot{T}(s)+\Lambda(s) T(s)=0
$$

where $\Lambda(s)$ is a smooth complex function on the real line. This equation is under attention since a long time, but some results are not that easily available today (at least for us).

We start with an easy remark. Denote by

$$
W[Q, R](s)=\left(\begin{array}{cc}
Q(s) & \dot{Q}(s) \\
R(s) & \dot{R}(s)
\end{array}\right)
$$

the Wronski matrix of two solutions $Q$ and $R$.

Remark Appendix B.1. Let $Q, R$ be two linearly independent solutions of (Eq $\mathrm{B.1})$, and $T$ an arbitrary solution. Then from the conservation of $\operatorname{det} W[Q, T]$ and $\operatorname{det} W[R, T]$ it is easy to find

$$
\begin{gathered}
\left(\begin{array}{c}
\dot{T}(s) \\
-T(s)
\end{array}\right)=W[Q, R]^{-1}(s) \times W[Q, R](0) \times\left(\begin{array}{c}
\dot{T}(0) \\
-T(0)
\end{array}\right)= \\
=\operatorname{det} W[Q, R]^{-1}(0)\left(\begin{array}{cc}
\dot{R}(s) & -\dot{Q}(s) \\
-R(s) & Q(s)
\end{array}\right) \times\left(\begin{array}{cc}
Q(0) & \dot{Q}(0) \\
R(0) & \dot{R}(0)
\end{array}\right) \times\left(\begin{array}{c}
\dot{T}(0) \\
-T(0)
\end{array}\right) .
\end{gathered}
$$


Thus having at hand two such particular solutions $Q, R$, we have a control over arbitrary solutions $T$ in terms of their initial data.

Our first task is to obtain a control over the magnitude of the solution $T$ on a given compact interval $\mathcal{R}$ in terms of its initial data $T(0)$ and $\dot{T}(0)$. This is done by the so called energy estimate. Define the energy of a solution $T$ by

$$
\mathcal{W}[\breve{T}](s)=\frac{1}{2}|\dot{T}|^{2}(s)+\frac{1}{2} \Re \Lambda(s)|T(s)|^{2} .
$$

If $\Re \Lambda>0$ on $\mathcal{R}$ then $2 \mathcal{W}[\breve{T}]$ dominates $\Re \Lambda|T|^{2}$ and $|\dot{T}|^{2}$, and obtaining bounds on $\mathcal{W}[\breve{T}]$ we automatically get bounds on $|T|$ and $|\dot{T}|$.

Proposition Appendix B.1. For arbitrary solution $T$ of

$$
\ddot{T}(s)+\Lambda(s) T(s)=0,
$$

with smooth complex valued $\Lambda(s)$ having a positive real part (i.e., $\Re \Lambda(s)>0$ ) on a compact interval $\mathcal{R}$, the energy function $\mathcal{W}[\breve{T}](s)$ satisfies the estimate

$\mathcal{W}[\breve{T}](0) e^{-\int_{0}^{s} d \sigma\left(\frac{2|\Im \Lambda(\sigma)|}{\sqrt{\Re \Lambda(\sigma)}}+\left|\partial_{s} \ln \Re \Lambda(\sigma)\right|\right)} \leq \mathcal{W}[\breve{T}](s) \leq \mathcal{W}[\breve{T}](0) e^{\int_{0}^{s} d \sigma\left(\frac{2|\Im \Lambda(\sigma)|}{\sqrt{\Re \Lambda(\sigma)}}+\left|\partial_{s} \ln \Re \Lambda(\sigma)\right|\right)}$ for all $s \in \mathcal{R}$.

Proof. Write $T(s)=R(s)+i S(s), \Lambda(s)=\Theta(s)+i \Xi(s)$, and insert into the equation. We will get the following system of real equations,

$$
\left\{\begin{array}{l}
\ddot{R}(s)+\Theta(s) R(s)-\Xi(s) S(s)=0, \\
\ddot{S}(s)+\Theta(s) S(s)+\Xi(s) R(s)=0 .
\end{array}\right.
$$

We can cast this into a real vector equation

$$
\ddot{\breve{T}}(s)+\hat{\Lambda}(s) \breve{T}(s)=0
$$

by denoting

$$
\breve{T}(s)=(R(s), S(s))^{\top}
$$

and

$$
\hat{\Lambda}(s)=\left(\begin{array}{cc}
\Theta(s) & -\Xi(s) \\
\Xi(s) & \Theta(s)
\end{array}\right)=\hat{\Lambda}^{+}(s)+\hat{\Lambda}^{-}(s)=\Theta(s) \mathbf{1}+\Xi(s)\left(\begin{array}{cc}
0 & -1 \\
1 & 0
\end{array}\right),
$$

where $\hat{\Lambda}^{ \pm}$denote the symmetric and antisymmetric parts. The energy function equals

$$
\mathcal{W}[\breve{T}](s)=\frac{1}{2} \dot{\breve{T}}^{2}(s)+\frac{1}{2} \breve{T}^{\top}(s) \hat{\Lambda}(s) \breve{T}(s)=\frac{1}{2} \dot{\breve{T}}^{2}(s)+\frac{1}{2} \breve{T}^{\top}(s) \hat{\Lambda}^{+}(s) \breve{T}(s) .
$$

On the interval $\mathcal{R}$ we have $\mathcal{W}[\breve{T}](s)>0$ as by the assumption $\Theta(s)>0$. One can easily find that

$$
\dot{\mathcal{W}}[\breve{T}](s)=\breve{T}^{\top}(s) \hat{\Lambda}^{-}(s) \dot{T}(s)+\frac{1}{2} \breve{T}^{\top}(s) \dot{\hat{\Lambda}}^{+}(s) \breve{T}(s)
$$


whence it follows

$$
|\dot{\mathcal{W}}[\breve{T}](s)| \leq|\Xi(s)||\breve{T}(s)||\dot{\widetilde{T}}(s)|+\left|\partial_{s} \ln \Theta(s)\right| \mathcal{W}[\breve{T}](s) .
$$

By definition of $\mathcal{W}[\breve{T}]$ and positivity of $\Theta$ we have $|\dot{\widetilde{T}}(s)| \leq \sqrt{2 \mathcal{W}[\breve{T}](s)}$ and $|\breve{T}(s)| \leq$ $\sqrt{2 \mathcal{W}[\breve{T}](s) / \Theta(s)}$ on $\mathcal{R}$. It follows then

$$
\left|\partial_{s} \ln \mathcal{W}[\breve{T}](s)\right| \leq \frac{2|\Xi(s)|}{\sqrt{\Theta(s)}}+\left|\partial_{s} \ln \Theta(s)\right|,
$$

and integrating this we finally arrive at

$$
\mathcal{W}[\breve{T}](0) e^{-\left|\int_{0}^{s} d \sigma\left(\frac{2|\Xi(\sigma)|}{\sqrt{\Theta(\sigma)}}+\left|\partial_{s} \ln \Theta(\sigma)\right|\right)\right|} \leq \mathcal{W}[\breve{T}](s) \leq \mathcal{W}[\breve{T}](0) e^{\left|\int_{0}^{s} d \sigma\left(\frac{2|\Xi(\sigma)|}{\sqrt{\Theta(\sigma)}}+\left|\partial_{s} \ln \Theta(\sigma)\right|\right)\right|},
$$

precisely as in the statement.

If however $\Lambda$ is not guaranteed to be positive, then on those regions where it is negative the magnitude of the solutions is expected to behave exponentially. We are able to capture that exponential factor by the following beautiful trick.

Proposition Appendix B.2. For any $0<\kappa \in \mathbb{R}$, any solution of the equation

$$
\ddot{T}(s)+\Lambda(s) T(s)=0
$$

can be represented as $T(s)=\tau\left(\frac{1}{\kappa} \operatorname{th}(\kappa s)\right) \operatorname{ch}(\kappa s)$, where $\tau(z)$ is a solution of the equation

$$
\ddot{\tau}(z)+\Omega(z) \tau(z)=0
$$

with

$$
\Omega(z)=\frac{\kappa^{2}+\Lambda\left(\frac{1}{\kappa} \operatorname{ath}(\kappa z)\right)}{\left(1-\kappa^{2} z^{2}\right)^{2}}, z \in\left(-\frac{1}{\kappa}, \frac{1}{\kappa}\right)
$$

Proof. The proof is elementary once we already know the clue: the substitution of variables $\kappa z=\operatorname{th}(\kappa s)$. The substitution $T(s)=\tau(s) \operatorname{ch}(\kappa s)$ into the original equation gives

$$
\ddot{\tau}(s)+2 \kappa \operatorname{th}(\kappa s) \dot{\tau}(s)+\left(\kappa^{2}+\Lambda(s)\right) \tau(s)=0,
$$

then the substitution $s \rightarrow z$ yields the final formulas.

Let us say a couple of words about this. If $\Re \Lambda$ has a minimal negative value $-c$ on some domain, then it suffices to set $\kappa=\sqrt{c}$ to reduce the problem to an oscillatory equation for $\rho$. The upper bound of the rate of exponential expansion is precisely given by the square root of the minimal negative value of $\Re \Lambda$.

Finally we combine these two statements to find an explicit uniform bound on an arbitrary solution $T$. Let the compact interval $\mathcal{R}$ containing 0 be fixed, and set $A_{\mathcal{R}}=\sup _{\mathcal{R}}|\Im \Lambda|, c_{\mathcal{R}}=\inf _{\mathcal{R}} \Re \Lambda, \kappa=\sqrt{1+\left|\min \left\{0, c_{\mathcal{R}}\right\}\right|}, B_{\mathcal{R}}=\sup _{\mathcal{R}}\left|\partial_{s} \ln \left(\kappa^{2}+\Re \Lambda\right)\right|$, 


$$
\begin{gathered}
D_{\mathcal{R}}=\sup _{\mathcal{R}}\left(\kappa^{2}+\Re \Lambda\right), e_{\mathcal{R}}=\inf _{\mathcal{R}}\left(\kappa^{2}+\Re \Lambda\right)=1+\max \left\{0, c_{\mathcal{R}}\right\}, \\
L_{\mathcal{R}}=\left(2 A_{\mathcal{R}}+\operatorname{ch}^{2}(\kappa|\mathcal{R}|) B_{\mathcal{R}}+2 \kappa \operatorname{sh}(2 \kappa|\mathcal{R}|)\right)
\end{gathered}
$$

(we suppressed the index $\mathcal{R}$ of $\kappa$ for convenience).

Corollary Appendix B.1. For an arbitrary solution $T$ it holds

$$
|T(s)| \leq|T(0)| \sqrt{\frac{D_{\mathcal{R}}}{e_{\mathcal{R}}}} e^{L_{\mathcal{R}}} \operatorname{ch}(\kappa|\mathcal{R}|)+|\dot{T}(0)| \frac{1}{\sqrt{e_{\mathcal{R}}}} e^{L_{\mathcal{R}}} \operatorname{ch}(\kappa|\mathcal{R}|)
$$

for all $s \in \mathcal{R}$.

Proof. Consider the linearly independent solutions $Q$ and $R$ given by initial data

$$
Q(0)=1, \dot{Q}(0)=0, R(0)=0, \dot{R}(0)=1 .
$$

Using Proposition Appendix B.2 represent them as $Q(s)=\xi(z(s)) \operatorname{ch}(\kappa s)$ and $R(s)=\rho(z(s)) \operatorname{ch}(\kappa s)$, where $\xi(z)$ and $\rho(z)$ are solutions of the equation

$$
\ddot{\tau}(z)+\Omega(z) \tau(z)=0
$$

with

$$
\Omega(z)=\frac{\kappa^{2}+\Lambda\left(\frac{1}{\kappa} \operatorname{ath}(\kappa z)\right)}{\left(1-\kappa^{2} z^{2}\right)^{2}}
$$

Using $z(0)=0$ and

$$
\frac{d}{d s}[\tau(z(s)) \operatorname{ch}(\kappa s)]=\frac{\dot{\tau}(z(s))}{\operatorname{ch}(\kappa s)}+\tau(z(s)) \operatorname{sh}(\kappa s) \kappa,
$$

we find

$$
\xi(0)=1, \dot{\xi}(0)=0, \rho(0)=0, \dot{\rho}(0)=1 .
$$

Note that $\Re \Omega(s)=\kappa^{2}+\Re \Lambda \geq 1$, thus Proposition Appendix B.1 is applicable for $\xi$ and $\rho$. We have $\mathcal{W}[\xi](0)=\frac{1}{2} \Re \Omega(0)$ and $\mathcal{W}[\rho](0)=\frac{1}{2}$. Now

$$
\begin{gathered}
\frac{d}{d z} \ln \Re \Omega(z)=\frac{d s}{d z}(s) \frac{d}{d s} \ln \Re \Omega(z(s))=\operatorname{ch}^{2}(\kappa s) \frac{d}{d s} \ln \left(\left(\kappa^{2}+\Re \Lambda(s)\right) \operatorname{ch}^{4}(\kappa s)\right)= \\
=\operatorname{ch}^{2}(\kappa s) \frac{d}{d s} \ln \left(\kappa^{2}+\Re \Lambda(s)\right)+2 \kappa \operatorname{sh}(2 \kappa s) .
\end{gathered}
$$

Then it follows

$$
\begin{gathered}
\left|\int_{0}^{z} d \sigma\left(\frac{2|\Im \Omega(\sigma)|}{\sqrt{\Re \Omega(\sigma)}}+\left|\partial_{z} \ln \Re \Omega(\sigma)\right|\right)\right| \leq \frac{2}{\kappa} \operatorname{th}(\kappa|\mathcal{R}|)\left(2 A_{\mathcal{R}}+\operatorname{ch}^{2}(\kappa|\mathcal{R}|) B_{\mathcal{R}}+2 \kappa \operatorname{sh}(2 \kappa|\mathcal{R}|)\right) \leq \\
\leq 2\left(2 A_{\mathcal{R}}+\operatorname{ch}^{2}(\kappa|\mathcal{R}|) B_{\mathcal{R}}+2 \kappa \operatorname{sh}(2 \kappa|\mathcal{R}|)\right)=2 L_{\mathcal{R}} .
\end{gathered}
$$

By Proposition Appendix B.1 we have

$$
\mathcal{W}[\xi](z) \leq \mathcal{W}[\xi](0) e^{2 L_{\mathcal{R}}}, \mathcal{W}[\rho](z) \leq \mathcal{W}[\rho](0) e^{2 L_{\mathcal{R}}},
$$


which entails

$$
\begin{gathered}
|\xi(z)| \leq \sqrt{\frac{\kappa^{2}+\Re \Lambda(0)}{\kappa^{2}+\Re \Lambda(s(z))}} e^{L_{\mathcal{R}}},|\dot{\xi}(z)| \leq \sqrt{\kappa^{2}+\Re \Lambda(0)} e^{L_{\mathcal{R}}}, \\
|\rho(z)| \leq \frac{1}{\sqrt{\kappa^{2}+\Re \Lambda(s(z))}} e^{L_{\mathcal{R}}},|\dot{\rho}(z)| \leq e^{L_{\mathcal{R}}} .
\end{gathered}
$$

For $Q$ and $R$ we get

$$
|Q(s)| \leq \sqrt{\frac{D_{\mathcal{R}}}{e_{\mathcal{R}}}} e^{L_{\mathcal{R}}} \operatorname{ch}(\kappa|\mathcal{R}|),|R(s)| \leq \frac{1}{\sqrt{e_{\mathcal{R}}}} e^{L_{\mathcal{R}}} \operatorname{ch}(\kappa|\mathcal{R}|),
$$

and using (EqB.2)

$$
|\dot{Q}(s)| \leq \sqrt{D_{\mathcal{R}}} e^{L_{\mathcal{R}}}\left(1+\frac{\kappa \operatorname{sh}(\kappa|\mathcal{R}|)}{\sqrt{e_{\mathcal{R}}}}\right),|\dot{R}(s)| \leq e^{L_{\mathcal{R}}}\left(1+\frac{\kappa \operatorname{sh}(\kappa|\mathcal{R}|)}{\sqrt{e_{\mathcal{R}}}}\right) .
$$

Finally let $T$ be an arbitrary solution of the original equation. Applying Remark Appendix B.1 for $Q, R$ and $T$ we find

$$
T(s)=T(0) Q(s)+\dot{T}(0) R(s),
$$

and hence

$$
|T(s)| \leq|T(0)| \sqrt{\frac{D_{\mathcal{R}}}{e_{\mathcal{R}}}} e^{L_{\mathcal{R}}} \operatorname{ch}(\kappa|\mathcal{R}|)+|\dot{T}(0)| \frac{1}{\sqrt{e_{\mathcal{R}}}} e^{L_{\mathcal{R}}} \operatorname{ch}(\kappa|\mathcal{R}|),
$$

as asserted.

\section{Appendix C. A result from functional calculus}

In this section we will obtain a result using the theory of holomorphic functional calculus of strip type operators. We are grateful to M. Haase for very useful comments on this theory, and refer to his book [27] for all the information necessary in this section.

Let $\mathbb{H}_{a}=\{z \in \mathbb{C}:|\Im z|<a\}$ denote the symmetric strip of height $a>0$. If for an (unbounded) operator $A$ on the Banach space $\mathcal{X}$ we have $A \in \operatorname{Strip}(a)$, then we can apply the holomorphic functional calculus of $A$ given by

$$
F(A)=\frac{1}{2 \pi i} \int_{\gamma_{a}} d z f(z) \Re(z, A), \forall F \in \mathcal{M}\left[\mathbb{H}_{a}\right],
$$

where $\gamma_{a}=\partial \mathbb{H}_{a}$ oriented positively (counterclockwise), and $\mathfrak{R}(z, A)$ is the resolvent of $A$ for $z \in \mathbb{C}$. Define

$$
\mathcal{A}\left(\mathbb{H}_{a}\right)=\left\{F \in \operatorname{Hol}\left(\mathbb{H}_{a}\right): \exists N \in \mathbb{N} \text { s.t. } F=O\left(|\Re z|^{N}\right)\right\},
$$

and

$$
\mathcal{A}\left[\mathbb{H}_{a}\right]=\bigcup_{b>a} \mathcal{A}\left(\mathbb{H}_{b}\right) .
$$


Now let $D_{\Sigma_{t}}=-\Delta+m^{\star}(t, \vec{x})$ be the known real lower semi-bounded operator acting on the vector bundle $\mathcal{T}_{t}$ over a Riemannian manifold $\Sigma_{t}$, and let $K \subset \Sigma_{t}$ be a compact region. Denote

$$
\mathcal{D}(K)=\left\{f \in \mathcal{D}\left(\mathcal{T}_{t}\right): \operatorname{supp} f \subset K\right\}
$$

Then we have the following result.

Proposition Appendix C.1. For any $F \in \mathcal{A}\left[\mathbb{H}_{0}\right]$ and $f \in \mathcal{D}(K)$ it follows

$$
F\left(D_{\Sigma_{t}}\right) f \in \mathcal{D}(K) .
$$

Proof. Let the nuclear topology be given by $\left(X_{i}, 2, l^{2}\right)$, i.e., for any $p \in \mathbb{N}_{0}$ we set

$$
(f, h)_{p}=\sum_{q \leq p}\left(Q_{\alpha, q}\left(X_{i}\right) f, Q_{\alpha, q}\left(X_{i}\right) h\right)_{L^{2}}
$$

and consider the induced norms $\|\cdot\|_{p}$. Define the Hilbert spaces

$$
\mathcal{H}_{p}=\overline{\mathcal{D}(K)}^{(,)_{p}}
$$

then by the property of the countably normed spaces we have

$$
\begin{aligned}
& \mathcal{H}_{p} \subset \mathcal{H}_{q}, q<p, \\
& \mathcal{D}(K)=\bigcap_{p=0}^{\infty} \mathcal{H}_{p} .
\end{aligned}
$$

Fix $p$, and define the operator $D_{p}$ on $\mathcal{H}_{p}$ by setting $D_{p} f=D_{\Sigma_{t}} f$ for all $f \in$ $\operatorname{Dom}\left(D_{\Sigma_{t}}\right) \cap \mathcal{H}_{p}$, then $\operatorname{Dom}\left(D_{p}\right) \supset \mathcal{H}_{p+2}$ is a dense subspace of $\mathcal{H}_{p}$. Then $D_{p}$ is a real symmetric operator, and hence by von Neumann's theorem possesses a self-adjoint extension $A_{p}$ which needs not be lower semi-bounded. The self-adjoint operator $A_{p}$ has a purely real spectrum, thus $A_{p} \in \operatorname{Strip}(0)$. Let $\mathcal{A}\left(\mathbb{H}_{a}\right) \ni F(z)=O\left(|\Re z|^{N}\right)$, then for a sufficiently large $a<\lambda \in \mathbb{R}$, the function $e(z)=(z-i \lambda)^{-(N+2)}$ will regularize $F$ on $\mathbb{H}_{a}$. In particular, we will have $[e F]\left(A_{p}\right) \in \mathcal{B}\left(\mathcal{H}_{p}\right)$. Then $F\left(A_{p}\right)=\left(A_{p}-\right.$ $i \lambda)^{N+2}[e F]\left(A_{p}\right)=[e F]\left(A_{p}\right)\left(A_{p}-i \lambda\right)^{N+2}$, from where it follows that $\operatorname{Dom}\left(A_{p}^{N+2}\right) \subset$ $\operatorname{Dom}\left(F\left(A_{p}\right)\right)$. From the definition of $A_{p}$ it is clear that $\mathcal{H}_{p+2(N+2)} \subset \operatorname{Dom}\left(A_{p}^{N+2}\right)$, whence $\mathcal{H}_{p+2(N+2)} \subset \operatorname{Dom}\left(F\left(A_{p}\right)\right)$. Thus we have established, that whenever $f \in$ $\mathcal{H}_{p+2(N+2)}$, then necessarily $F\left(A_{p}\right) f \in \mathcal{H}_{p}$. Now if $f \in \mathcal{D}(K)$, then for any $p \geq 0$ we have $f \in \mathcal{H}_{p+2(N+2)}$, and hence $F\left(A_{p}\right) f \in \mathcal{H}_{p}$. Meanwhile for any $p \geq 0$, the self-adjoint operator $D_{\Sigma_{t}}$ agrees with $A_{p}$ on $\mathcal{D}(K)$. Therefore also their functional calculi agree, $F\left(D_{\Sigma_{t}}\right) f=F\left(A_{p}\right) f \in \mathcal{H}_{p}$. Thus

$$
F\left(D_{\Sigma_{t}}\right) f \in \bigcap_{p=0}^{\infty} \mathcal{H}_{p}=\mathcal{D}(K),
$$

which completes the proof. 


\section{Bibliography}

[1] A. Degner and R. Verch, "Cosmological particle creation in states of low energy," J. Math. Phys., vol. 51, no. 2, p. 022302, 2010.

[2] L. Parker, "Quantized Fields and Particle Creation in Expanding Universes. I," Phys. Rev., vol. 183, pp. 1057-1068, July 1969.

[3] S. A. Fulling, Aspects of Quantum Field Theory in Curved Spacetime (London Mathematical Society Student Texts). Cambridge University Press, 1989.

[4] C. J. Fewster and M. J. Pfenning, "A Quantum weak energy inequality for spin one fields in curved space-time," J.Math.Phys., vol. 44, pp. 4480-4513, 2003.

[5] C. Lüders and J. E. Roberts, "Local quasiequivalence and adiabatic vacuum states," Comm. Math. Phys., vol. 134, pp. 29-63, Nov 1990.

[6] I. Gelfand and V. N., Generalized Functions - Vol 4: Applications of Harmonic Analysis. Academic Press, 1964.

[7] C. Bär, N. Ginoux, and F. Pfäffle, Wave Equations on Lorentzian Manifolds and Quantization (ESI Lectures in Mathematics and Physics). American Mathematical Society, 32007.

[8] A. N. Bernal and M. Sanchez, "Smoothness of time functions and the metric splitting of globally hyperbolic spacetimes," Comm. Math. Phys., vol. 257, pp. 43-50, May 2005.

[9] P. Günther, Huygens' Principle and Hyperbolic Equations (Perspectives in Mathematics). Academic Pr, 1988.

[10] J. Dimock, "Algebras of local observables on a manifold," Comm. Math. Phys., vol. 77, pp. 219-228, Oct. 1980.

[11] J. Dimock, "Quantized electromagnetic field on a manifold," Rev. Math. Phys., vol. 04, pp. 223-233, Jun 1992.

[12] H. L. Cycon, R. G. Froese, W. Kirsch, and B. Simon, Schrödinger Operators: With Applications to Quantum Mechanics and Global Geometry (Theoretical and Mathematical Physics). Springer, corrected ed., 12008.

[13] K. Maurin, "Methods of hilbert spaces," Bull. Amer. Math. Soc., vol. 78 (2), no. 9904, pp. 15-20, 1972.

[14] K. Maurin, General eigenfunction expansions and unitary representations of topological groups. PaĹ stwowe Wydawn. Naukowe, 1968.

[15] G. E. F. Thomas, "Integration of functions with values in locally convex suslin spaces," Trans. Amer. Math. Soc, vol. 212, pp. 61-81, 1975.

[16] H. Herold, Differentialgleichungen im Komplexen (Studia mathematica : Skript) (German Edition). Vandenhoeck und Ruprecht, 1975.

[17] J. Dieudonne, Treatise on Analysis Volume II. Academic Press, 1976.

[18] G. McCabe, "The structure and interpretation of cosmology." Phil. Sci. Arch., December 2003.

[19] K. V. A. V. Petrov, A., "Classification of gravitational fields of general form by motion groups," Izvestiia Visshikh Uchebnikh Zavedeniy. Matematika, vol. 6(13), pp. 118$130,59$.

[20] H. Stephani, D. Kramer, M. MacCallum, C. Hoenselaers, and E. Herlt, Exact Solutions of Einstein's Field Equations (Cambridge Monographs on Mathematical Physics). Cambridge University Press, 2 ed., 52003.

[21] R. Camporesi, "Harmonic analysis and propagators on homogeneous spaces," Phys.Rept., vol. 196, pp. 1-134, 1990.

[22] S. Helgason, Differential Geometry, Lie Groups, and Symmetric Spaces, Volume 80 (Pure and Applied Mathematics). Academic Press, 11979.

[23] G. B. Folland, Course in Abstract Harmonic Analysis (Studies in Advanced Mathe- 
matics). CRC-Press, 1 ed., 21995.

[24] I. Gelfand and G. Shilov, Generalized Functions: Fundamental and generalized function, by IM Gel fand, and GE Shilov, vol. 2. Academic Press.

[25] E. Hebey, Nonlinear analysis on manifolds: Sobolev spaces and inequalities (Courant lecture notes in mathematics). Courant Institute of Mathematical Sciences, New York University, 1999.

[26] M. Taylor, Pseudodifferential Operators and Nonlinear Partial Differential Equations. Birkhäuser, Boston, 1991.

[27] M. Haase, The Functional Calculus for Sectorial Operators (Operator Theory: Advances and Applications). Birkhuser, 2006. 